\title{
Synthesis of colloidal nanomaterials through femtosecond laser ablation
}

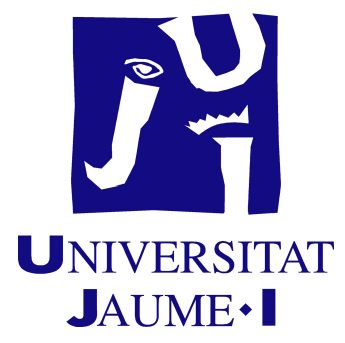

Rafael Omar Torres Mendieta

Escuela Superior de Tecnología y Ciencias Experimentales Universitat Jaume I.

A thesis submitted for the degree of

Doctor in Science

Supervisors:

Dr. Jesús Lancis Sáez

Dr. Gladys Mínguez Vega

June 2016 


\section{jy \\ UNIVERSITAT \\ JAUME•}

D. Jesús Lancis Sáez, Catedrático de la Universidad Jaume I de Castellón del área de conocimiento de Óptica, y Dña. Gladys Mínguez Vega, Profesora Titular de Óptica de la Universidad Jaume I de Castellón,

\section{CERTIFICAN:}

Que la presente memoria "SYNTHESIS OF COLOIDAL NANOMATERIALS THROUGH FEMTOSECOND LASER ABLATION", resume el trabajo realizado, bajo su dirección, por D. Rafael Omar Torres Mendieta y constituye su Tesis para optar al Grado de Doctor.

Para que así conste, en cumplimiento de la legislación vigente, firman el presente certificado en Castellón, a veinticuatro de junio del dos mil dieciséis. 
This thesis is dedicated to

my parents, my sister, and my beloveds

for always believe in me, no matter what. 
¿Qué les queda por probar a los jóvenes en este mundo de paciencia y asco? ¿sólo grafitti? ¿rock? ¿escepticismo? también les queda no decir amén no dejar que les maten el amor recuperar el habla y la utopía ser jóvenes sin prisa y con memoria situarse en una historia que es la suya no convertirse en viejos prematuros. ¿qué les queda por probar a los jóvenes en este mundo de rutina y ruina? ¿cocaína? ¿cerveza? ¿barras bravas? les queda respirar / abrir los ojos descubrir las raíces del horror inventar paz así sea a ponchazos entenderse con la naturaleza y con la lluvia y los relámpagos y con el sentimiento y con la muerte esa loca de atar y desatar.

¿qué les queda por probar a los jóvenes en este mundo de consumo y humo? ¿vértigo? ¿asaltos? ¿discotecas? también les queda discutir con dios tanto si existe como si no existe tender manos que ayudan / abrir puertas entre el corazón propio y el ajeno / sobre todo les queda hacer futuro a pesar de los ruines de pasado y los sabios granujas del presente.

Mario Benedetti 


\section{Acknowledgements}

I want to thank my supervisors Jesús Lancis Sáez and Gladys Mínguez Vega for teach and guide me in developing this thesis, besides giving me all time support and trust.

I also want to thank my research group partners and ex-partners for supporting me and contribute directly or indirectly to the elaboration of this thesis work through their advise, reference and friendship.

A special thank to my parents Marina Mendieta Saavedra and Rafael Torres Torres, for all their love, for always believe in me, for their patience and unconditional support.

To my sister Laila Anaid Torres Mendieta, to my life partner Dominika Bucová, to my best friend Manuel Alejandro Lugo, and all my dear friends who have always supported me in good times and bad times, that have always believed in me and have taught me the value of friendship.

To the University Jaume I for give me the opportunity to use their facilities, support of their workers and the support provided by the members of the SCIC of the Universitat Jaume I.

And finally I want to thank the Generalitat Valenciana council for the support from the Santiago Grisolia scholarship GRISOLIA/2013/015, that made possible that I have started to perform the research activity that eventually served as the basic point for the present thesis work. 


\section{Contents}

1 Introduction $\quad 1$

1.1 Laser . . . . . . . . . . . . . . . . . . . . . . . . 3

1.2 Interaction between laser radiation and matter . . . . . . . . . . . . 4

1.3 Nanostructures growth . . . . . . . . . . . . . 8

1.3.1 Bottom-up techniques to growth nanostructures . . . . . . . 8

1.3.2 Top-down techniques to growth nanostructures . . . . . . . . 10

1.4 Pulsed Laser Ablation in Liquids as a nanomaterial synthesis approach. 10

1.4.1 The impact of PLAL in the scientific society. . . . . . . . . . . 12

1.5 Motivation for the present work . . . . . . . . . . . . . . 14

2 Theoretical Background $\quad 18$

2.1 Pulsed Laser Ablation in Liquids . . . . . . . . . . . . . . . . 19

2.1.1 Physical parameters ................ . . 24

2.1.2 Chemical parameters . . . . . . . . . . . . . . 26

2.2 Post-irradiation process to control nanometerials characteristics after PLAL . . . . . . . . . . . . . . . . . . 28

3 Characterization techniques $\quad 34$

3.1 X-ray and electron-based techniques . . . . . . . . . . . . 35

3.2 Optical techniques . . . . . . . . . . . . . . . . . . 40

3.3 Thermal techniques . . . . . . . . . . . . . . . . . . 48

4 Setup Details $\quad 51$

4.1 PLAL and post-irradiation setups . . . . . . . . . . . . . 51

4.2 Focusing system . . . . . . . . . . . . . . . . . . . 54

5 Synthesis and characterization of gold/water nanofluids suitable for thermal applications produced by Femtosecond Laser Radiation $\quad 57$

5.1 Introduction . . . . . . . . . . . . . . . . . . . . . . 58 
5.2 Experimental Setup . . . . . . . . . . . . . . . . . . 60

5.2 .1 Material synthesis . . . . . . . . . . . . . . . . 60

5.2 .2 Preparation of samples . . . . . . . . . . . . . 61

5.3 Results and Discussion . . . . . . . . . . . . . . . 62

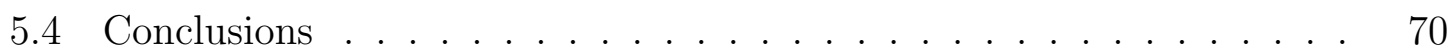

6 Fabrication of gold nanoparticles in Therminol VP-1 by laser ablation and fragmentation with fs pulses $\quad 72$

6.1 Introduction . . . . . . . . . . . . . . . . . 74

6.2 Experimental setup . . . . . . . . . . . . . . . . . 77

6.2 .1 Material synthesis . . . . . . . . . . . . . . . . . 77

6.2 .2 Preparation of nanofluids . . . . . . . . . . . . . 78

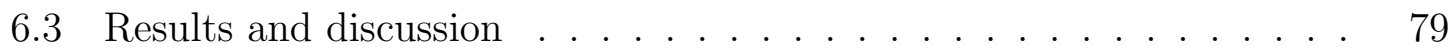

6.4 Conclusions . . . . . . . . . . . . . . . . . . . . . 90

7 Direct laser synthesis of gold nanoparticles and immobilization on $\begin{array}{ll}\text { graphene sheets } & 92\end{array}$

7.1 Introduction . . . . . . . . . . . . . . . . . . . . 93

7.2 Experimental setup . . . . . . . . . . . . . . . 96

7.2 .1 Material synthesis . . . . . . . . . . . . . . . . . . 96

7.2 .2 Preparation of samples . . . . . . . . . . . . . . . 97

7.3 Results and discussion . . . . . . . . . . . . . . . . . . 97

7.4 Conclusions . . . . . . . . . . . . . . . . . . 105

8 Conclusions 106

$\begin{array}{ll}\text { Bibliography } & 117\end{array}$ 


\section{List of Figures}

1.1 Typical laser elements: active/gain medium, pumping system and resonance cavity. . . . . . . . . . . . . . . . . . .

1.2 Macroscopic schema of the principal processes happening when light interacts with matter. . . . . . . . . . . . . . . . . . . . 4 4

1.3 Summarized interaction laser radiation matter as a function of exposure time and intensity of the source. . . . . . . . . . . . . . 6

1.4 Typical bottom-up synthesis route. . . . . . . . . . . . . . . . 9

1.5 Number of research publications and citations per year for scientific work where PLAL was used. . . . . . . . . . . . . . . . . .

1.6 world map pointing out the most proactive scientific groups working with PLAL and its respective publication record. . . . . . . . . . . . 14

2.1 Schematic draw of the interactions happening in PLAL, (I) laser-liquid interactions, (II) laser-target interactions, (III) target-liquid interactions, (IV) products-target interaction, (V) laser-products interaction, (VI) liquid-products interaction. . . . . . . . . . . . . . . . . . . . 19

2.2 PLAL experimental setup. . . . . . . . . . . . . . . . . . . 20

2.3 Illustration of the most probable detached energetic species. . . . . . 21

2.4 Plasma plume illustration. . . . . . . . . . . . . . . . 22

2.5 Synthesis approach considering a first stage where NPs are created by PLAL and a post-irradiation stage where the nanomaterials are postprocessed. . . . . . . . . . . . . . . . .

2.6 Melting mechanism in post-irradiation method where droplets of nanomaterial are formed due to the fast vibrational move of the electrons in the NPs, which is a consequence of the absorption of laser radiation in beam's focus area. . . . . . . . . . . . . . . . . . . . 
2.7 Coulomb explosion mechanism in post-irradiation method where small pieces of nanomaterial are formed due to a dissociation of original NPs, which is a consequence of the absorption of laser radiation in beam's focus area. . . . . . . . . . . . . . . . . .

2.8 Illustration of near-field plasma mechanism in post-irradiation method, where a plasma plume appear in the beam's focus area due to the absorption of hogh intensity laser radiation. . . . . . . . . . . .

3.1 High Resolution Transmission Electronic Microscopy Picture taken of one of our gold NPs samples. . . . . . . . . . . . . . . . . 38

3.2 Selected Area Diffraction Pattern picture taken of one of our gold NPs samples. . . . . . . . . . . . . . . . . 38

3.3 Schematic diagram of SPR in a metal nanostructure. . . . . . . . . . 42

3.4 Characteristic spectrum of gold NPs obtained during the experiments performed in this research work. . . . . . . . . . . . .

3.5 Size and size distribution graphic obtained of gold NPs produced during the experiments performed in this research work. . . . . . . . 46

4.1 Photograph of the laser used for the experiments. . . . . . . . . . . 52

4.2 Image of the real setup used in the experiments performed, where a dashed line shows the laser radiation path. . . . . . . . . 52

4.3 Path followed by the 2-D motion controlled stage. . . . . . . . . . 53

4.4 Experimental setup used to irradiating the colloids prepared by PLAL. 54

5.1 TEM micrographs of the samples before and after the thermal cycles. 63

5.2 EDX spectrum of a) PLAL-PPLI produced NPs (S1), b) commercial NPs in PBS (S3) and c) commercial NPs in citrate buffer (S5). . . .

5.3 Particle size distribution a) NPs produced by PLAL, b) commercial NPs stabilized in PBS and c) commercial NPs stabilized in citrate buffer. . . . . . . . . . . . . . . . . . .

5.4 Transmittance spectra of as-produced samples. The spectrum of pure water is also shown for reference (red dots). . . . . . . . . . . 65

5.5 Comparison of transmittance spectra of as-produced and cycled samples. 66

5.6 Extinction coefficient of NPs decoupled from the base fluid contribution. 67

5.7 Absorbed sunlight fraction as a function of the propagation distance in the nanofluid for the as-produced samples. . . . . . . . . . . . . 
6.1 EDX spectra of a) nanofluid 2, b) nanofluid 4, and c) nanofluid 5 (already published in [1]). . . . . . . . . . . . . . . 80

6.2 Biphenyl structural formula. . . . . . . . . . . . . . . . . . . 81

6.3 HRTEM micrographs of NPs (top) and SAED patterns taken of NPs (bottom). From left to right for nanofluid 2, 4 and 5. (already published in [1]).

6.4 Comparison of TEM micrograph for a) nanofluid 1, b) nanofluid 2, c) nanofluid 3 (already published in [2]), d) nanofluid 4, e) nanofluid 5 (already published in [1]). . . . . . . . . . . . . . .

6.5 Comparison of size and size distribution of samples (already published in $[2]$ and [1]). . . . . . . . . . . . . . . . . . . . 86

6.6 Comparison of mean particle sizes through the time (already published in [2] and [1]).

7.1 a) TEM micrograph of Au-NPs hooked on graphene sheets, and b) histogram of the NPs size distribution.

7.2 a) TEM micrograph of Au-NPs produced using $\mathrm{H}_{2} \mathrm{O}$ as liquid environment and a mean power of $40 \mathrm{~mW}$, b) histogram of the NPs size distribution. . . . . . . . . . . . . . .

7.3 a) TEM micrograph of Au-NPs produced using $\mathrm{H}_{2} \mathrm{O}$ as liquid environment and a mean power of $10 \mathrm{~mW}, \mathrm{~b}$ ) histogram of the NPs size distribution. . . . . . . . . . . . . . . . .

7.4 Micrograph of Au-NPs hooked in graphene sheets, a) SEM micrograph and b) zoom of the red square in figure a) showing the size of particles clearly bigger than $34 \mathrm{~nm}$.

7.5 FS Mapping Micrographs of gold NPs anchored in Graphene sheets, a) DF-STEM micrograph where the mapping was taken, b) emission of C, c) emission of $\mathrm{O}, \mathrm{d}$ ) emission of Au. e) EDX spectrum of Au-NPs immobilized in GO sample, f) HRTEM micrograph of Au-NPs/grapheneoxide showing the $d$ inter-planar spacing. . . . . . . . . . . . . . . 102

7.6 a) EDX spectrum of produced gold NPs under classical PLAL conditions, b) HRTEM micrograph of Au-NPs showing the $d$ inter-planar spacing

7.7 XRD patterns of graphene-oxide decorated with ligand-free gold NPs (Au-NPs/GO), reduced graphene-oxide by hydrazine method (rGO) and graphene-oxide obtained by the Hummers method (GO) . . . . . . 104 


\section{Acronyms List}

LASER: Light Amplification by Stimulated Emission of Radiation

ASE: Amplified Spontaneous Emission

UV: Ultra Violet

NPS: Nanoparticles

MNPS: Metal Nanoparticles

CVD: Chemical Vapor Deposition

MEMS: Micro-electromechanical Systems

CCD: Charge-Coupled Devices

PE: Plasma Etching

PLAL: Pulsed Laser Ablation in Liquids

PPLI: Post Processing stage based on Laser Irradiation

SPR: Surface Plasmon Resonance

ICP-MS: Inductively Coupled Plasma Mass Spectrometry

TEM: Transmission Electron Microscopy

EDX: Energy Dispersive X-ray Spectroscopy

HRTEM: High Resolution Transmission Electronic Microscopy

SAED: Selected Area Electronic Diffraction

XPS: X-ray Photoelectron Spectroscopy

SEM: Scanning Electronic Microscopy

HAADF-STEM: High-Angle Annular Dark-Field Scanning Transmission Electronic Microscopy

FS Mapping: Fast Mapping

DLS: Dynamic Light Scattering 
LDV: Laser Doppler Velocimetry

FCC: Face Centered Cubic Crystalline Structure

JCPDS: Joint Committee on Powder Diffraction Standards

PV: Photo-Voltaic cells

SPC: Solar Power Concentrators

$\mathrm{Cu}$ : Copper

C: Carbon

Au: Gold

Na: Sodium

Fe: Iron

Cr: Chromium

K: Potassium

Br: Bromide

GO: Graphene Oxide

rGO: Reduced Graphene Oxide

PBS: Phosphate Buffered Saline

DLVO: Derjaguin-Landau-Verwey-Overbeek

FWHM: Full Width Half Maximum 


\section{Chapter 1}

\section{Introduction}

Trough the human history there have been several advantages in science and technology that have changed the way in that human beings understand the reality, but maybe one of the most important is the manipulation and understanding of light. For centuries, our society has been just an spectator of its effects, mainly making the nature visible and giving us in this way the opportunity to interact with it. But it was not until 1015 A.C. when Ibn Al Haytham wrote the first rigorous book recorded [3], where the phenomenon that happens between light and matter is approached through a language that the whole world could understand, the mathematics, several times named as the language of the nature [4-6]. Since then, there have been several persons that have tried to build a little bit more the theory that is devoted to understand the nature of light and to lead us one step forward to create a better future with its help. Among the most important discoveries that take us to this thesis work, we should mention the wave nature of light proposed in 1818 by Fresnel [7], which has its basis on Thomas Young's wave theory of light, lately in 1865 James Clerk Maxwell unified the observations made by Carl Friedrich Gauss, Michael Faraday, Heinrich Lenz and André-Marie Ampère in a single theory recognized by the electromagnetic behavior of light [8]. In 1905 Albert Einstein established the fundamentals of the duality waveparticle, a new way to understand the propagation of light which is based on the idea of a discrete nature of energy proposed by Max Plank in his radiation theory [9]. From then, the theory of light has had a rapid growth giving birth to a concept which have been accepted as the definitive propagation of light, the photon and its waveparticle nature [10]. The photon is recognized as a quasi-particle or a particle that carries all of the electromagnetic radiation forms, it's mass is zero and travels trough the vacuum at a constant velocity $c(299792458 \mathrm{~m} / \mathrm{s})$ and presents particle and wave properties, the energy associated to the photon is given by the Equation 1.1, where 
$h$ represents Planck's constant, $c$ represents the light's velocity, $\lambda$ wavelength and $v$ wave frequency.

$$
E=\frac{h c}{\lambda}=h v
$$

With the definitive understanding of the light propagation nature, the technological advantages started to appear until finally in 1960 Theodor Maiman built the instrument which provided to the society the highest control over the light [11], the LASER (the acronym comes from Light Amplification by Stimulated Emission of Radiation). The laser has meant a real revolution in the understanding of interaction between light and matter, but not just in the sense that using the laser we can transmit energy to the objects or produce different kind of responses from the matter. Considering that a conventional laser produces spatial and temporal coherent light, it has been possible to proof in many ways that the interaction between light and matter occurs due to an change of energy, mainly absorption or reflection of photons with a specific amount of energy by electrons orbiting in a specific energy level belonging to atoms that form the matter. In this context, the specific energy levels in the atoms are not just determined by the number of electrons and its interaction with the atom's nucleus, it is also determined by the interaction with other atoms in its proximity and their spatial position with respect to the system that are part of. The basis on this description lies on the Bohr's atomic model and its postulates [12]. This theory wasn't built by the use of the laser, but the simplicity of the postulates that enunciate the existence of allowed and forbidden energetic levels and the possibility for absorb and emit energy between allowed levels, in combination with the use of lasers have had tremendous implications. From the discovery of nonlinear optical effects in materials with a special atomic order [13] to the imaging of carcinogenic cells in the human body through the exploitation of quantum effects produced in nanoscopic materials by its interaction with light [14-16]. Moreover, the interaction between sources of light like lasers and matter is not limited to the observation of optical phenomena, the change of energy can also promote the manipulation and alterations in the matter [17-19]. Nevertheless, not any kind of laser source produces the same kind of alterations and level of manipulation. Among the laser sources, we can divide them in two big groups, the continuum wave laser sources and pulsed laser sources. 


\subsection{Laser}

A traditional laser source is composed by an active/gain medium with multilevel system for reaching the condition of population inversion, which is located as it is depicted in Figure 1.1, between a pair of parallel and highly reflecting mirrors with one of them presenting a partial transmission used to make light oscillate between them and amplify in this way the radiation coming from the gain medium, and a pumping mechanism used to powering the active medium.

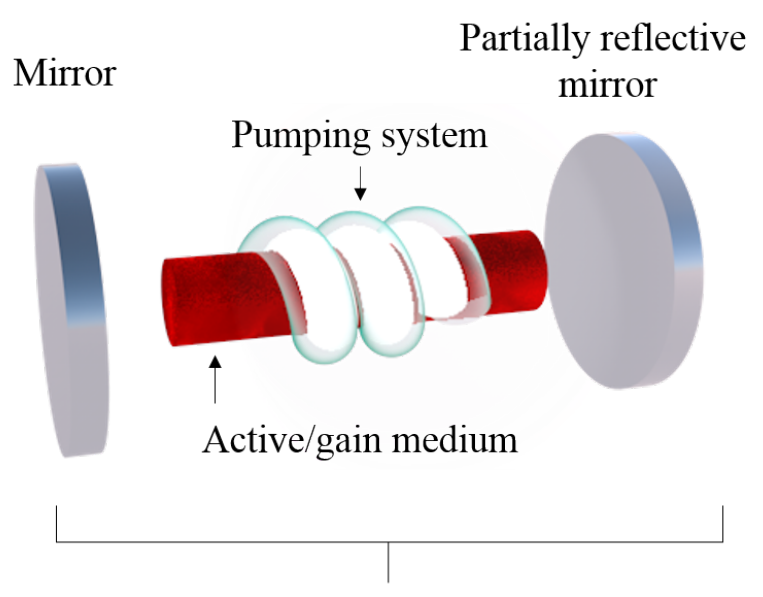

Resonance cavity

Figure 1.1: Typical laser elements: active/gain medium, pumping system and resonance cavity.

The principal difference of continuum wave laser and pulsed lasers is that this kind of laser sources emit radiation in a continuum way at a constant power. For doing it, it's not necessary further manipulation of the radiation. In this way, only photons coming from stimulated emission generated in the resonance cavity will emerge from the laser source in a temporally and spatially coherent way.

In the case of pulsed laser sources, there is an extra characteristic that differentiate them from continuum wave lasers, the production of the pulses. There are several ways to produce pulses, the easiest way is to control the pumping system, there are some lasers that when they are pumped in a non-continuum way emit train pulses with a typical temporal length of milliseconds or microseconds [20-22]. This emission way is a direct consequence of the interaction between the active medium and the pumping system, nevertheless, the pumping system limits the temporal length of the pulses. To reach shorter temporal lengths, two main techniques are commonly used, Q-switching and mode-locking. Essentially Q-switching is based on the accumulation 
of energy that comes from the pumping and its further liberation in a single shot. The idea is to amplify the spontaneous initial radiation by increasing the photons in the resonance cavity promoting the emission of accumulated energy in a form of an intense and short pulse. This is achieved by preventing the escape of radiation from the resonance cavity and preventing the input of pumping to promote the population inversion with the initial radiation until the system reaches the ideal conditions to produce laser radiation. The typical temporal range of these pulses is located in the region of nanoseconds $\left(10^{-9} s\right)[23,24]$. Mode-locking on the other hand, can reach the range of femtoseconds $\left(10^{-15} s\right)$. The idea behind this technique is based on the matching of wave modes inside the resonance cavity. This is achieved by introducing a perturbation on the longitudinal mode's phase to promote the temporal superposition of wave longitudinal modes inside the resonance cavity, constructive superposition results in very narrow light pulses with a high concentration of photons.

\subsection{Interaction between laser radiation and matter}

Traditionally, from a macroscopic point of view the matter undergoes different processes when is exposed to a light source. In Figure 1.2, the principal processes are depicted: reflection and refraction, absorption and scattering.

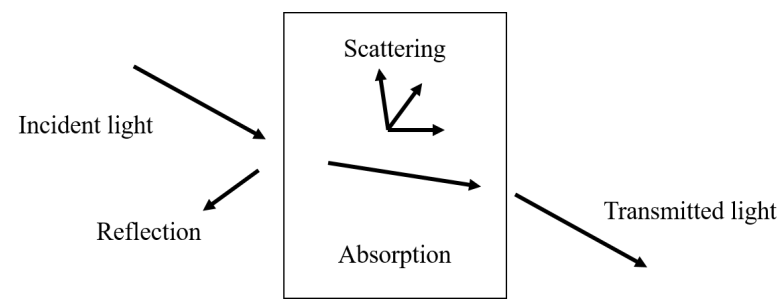

Figure 1.2: Macroscopic schema of the principal processes happening when light interacts with matter.

All of these processes result from the interaction between an oscillating electromagnetic field with charged particles that form a system. The response of the charged particles depends on the nature of the system of which they are part, and a collective response is expressed as the above mentioned processes.

Scattering for example, is the result of the deviation of the straight path of electromagnetic waves due to its collision with the charged particles forming a system. Reflection is the result of electromagnetic radiation that is either absorbed and subsequently emitted backwards or scattered in the same direction. Reflection is the result of a phase velocity change in an electromagnetic wave but conserving the frequency, 
resulting from the promotion of a temporary delay due to absorption or collisions. Absorption, as it was described above is the way in which matter takes the energy from an incoming energetic source and transforms it into internal energy that eventually can be used for further purposes.

However, even when all of the as-mentioned processes seems to be different, from an atomic point of view can be reduced to exchanges of energy between atoms forming a system and an incoming energetic source. From all of these processes, when a laser source is used to irradiate matter, the main process that leads to a chain of events that may modify the matter is the absorption. Depending on the intensity of the laser, this process will lead to different further interaction phenomenons like photochemical effects, thermal effects, photo-ablation, ablation induced by plasma and photo-disruption.

Photo-chemical effects, as it is depicted in Figure 1.3 can be promoted by every kind of laser source, these effects occur when a material is exposed to low intensity laser radiation $\left(1 \mathrm{~W} / \mathrm{cm}^{2}\right)$ for long periods of time. During the interaction time, the material's system can acquire enough energy to reach ideal conditions to promote chemical reactions in it. Probably the most illustrative example of the exploitation of this phenomenon is photo-dynamic therapy commonly applied in the fight against cancer. For this application, functionalized chromophores are injected into the body through the bloodstream, and after some time the most of the chromophores are eliminated from the human body, just few of them remain in the vicinity of carcinogenic cells due to the functionalization. Once the population of chromophores is just concentrated around the targets, the tissue is irradiated with a laser source and the chromophore triggers a series of chemical reactions that help to kill the carcinogenic cell [25].

Thermal effects are associated to an increase in the material's temperature due to the absorption of radiation. Therefore, long periods of exposure time are also required, nevertheless in this case, the use of continuum wave lasers or pulsed lasers cause different effects. The thermal effects happens because the laser irradiation of any kind of atom can produce the absorption of its photons, when this happens, the electrons jump to a higher energetic level producing in this way an excited state of the atom, this kind of state is not completely stable in the nature for very prolonged periods of time, then the natural tendency of the systems is go down to their basal state. To do this, there are many ways that occur at short periods of time beginning from the most easy one, emitting the excess of energy as a photon, as a vibrational move, as a rotational move, as a translational move, as a radioactive decay, etc [26]. 


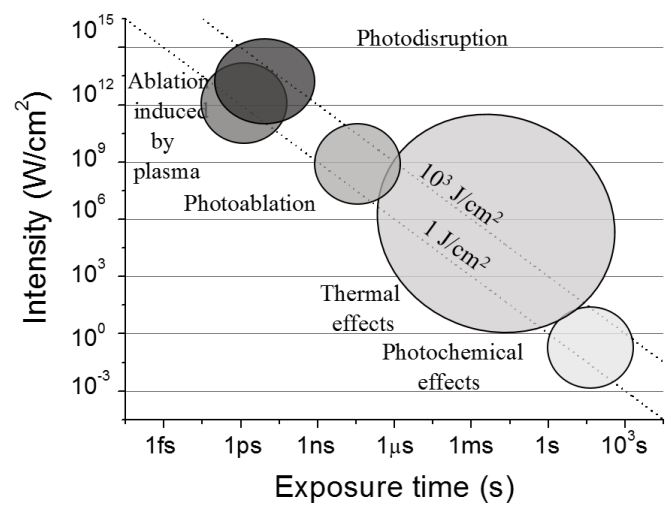

Figure 1.3: Summarized interaction laser radiation matter as a function of exposure time and intensity of the source.

At this point it's clear to notice that if the material is exposed for a prolonged period of time to the absorption process, not all of the electrons exposed to the radiation are going to be able to reach an stable energy level and consequently are not going to be able to rid of energy excess by emitting a photon and instead of that the most probable way is the vibration. If this happens in an atomic system, when the electrons vibrate, they transfer that energy to neighbor electrons producing in this way the spread of the energy, but also structural rearrangements can be promoted in the system leading to phenomenons that can be exploited for applications, such as metal melting [27], vaporization of materials [28], plasma generation [29], thermal treatment to materials [30], among others.

Photo-ablation is a process in which material is detached from a macroscopic solid. The fundamental characteristic of this effect lies on the energy per photon used, in particular the name "photo-ablation" is traditionally used just for the process in which ablation is promoted by Ultra Violet (UV) laser sources, i. e. laser sources with a wavelength centered in the spectral region from 150 to $400 \mathrm{~nm}$. Photons in this spectral region have enough energy to excite molecules in solid systems until reach their dissociation threshold. To be more precise, UV photons have the required energy to be absorbed by electrons in molecular bonds, when the electrons absorb the amount of UV photon's energy, they tend to get excitation levels that exceed the energy of the most of molecular bonds. The excess of energy tends to dissipate through thermal effects. The typical range of intensities at which this effect happens is $10^{7}-10^{10} \mathrm{~W} / \mathrm{cm}^{2}$. The most common application for this process is refractive eye surgery, where the wavelength ranges used don't generates heat in the treated 
tissues, although their power is such that ablates the top layer of tissue in which it is applied [31].

At this point is important to notice that, the energy per area (fluence) achieved by the laser sources strongly depends on the pulse duration and the central wavelength of the source. In this context, the fluence can reach high values in short periods of time (the pulse duration time length). When a high concentration of photons is interacting with matter in a reduced time and space the response of matter includes but is not limited to thermal effects. When the intensity starts to exceed the threshold of thermal effects which is $10^{6} \mathrm{~W} / \mathrm{cm}^{2}$ for the normally used materials, processes like ablation (photo-ablation, ablation induced by plasma) or photo-disruption start to be present.

Ablation induced by plasma for instance, is a process that achieve its biggest efficiency when pulsed laser sources are used, due to these sources reach an energy range of $10^{11}-10^{13} \mathrm{~W} / \mathrm{cm}^{2}$ in which photo-ionization occur at a high rate. In this effect, incoming photons are absorbed by electrons that are part of the irradiated system, they are pulled out of the system by the influence of the incoming electromagnetic field associated to laser radiation, the electrons form a cloud above the irradiated material and continue absorbing energy from the incoming photons and acquire kinetic energy, eventually they transfer their energy to the recently created ions and another energetic species, and the phenomenon leads to the generation of a plasma. When the energy is not so high, electromagnetic interaction are induced by the plasma, promoting the detachment of material by breaking molecular and ionic bonds. This process is known as Coulombian explosion. When the energy is so high, the excess of energy is transferred to the solid material through thermal effects at a high velocity leading to extremely elevated pressure conditions in the irradiated area allowing the detachment of more material in an explosive way. This process is commonly known as phase explosion. The exploitation of this phenomena have carried great applications widely used in the industry and science research, such as, cutting [32], drilling [33], welding [34], annealing [35], and the most important for the present research work, the selective fabrication of nanostructures [36]. In this context, the use of laser sources to induce nanostructures have rapidly grown in the recent years, envisaging its strongest application in the fabrication of nanoparticles (NPs).

Photo-disruption in the other hand, is a process which follows ablation induced by plasma when the intensities used are above $10^{11} \mathrm{~W} / \mathrm{cm}^{2}$. In this process, besides the aforementioned phenomena occurring during ablation, a mechanical secondary effect is promoted. The release of energy after the detachment of material induce shock 
waves leading to fissuration, cavitation, formation of defects, etc. The process allows the detachment of more material during ablation or it could also be used to induce defects in the materials. The most common application for this effect is intra-ocular surgery, it is normally used to asses a high precision in tissue cutting [37].

\subsection{Nanostructures growth}

Nanostructure growth have gained a lot of attention since the idea of its exploitation was conceived in 1959 by Richard Philip Feynman, promising the next biggest technological revolution. During the 50's decade the idea of directly manipulating the atoms in the field of chemical synthesis was not completely accepted, despite that as early as in 1857 Michael Faraday has already created colloidal gold suspensions that are still stable until our time. Nevertheless, the most daring ideas always find their niche. In 1951, John Turkevich have reported the first standard method to prepare metal colloids (Gold particles with an average size of $20 \mathrm{~nm}$ prepared by the reduction of $\mathrm{AuCl}_{4}^{-}$with sodium citrate), but it was not until 1970 when supported by G. Kim proposed a possible explanation of nanoparticle formation based on nucleation and growth [38]. The development of a reproducible technique to grow nanostructures facilitated that laboratories worldwide started to study its special characteristics to further take advantage of them. Nowadays, the global industry demands the fabrication and use of every day, more and better nanostructures to enhance macroscopic system's properties. To face this reality, several groups have developed a lot of nanostructure fabrication techniques that can be summarized in two big groups, Bottom-up and top-down techniques.

\subsubsection{Bottom-up techniques to growth nanostructures}

Historically, the most popular methods to produce nanostructures have been strategies based on the arrangement and assembly of atoms and molecules to build structures. The idea is to have a control over short-range attraction forces such as Van der Waals, electrostatic, and a variety of inter-atomic or intermolecular forces, by means of two simple stages, promotion of chemical reactions and limitation of them. The simplest example to have a picture on mind is the following: As it is depicted in Figure 1.4, a compound which contains ions of a nanoparticle desired material normally known as precursor agent is mixed with a compound commonly called reductor that reacts with the precursor, the mixture starts to react under special conditions. During the reaction the ions of the NPs desired material are going to be liberated and 
reduced to a ground state, the reduced ions are going to start to stick to each other in a process known as nucleation. This process can continue for a prolonged period of time forming bigger and bigger clusters until the reaction gets stopped abruptly by changing the reaction conditions or by the introduction of another element that would block the reaction. Nevertheless, is important to notice that this kind of protocol is not usually as ideal as it was described in the example. In the reality, not all of the ions are equally reduced at the same period of time. When the reaction process is stopped, the chemical processes do not stop in an homogeneous way in all of the mixture, which leads to the fabrication of materials that present chemical cross effects. It is not easy to find the special conditions to produce reduction of any kind of material, and finally, the chemical waste produced during this protocol is not always low $[39,40]$.

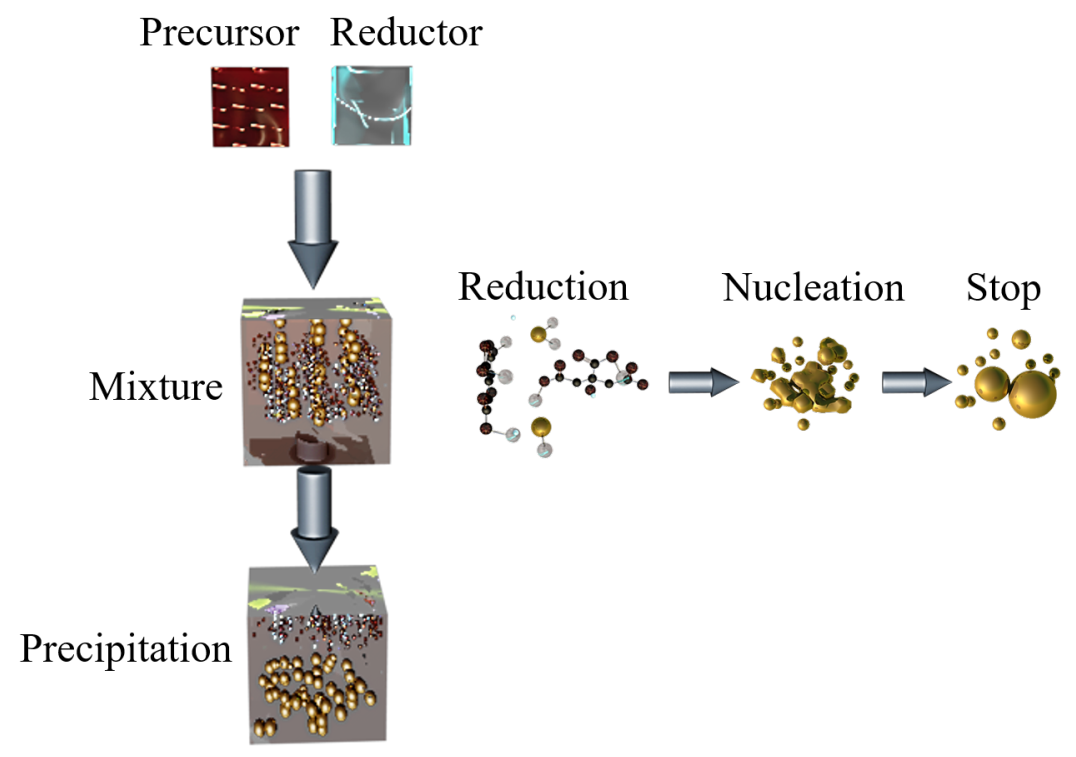

Figure 1.4: Typical bottom-up synthesis route.

However, there are several synthesis routes that follow this principles, mainly because they facilitate the production of large quantities of material without requiring so expensive material and promise a high control over nanostructure growth. Among the most important we can mention sol-gel processing, laser pyrolysis, chemical vapor deposition (CVD), plasma or flame spraying synthesis, atomic or molecular condensation, etc.

The ability to build things from the bottom to up promise its future incorporation to the industry, but nowadays is still limited by the present technology resulting in the impossibility to produce complex structures. Just until we have completely 
improved the bottom-up synthesis approach, we are not going to be able to fully achieve materials that can be the base bricks for building the new technology that Richard Feynman talked about.

\subsubsection{Top-down techniques to growth nanostructures}

In the other hand, the story of the top-down approach goes along with the industry development, the first useful synthesis was crated to solve micro-machining problems, such as the manufacturing of micro-electromechanical systems (MEMS) [41]. Nevertheless, its implementation in the industry showed its biggest weakness, this kind of approach is not suitable for its implementation in a very large scale, mainly because technological and economical limitations. But over the time, the discovery and understand of this weak point brought also its implementation in different fields where the manufacture of complicated nanometric structures was required in not large scales. Compared with bottom-up fabrication routes, top-down techniques present an advantage that is catching the attention of more and more research groups, the possibility to manipulate matter in a friendly environmental way. In top-down approach, all begins from a macroscopic piece of material. The central idea is to, step by step remove nanometer size pieces from it by breaking bonds between molecules when a source of energy is applied, in order to build a complex new nanometer structured system producing low pollution. Some of the most well-known techniques that follow this principle are photo-lithography [42], electron beam lithography [43], anodization [44], ion- and plasma etching (PE) [45], sputtering [46], laser ablation [47], among others. The most of these ways of nanometrial production are suitable for produce complex structures of almost any kind of material. But from now on, we are going to focus our attention in an emerging technique that promises to change the way in that NPs are built, Pulsed Laser Ablation in Liquids (PLAL) a technique that combines a top-down and bottom-up mechanisms.

\subsection{Pulsed Laser Ablation in Liquids as a nanoma- terial synthesis approach.}

Arising from its inherent interdisciplinary, nanotechnology is being recognized as a technological revolution that will affect many of the aspects of human society, presenting answers to solve some of the grand challenges that face our society as energy production, climate change and health [48-50]. But to build an environmentally 
sustainable world, a "green" method to produce NPs and reduce the waste is a necessity. For applications where a low or moderate production rate is required, PLAL has released as a promising alternative to chemistry methods, thanks to the nearly unlimited nanomaterial products that can be synthesized, the simplicity of the procedure and the absence of unnecessary adducts and byproducts [51]. PLAL is based on the detachment of material located at the surface of a solid target, which is immersed in a liquid environment by means of its interaction with ultra-intense laser radiation. The detached material is commonly extracted in the form of an ablation plume, which contains atoms, ions and clusters. NPs are formed from the ejected material and they are collected in the liquid forming stable colloids. The nature of the synthesis route allows the design of complex nanoscopic structures, since the concept of a liquid environment is as general as an incompressible environment filled with tiny vibrating particles of matter, linked by intermolecular bonds, and at the same time, a solid target could be any kind of solid material, PLAL permit an unimaginable number of possibilities.

\section{The main pros of using PLAL are the following:}

- Makes possible to create novel materials that don't exist in Nature.

- Offers the possibility to create nanomaterials in liquid environments where its production by conventional approaches results almost impossible.

- Absence of chemical by-products or chemical waste.

- Is a relatively low-cost synthesis route, since experimental setup and manual operation are minimal.

- Is one of the fastest, cleanest and cheapest synthesis route to produce nanomaterials dispersed in liquids with size distributions lower than 100 $\mathrm{nm}$ in gram scale.

- Finally, permits a high degree of reproducibility.

\section{And the main contras of using PLAL are the following:}

- There is a significant knowledge deficit on the basic processes happening in the formation and growth of nanostructures.

- The production rate has limitations. 
- All liquid-nanoparticle material possible combination, requires its own optimal process conditions.

- And finally, the control over the morphology is still impossible, except for few cases.

Beyond that further research is needed to understand the technique in its entirely. From a practical point of view, if you get to control very well the interactions between the liquid environment and the solid target, is feasible producing high quality nanomaterials in few minutes by the use of simple experimental setups, the adoption of this technique as starting point for the design of nanoscopic systems is increasing. The spread of this technique is not limited to its implementation on nanomedicine, sensing electronics, optics or bio-photonics, recently it has been also extended to fields like nanomaterial's conjugation [52], design of hybrid structures [53], or advanced functional material's design [54]. This has lead to an exponential increase in the publication rate of scientific and technological articles where PLAL is used at some point of the research work.

\subsubsection{The impact of PLAL in the scientific society.}

The analysis of the impact of PLAL as an efficient synthesis approach can be enlightened through a formal bibliometric review of the works published around it. For this, Science Citation Index (1990-2016) search algorithm from Web of Science is used.

From it's formal establishment in 1993 until know, the databases of literature related to NPs created by laser ablation in liquids reaches 28994 results if we consider the keywords "laser nanoparticles". Nevertheless, as it was mentioned above, PLAL is becoming an initial point or form part of the design of complex nanometric systems. Therefore, the right keywords should emerge from an optimized analysis of the abstracts of the top ten most relevant articles in the field [55-64]. Normally, in the most of the abstracts, the words used to describe nano-systems are: nanoparticle, nanocrystal, nanowire, nanotub, nanofibre, nanoshpere, nanorod, nanodot, quantum dot or quantum wire. The words used to describe laser processing are: laser ablation, laser cutting, laser welding, laser cladding, laser sintering, laser structuring, laser marking and laser soldering. Finally, the normal words used to refer to a liquid medium are: liquid, water, solvent, colloid, hydrosol, hydro-sol, alkan, solution, propanol, dispers, suspens, aqueous. To narrow the search criterion, ensuring the use of PLAL in the papers found, is mandatory to restrict the keywords due to the bast and active research work on nanoscience and its relationship with laser sources. The 
criterion then, should include the possible combination of words related to nanomaterial, laser processing, liquid environment, but exclude the words related to another popular routes to synthesis nanomaterials like gas phase, vapour deposition, vacuum, pld, surface and also exclude popular uses of laser to study nanomaterials like laser spectroscopy. After the search refinement, the number of research published works in which fabrication of nanomaterials is performed by PLAL is: 1319 .

a)

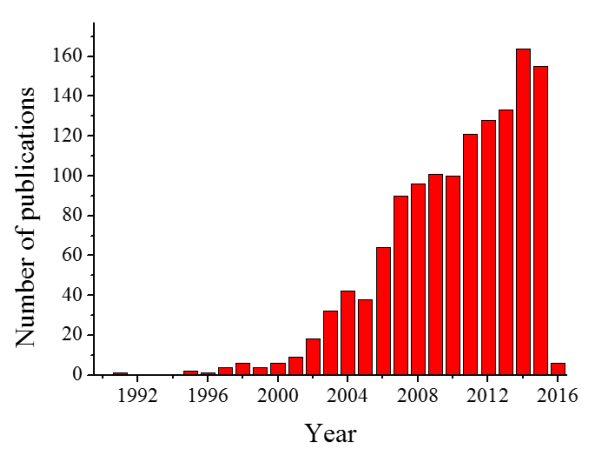

b)

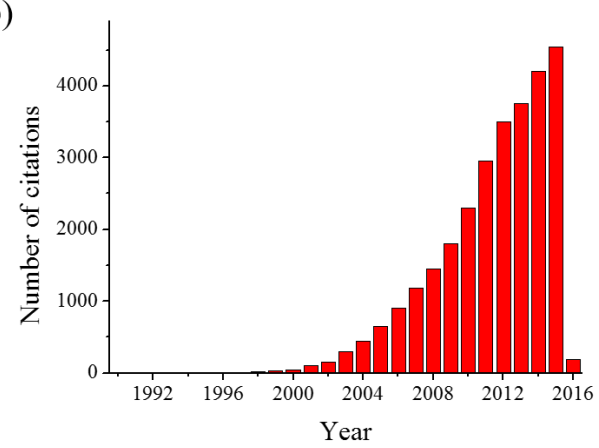

Figure 1.5: Number of research publications and citations per year for scientific work where PLAL was used.

Due to the versatility and reliability of the technique, several groups worldwide are increasing the number of high impact publications on this topic, reaching an average citation number of 100 per year, being (S. Link and M.A. El-Sayed, J. Phys. Chem, 103 8410, (1999)) the most cited article with an average citation of 126.89 per year and a total citation number of 2284. As it can be inferred from graphics depicted in Figure 1.5 that show an exponential citation and publish growth, the scientific interest on this technique is rapidly increasing.

The most proactive research groups belong to leading countries in the development of new technologies, occupying the top positions USA, Japan, People's Republic of China and Germany, with a maximum publication record of 278 and a minimum of 127. The subject areas with the highest number of contributions are physical chemistry, chemistry and materials science. In particular, materials science and chemistry are subjects with high economical incomes due to its commercial potential, and the adoption of this technique may involve its further implementation in other fields where high quality nanomaterials are required such as bio-medicine. Nonetheless, PLAL is still a young technique that requires further research effort to become popular in fields that potentially can change the way in which human beings interact with the environment. However, the intense increment in scientific activity around a young 


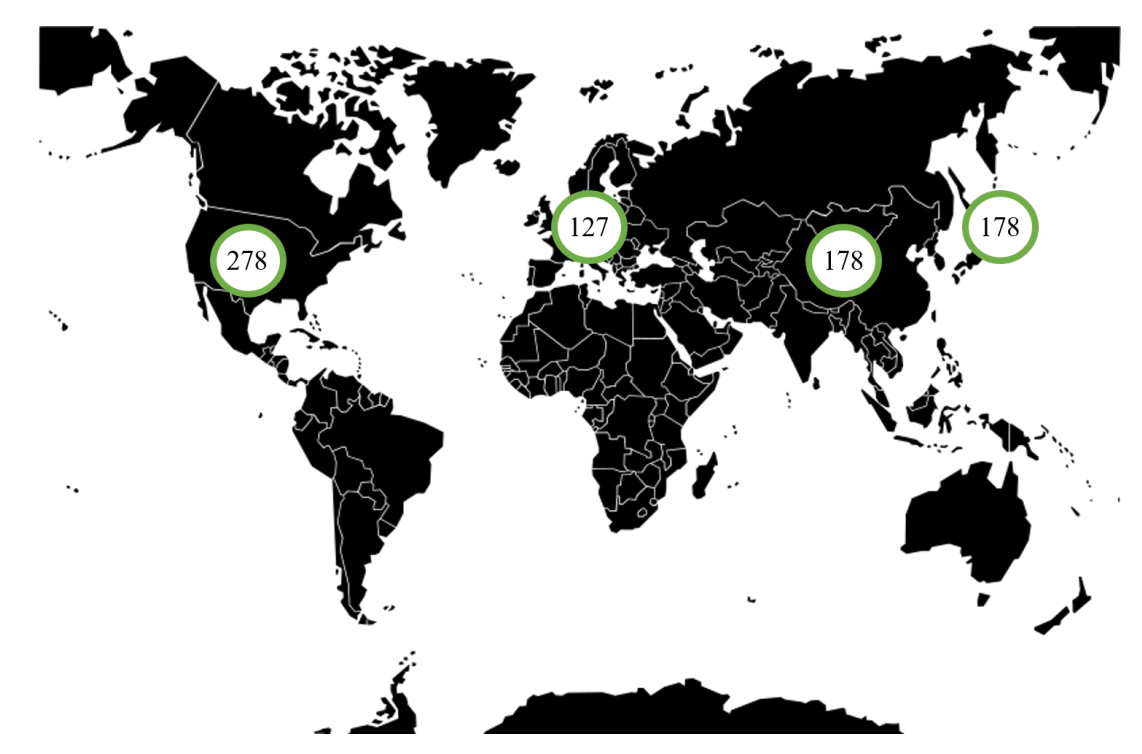

Figure 1.6: world map pointing out the most proactive scientific groups working with PLAL and its respective publication record.

nanostructure fabrication technique is an evidence of its positive impact on the current technological development and a strong reason to keep trying to discover each physical and chemical phenomenon associated with their use.

\subsection{Motivation for the present work}

Intelligent routes to synthesize highly pure, physically and chemically active NPs that can serve as building blocks to create complex systems demands not just the control over particle shape, size and size distribution, also requires the control over their surface and internal properties. It should also present a general applicability over a wide range of materials and a high reproducibility to be used in large scale nanomaterial's production. Technologically advanced applications also require the use of new nanomaterials with outstanding properties, and at the same time seeks to achieve the best results promoting a minimal negative environmental impact, during the manufacture or useful lifetime of the applications. Considering the above mentioned goals, the synthesis of nanomaterials by means of PLAL has a high potential to become a standard NPs fabrication route that can promise reliability, reproducibility and versatility.

The use of laser radiation to acquire nanostructures from a solid macroscopic material immersed in a liquid environment, provides to PLAL the unique potential to generate clean nanostructures in liquid mediums where conventional synthesis routes could hardly or not achieve. Either the physical and chemical characteristics of the 
obtained nanostructures are based on laser, solid target, and liquid environment properties. The correct combination of those properties could even lead to the creation of new materials that doesn't exist in the nature, giving the opportunity to obtain complex nanoscopic systems that may present outstanding properties.

Since PLAL is still a young synthesis approach and further research is required to understand all the mechanisms involved in the nanostructures creation, and generalize the implementation of the technique to synthesize nanomaterials from a wide range of materials in a wide range of liquid environments. The scientific activity conducted in this thesis work follows a clear objective, shed light on the understanding of the processes involved in the synthesis of materials structured at the level of the nanoscale when PLAL is used. The above is approached from an experimental perspective. Since pulsed laser radiation with pulse widths in the range of nanoseconds and picoseconds has been widely studied, it is necessary to investigate the use of pulsed laser sources with shorter pulse widths, therefore we explore the use of femtosecond pulsed laser sources in PLAL.

Moreover, considering that the range of liquid environments explored shouldn't be limited to solvents with no remarkable characteristics, we have explored the implementation of PLAL in creating nanostructures in non-conventional liquids with outstanding properties, to give rise to nanofluids and colloidal nanostructured hybrids. In this context, since the fabrication of metal nanostructures in low concentrations in heat transfer fluids has demonstrated to improve their thermal and optical properties, resulting in efficient heat transfer fluids, or volumetric sunlight absorbers, that promise to transform the solar radiation into consumable electricity having an impact on the harvesting of solar light field. We have explored the use of PLAL to solve the current issues of producing such kind of material by traditional synthesis routes, that are mostly related to particle purity problems and poor stability. In the other hand, is well known that hybrid materials composed by nanometric structures are being increasingly used in many fields, but up to now the traditional routes to produce such kind of hybrids, are based on non-environmental friendly techniques that are limited to a narrow range of materials. Since one of the greatest advantages of PLAL is the possibility to create nanostructures from an unlimited combination of solid targets and liquid mediums in a "green" way, we have explored the use of PLAL to be used as a basic technique on the production of nanometric assembles composed by graphene and metals with interesting physical and chemical features.

Aside of the main objective, the challenge of this thesis work does not lies just on the synthesis of nanomaterials, but rather on the integration of the produced nanos- 
tructures into existing materials, averting the deactivation of their unique properties due to agglomeration phenomenon or chemical contamination and offering new compounds through the methodically exploration of further modifications of PLAL. In this context, an extensive research work was performed to implement PLAL together with the use of a post fragmentation process and stabilizer agents, to obtain highly stable nanofluids and highly pure nano-hybrids composed of graphene sheets and ligand-free metal NPs.

The biggest value of this research work lies: on exploring the use of femtosecond pulsed laser sources to implement PLAL, on the production of novel high stable nanomaterials that present remarkable optical, thermal and mechanical characteristics, on expanding the databases of useful fluids or colloids, solid materials and their mixture, and finally on implement a full working PLAL synthesis station in the Universitat Jaume I for the first time.

The information in this thesis will be presented in 8 chapters. The brief highlight of these chapters is as follow:

Chapter 1 Presents an introductory text about the reason why the use of light matter interaction can lead to a high quality synthesis of complex nanostructures. The reason why lasers are suitable light sources for this, which kind of interaction between those light sources and matter are necessary to manufacture nanostructures, the creation of nanostructures by other routes are addressed, and finally a brief introduction to PLAL, the central NPs synthesis approach based on ultra short laser radiation used in this thesis work.

Chapter 2 This chapter presents the most representative details of PLAL. Aditionally, a detailed description of a further processing stage to develop a greater control over the final characteristics of nanomaterials developed by PLAL is presented.

Chapter 3 An extensive review of the nanomaterial characterization techniques used is presented.

Chapter 4 Describes each single part of the experimental details used for the experiments that resulted in this thesis work.

Chapter 5 This chapter, discusses in the detail the necessary elements that can lead to obtaining highly stable nanofluids obtained by PLAL based on gold NPs that can work as both heat transfer fluids and volumetric absorbers.

Chapter 6 This chapter, discusses in detail the synthesis of gold NPs by PLAL in an euthectic mixture of diphenil oxide and diphenil (a fluid normally used in the harvesting of solar light industry), in order to improve the fluid's thermal conductivity. 
Chapter 7 This chapter presents a discussion of the use of PLAL in the creation and subsequent anchor of metal particles on carbon-based matrices, which promises great results that can be used in applications from optoelectronics to biomedicine.

Chapter 8 Finally, this chapter present a brief summary of the research work done, the future scope of the investigation efforts, and the new direction that the work is taking. 


\section{Chapter 2}

\section{Theoretical Background}

Pulsed Laser Ablation is a method that has been widely studied under vacuum and controlled atmosphere since its development in 1960's after the invention of ruby laser. The first effect observed when a laser source was focused on a solid was the removal of some material from the surface where the laser radiation was interacting with the solid. Due to this, the phenomenon was called ablation that comes from the Latin word ablatio that means removal [65]. From that moment, the machining of almost any kind of solid material started to be exploited observing also the manufacturing of micro and nanostructures. But it was not until the nineties, when the laser ablation of samples submerged in liquid media was used for colloidal suspension preparation [66]. The main motivation for producing ablation of solids in dense mediums like liquids was the seek of an easy way to produce nanostructures that present special characteristics like oxidation. Indeed, the first work published in this direction by Patil et al. [67], was a study to synthesize a metastable form of iron oxide. The idea behind the use of ablation in a liquid environment was to promote the oxidation of iron due to the interaction between the interface liquid-solid and the laser radiation. The method became popular when Neddersen et al. [68], reported the fabrication of colloidal suspensions by laser ablation in water and organic solvents, pointing out that the method produces colloids without residual species. The possibility to produce pure NPs of almost any kind of material suspended in liquids gained the attention of several fields like chemical catalysis [69], harvesting of energy [2], bio-medicine [70], among others, due to the envisage that pure NPs would be more efficient since no byproducts are present and the particle surface is clean. With the time, it was discovered that several parameters promote processes that provide the ability to control the final quality of the manufactured structures, giving the chance to develop a high control over the particle size [71], morphology [72], functionalization [73], etc. In general, all of the processes directly involved in the manipulation of 
material changes can be summarized in interactions between the constituents in the process and the produced material, as it is depicted in Figure 2.1.

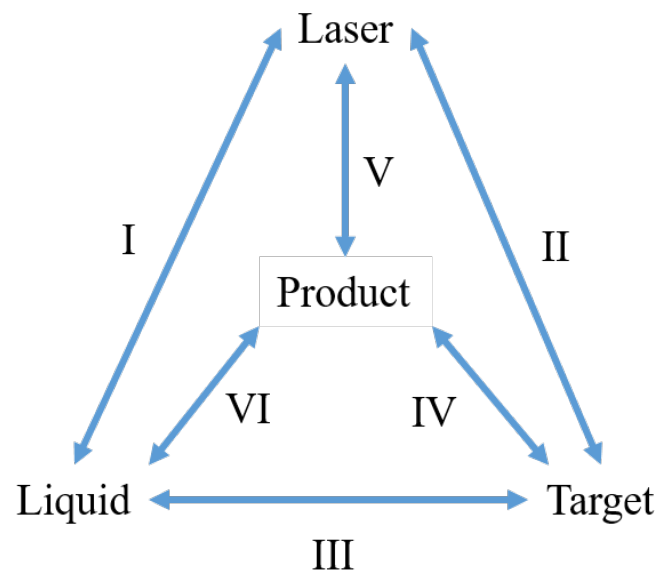

Figure 2.1: Schematic draw of the interactions happening in PLAL, (I) laserliquid interactions, (II) laser-target interactions, (III) target-liquid interactions, (IV) products-target interaction, (V) laser-products interaction, (VI) liquid-products interaction.

These interactions can be described in turn, in physical and chemical processes, the physical ones are governed by the radiation parameters and its way to interact with the solid material, the chemical processes mainly by the influence of the the solid material and liquid environment in particle creation process and its interaction with the recently created structures.

The objective of this chapter is to highlight the most important parameters that provide to this technique the opportunity to manipulate matter producing nanomaterials.

\subsection{Pulsed Laser Ablation in Liquids}

The primitive schematic draw of the experimental setup used for producing nanostructures by laser ablation in a liquid environments is depicted in Figure 2.2. Laser radiation is focused on the surface of a solid macroscopic material immersed in a liquid or colloidal ambiance. The interaction between the atoms at the material's surface, the atoms of the liquid or colloidal medium located at the liquid-solid interface and the electromagnetic field of the laser source, promotes an energy exchange in which charged particles from both solid target and liquid ambiance are affected. 


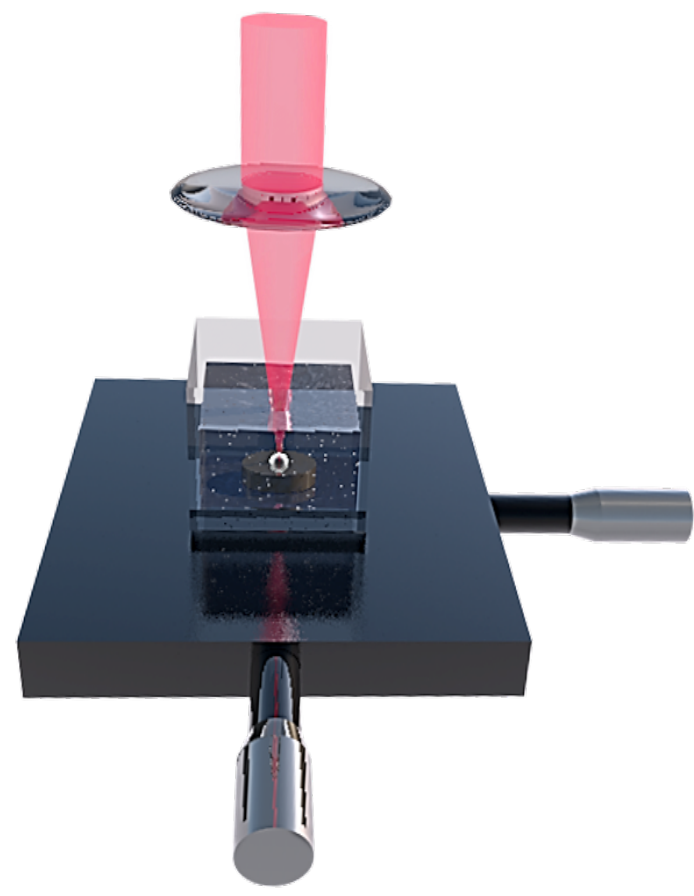

Figure 2.2: PLAL experimental setup.

To have a better understanding of the phenomenon in its entirety, is mandatory to analyse the temporal evolution of the energy exchange during the process.

$t<0$ interaction liquid-laser radiation. The first important interaction occurs between the laser radiation and the liquid environment which surrounds the solid target. Ideally, to have the best performance rate, the laser energy should be delivered just to the solid target and not to the liquid medium. Normally, the laser sources used to produce nanostructures are pulsed laser sources which carry a high density of photons in short temporal lengths, when this kind of radiation is focused the photon density increases in a very small space promoting in this way non-linear optical effects when interacting with the liquid medium such as liquid breakdown, selffocusing, among others $[74,75]$. The way to avoid this phenomena is through defocus conditions and don't reach a fluence range where non-linear effects are commonly present.

$t=0$ absorption of the laser radiation. Once we can ensure the best energy delivery into the solid target, considering non relevant the linear absorption of the liquid, the second important process is the absorption of energy by the solid target. Using the threshold fluence required for PLAL, electron-lattice thermalization and instantaneous multi-photon absorption occurs, being the last one the most probable 
one when pulse duration of the laser radiation is below $10^{-8} s[76]$. However, both processes can occur simultaneously producing thermionic emission, vaporization, boiling and melting or direct photo-ionization, respectively [77,78].

$10^{-12} s<t<10^{-10} s$ detachment of material from the solid target. Considering that instantaneous multi-photon absorption is the most probable way of light-matter interaction. The electrons in the target's surface that absorb the photons gain enough energy to leave their respective orbits leading to a mutual repulsion between atoms positively charged. The repulsion leads to an explosion into energetic electrons and ions, creating in this way a cloud of electrons with an excess of energy and atoms with a lack of electrons close to the area where the laser radiation is interacting with the solid target, as it is depicted by the Figure 2.3. Furthermore, excited electrons which doesn't leave the target's surface transfer their kinetic energy to the atom's lattice that form the solid system, resulting also in the detachment of target's material [79].
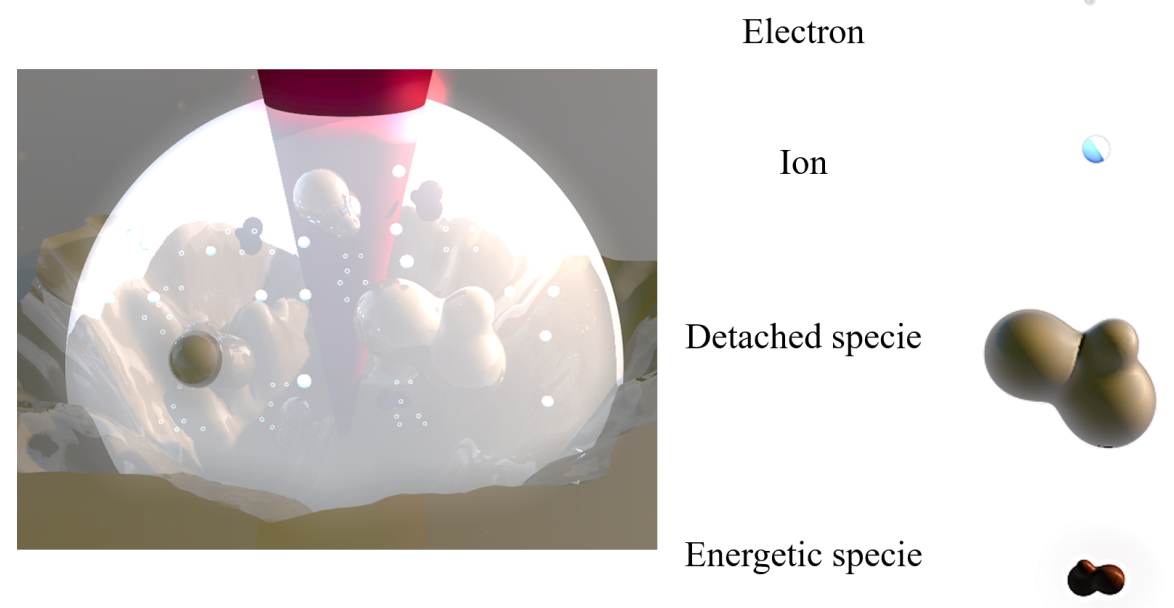

Figure 2.3: Illustration of the most probable detached energetic species.

Traditionally, the theories which describes this phenomenon are thermal processes: vaporization, boiling and explosive boiling [80]. When pulsed lasers with pulse width under $10^{-7} s$ are used, the mechanism which leads the separation of material is explosive boiling, which happens when a solid material is superheated at a high velocity, promoting in this way a decomposition of the material in vapor and liquid phase [81]. Nevertheless, when pulse widths under $10^{-12} s$ are used, the energy absorption process 
becomes extremely fast, and thermodynamic theories which consider thermal equilibrium can't describe the process properly. In this case, hot atoms, vapor and liquid drops are expelled of the material under non-equilibrium conditions. This process is called direct fragmentation [82].

When the material's ejection is taking place in both processes direct fragmentation and photo-ionization, at the same time a pressure wave propagates spherically through both solid target and liquid environment, facilitating the detachment of target's matter [83]. All of the ejected species form a cumulus containing matter that is not in thermodynamic equilibrium, the energetic species interact with atoms in their vicinity promoting the emission of photons with different energies, the whole entity is known as laser plasma plume [77], illustrated in Figure 2.4 .

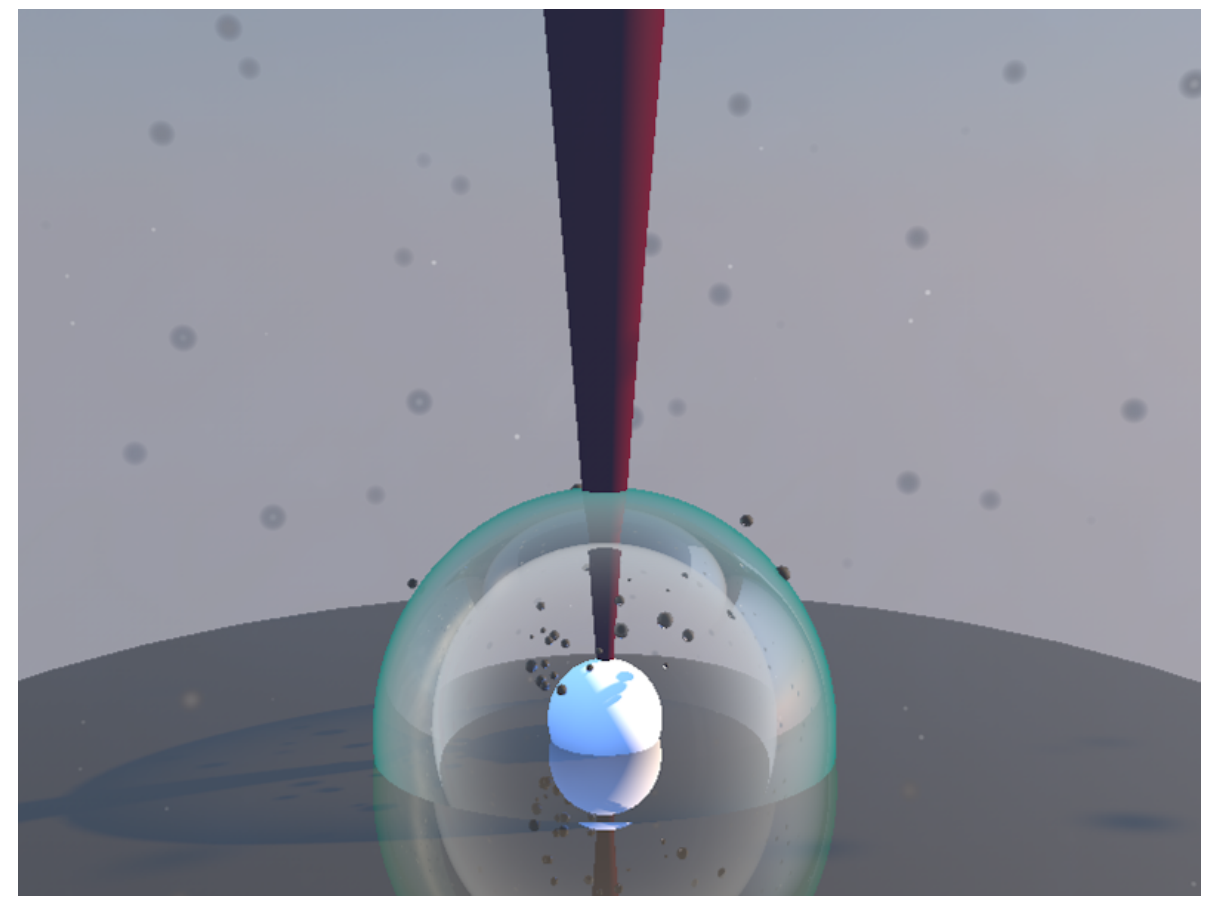

Figure 2.4: Plasma plume illustration.

$10^{-10} s<t<10^{-7} s$ plasma plume lifetime and cavitation bubble creation. The plasma plume is similar when the ablation is performed using gas or liquid environment, the difference lies on the density of the environmental medium, when a medium with higher density than the most of the gases is used the plasma plume gets confined above the ablation area keeping in direct contact with the radiated area [84]. In this way, the energetic species also interact with the target's surface promoting the exchange of energy and facilitating the extraction of material even after the pulse duration. The lifetime of laser plume starts to extinguish on the 
range of $10^{-8}-10^{-7} s$ transmitting its energy to the surrounding medium and the solid target [85]. The transmission of energy to the surrounding medium triggers the formation of a cavitation bubble, which expands to a maximum radius in the order of millimeters [86]. Its expansion causes a consumption of energy ending up to collapse.

$t>10^{-6} s$ nanomaterial growth and release of energy. During the ablation process happens, NPs formation takes place. The exact mechanism in which NPs are formed is not completely clear, but up to now it is believed that the most probable mechanism is nucleation and growth of nanometric structures that result from a mixture of the energetic material that has broken away from the target, and the solution molecules arising after its degradation, ionization and pyrolysis due to its interaction with the radiation [76]. The period of time when each of this steps takes place is not clear and further investigation is required. Nevertheless, is well known that nucleation and growth rapidly occur and formed NPs travel in cavitation bubbles [87]. Special attention should be given in future investigations to the cavitation bubble formation and its influence in NPs formation because some research groups are suggesting the hypothesis that actually NPs are formed inside the bubble during its expansion due to a mutual gradual release of energy, reaching its final form when NPs become systems with no energetic excess [88]. However, no matter the actual mechanism in which the NPs are formed, they tend to acquire spherical shape. As energetic systems coalesce to form stable systems, the simplest way to minimize the energy is an arrangement of the atomic lattice which permit reach the stability. It means that the particle is going to have a high temperature allowing the atomic free mobility, and when it comes into contact with a cooler system the atoms located at the particle surface interact with it obtaining a higher mean energy than the atoms located inside the particle, therefore the tendency of the system is to diminish the total energy, which is achieved by decreasing the number of atoms located at the surface, hence the particle acquires the minimum surface shape possible, which is the spherical one [89]. Once the particles reach the stability, minimal modification can still happen, mainly due to its interaction with energetic species that still survive.

At this point is important to notice that using this technique, NPs can be produced from almost any kind of solid material, and they can be produced in almost any kind of liquid or colloidal medium. However, due to the amount of energy used, physical and chemical phenomenons are promoted during the particle creation. Therefore, the NPs not necessarily present the same chemical composition of the solid target, and even more, a change in energy can trigger different physical and chemical phenomenons 
in different materials [90]. Hence, is mandatory to discuss each important parameter involved in NPs creation in detail.

\subsubsection{Physical parameters}

Probably the most important physical parameters that can be highly controlled are the laser parameters and the way to deliver the laser radiation to the solid target. Among the laser parameters that can be controlled, we can consider the wavelength, pulse duration, repetition rate, energy per pulse, number of pulses interacting with a single region and the interaction area. As in PLAL, pulsed laser sources with broad frequency spectrum and narrow duration in time are used, is important to keep in mind that during the NPs production the ablation process is not the only one taking place, also some other processes can be promoted between the recently created NPs, the cavitation bubble, the plasma plume and the incoming laser radiation.

Wavelength. One of the first points to consider in the correct choice of the pulsed laser source is the central wavelength. Depending on this parameter is going to be easier or harder for the solid target to absorb energy. Usually, the most of the materials absorb UV radiation by inter-band transitions in an efficient way, thereby increasing the multi-photon absorption and photo-ionization processes leading to narrower NPs size distributions [88]. But in the other hand, the same wavelength range is also strongly absorbed by NPs, therefore recently created NPs can interact with the laser radiation gaining enough energy to end up fragmented or interact with the liquid ambiance species, in this way the NPs size distribution gets wider, the degradation of molecules in the liquid solution can be promoted and the NPs production rate gets diminished due to the investment of energy in the interaction with recently created NPs. Infrared radiation conversely, is mostly absorbed by defects and impurities in the most of the ordered solids and poorly absorbed by nanometer ordered systems, therefore infrared radiation avoid the interaction with recently created NPs leading to narrower particle size distributions and minimal degradation of molecules in the liquid environment, but the fabrication rate gets even lower [88].

Pulse duration. Pulsed laser sources are characterized by concentrating a large amount of photons in short periods of time, comparable with electron-lattice thermalization speed (in the order of ps). If the pulse duration is shorter than ps multi-photon absorption and photo-ionization is stronger, otherwise the conduction of energy to the rest of the solid target is stronger leading to thermal effects [79,91]. Nevertheless it has been observed that when $n s$ pulses are used, a temporal overlap of the plasma plume and the radiation persistence happens, leading to a coexistence in which the laser 
radiation boost the energy of the plasma plume and energetic species located inside it are more likely to evaporate, leading to narrower particle size distributions [92].

Energy delivered by each pulse. Beyond affect the particle productivity rate, the energy delivered in each pulse can also affect the mechanisms in which particles are created. When a higher energy is used, more matter interacts with the radiation and more detachment mechanisms are simultaneously promoted, which leads to have larger portions of energetic species in the plasma plume and making possible to create larger NPs. Therefore, when the energy increases there is a compromise between the maximum particle productivity rate and the widening of particle size distribution [91].

Number of pulses interacting with a single region. When a pulse interacts with a target's surface and ablation is promoted, the surface's morphology is modified due to the extraction of material, and also the composition of the new surface changes due to its interaction with the molecules of the liquid environment. These changes lead to a modification in the absorption of energy making it not homogeneous in the whole radiation interaction area, and affecting in this way the ablation process at each pulse [93]. Therefore, the more pulses hit a localized area, the more ablation mechanisms coexist and wider the particle size distribution gets.

Repetition rate. The repetition rate becomes important when the pulse interacts with phenomenons that happen at large periods of time, this is the case of the cavitation bubble lifetime (in the order of $10^{-4} s$ ) [88]. When the time between pulses is shorter than $k H z$ the pulses interact with cavitation bubble before reaching either the plasma plume or the solid target. The cavitation bubble presents a discontinuity in the refractive index at its interface liquid-gas, leading to scattering of laser source ending up decreasing the energy delivered to the solid target [94]. But up to now, it is still not completely clear the real influence of the overlapping between the radiation and the cavitation bubble since when higher repetition rates are used, higher concentration of NPs near the radiation interaction area are reported [95]. This effect leads to agglomeration of recently created NPs and the further maximization of light scattering and minimization of particle productivity rate [96].

Interaction area. As it was mentioned above, pulsed laser sources concentrate a large number of photons in narrow periods of time, but if the confinement is also extended to the space, the photon's density in a single pulse increases even more making very efficient the delivery of energy. Nevertheless, as it was mentioned before, the excess of delivered energy can promote the widening of particle size distribution. 


\subsubsection{Chemical parameters}

The parameters that are considered chemical, are related to the liquid environment and solid target's structure. Common sense suggests that target's structure can be considered the most important element which is going to determine the final characteristics of the NPs, nonetheless considering the electromagnetic intensities used in PLAL, the liquid environment in which particles are created can have a strong influence on their chemical composition and physical properties, mainly due to the interaction of chemical active species during or after particle creation.

The level of influence of both elements depends on the energy used for NPs creation but also on the chemical reactivity between them at normal conditions. Both elements represent complex systems and should be analyzed in detail separately.

Liquid or colloidal environment. The main important points of the environment's influence that should be addressed lies, firstly on the spacial confinement of plasma plume and cavitation bubble, secondly on the interaction between its chemical species and the NPs formation process, and finally its further influence on NPs agglomeration process.

As it was mentioned above, the biggest attraction of fabricating nanostructures by ablation in a liquid ambiance is the possibility to have a high control over the final material physical-chemical attributes. When a liquid ambiance is used in ablation process instead of a gas ambiance, aside of a cavitation bubble appearance, the density and viscosity is higher and the relief of energy from the plasma plume becomes more difficult, thus precluding an overgrowth of the plasma plume and therefore promoting a more controlled NPs formation [84]. It is also well known that during the ablation process not just target's material is receiving energy, also chemical species in the liquid ambiance does. The energy absorption from chemical species located at the plasma plume promotes in the most of the cases its dissociation, giving the chance to induce impurities in the NPs, but also react with the energetic species detached from the solid target [97]. Normally, regardless purity level of solvents used in the experiments under traditional conditions, the most of them contain atmospheric gasses, being reported $\mathrm{CO}_{2}, \mathrm{O}_{2}, \mathrm{H}_{2} \mathrm{O}$ and $\mathrm{N}_{2}$ the most chemically active for the most of target's materials [98]. The concentration of each element in the liquid solution may promote different chemical effects resulting in important changes on the created nanomaterials. Among the most important results reported up to know, it has been determined that the biggest consequence lies in the stability, size distribution and final morphology of the NPs [90,99]. To have a better understanding of this, it's interesting to analyze the special case of metals, due to the extensive research around them. 
For metallic solid targets, solvents with a high concentration of oxygen and molecules containing it, the most probable result is the oxidation of NPs surface leading to stable colloids. The oxidation of particles may happen at the beginning of their creation, during this time detached energetic species form non-stable systems and have more chances to loose electrons located at the metallic bonds by interacting with ionized oxygen species [100]. This process is not limited to the plasma plume lifetime, also in cavitation bubble can exist the necessary conditions. Contrary to what happens with oxygen species, when a high concentration of carbon is present a different process happens. It has been observed that carbon has a low reactivity with several metals leading to a capping process instead of a reaction. Apparently, as carbonic species don't interact with metallic species, when they coexist in the plasma plume, carbonic species tend to interfere in the NPs growth by forming shields around the energetic species contained in the plasma plume, which limits the NPs growth [101]. Nevertheless, it has been observed that through the time the capping effect of carbonic species also leads to agglomeration of the captured NPs [102]. At this point, it is clear that PLAL is compatible with the use of molecules that act as capping agents, giving the possibility to use dissolution.

The use of dissolution represents endless opportunities, from controlling the particle's size and stability by the use of surfactants to functionalize particles by the use of more complex molecules [90]. In general it has been reported that the use of dissolution can promote interactions with the detached material at different intensity levels.

The first level of interaction is governed by electrostatic interactions, when molecules in the liquid environment don't present a strong chemical reaction with the detached species, tend to form a molecular shield which surrounds the particles limiting the growth and possibly avoiding the further agglomeration [103]. When the molecules in the dissolution present a moderated chemical reactivity with the material's target, chemical bonding with particle's surface is possible allowing a irreversible capping effect promoting the most efficient way to limit the particle's growth among others [89]. When the chemical reactivity between the target's material and dissolution is so strong, the chemical composition and crystalline phase of particles and dissolution's molecules can be modified leading to obtaining exotic chemical species [104].

Solid target. Beyond represent the basic material from which NPs are going to be created, solid target also has a strong influence on the ablation mechanism that is going to promote the detachment of material. Perhaps one of the biggest lessons left by the famous Heinrich Hertz's experiment of photoelectric effect that eventually was 
explained by Albert Einstein is that every element has a minimum photon energy to be ionized. In this sense, the choice of solid target has a direct implication on the energy exchange process between laser radiation and the surface's solid material itself. The values of the photoelectric's energy threshold depends on the crystalline state of the material, on the presence of electrons at the last electronic layers, on the possible mixtures of materials, and finally the Fermi's energy in the system [105]. Normally, transition metals like $\mathrm{Au}$ or Ag present the highest energy threshold and alkaline metal present the lowest energy threshold [106].

At lower energy thresholds, electron detachment due to the absorption of a single photon increases prioritizing the photo-ionization effect over electron-lattice thermalization, preventing in this way the investment of energy in thermal effects during the ablation process [107]. Additionally, the increment of species coming from photoionization in the plasma plume can promote a higher density of energetic electrons in the plume zone leading to non-equilibrium conditions in the plasma plume. A strong energetic imbalance in the plasma plume can result in the formation of new materials or materials with non-stable crystalline phases [108].

\subsection{Post-irradiation process to control nanomete- rials characteristics after PLAL}

PLAL synthesis route, offers the possibility to obtain high quality materials with characteristics hardly achievable by other synthesis routes. However, a further control over nanomaterials is always possible, either by chemical or physical processes. Among the traditionally used methods, is possible to use external chemical agents to promote reactions that lead to changes in the nanomaterial's surface, the selection of specific nanomaterials sizes or morphologies through centrifuging or similar physical methods [103]. Also the application of external electromagnetic fields together with the introduction of chemically active elements is used to promote functionalization of the material. Nevertheless, the most of the conventional methods lead to further negative secondary effects like agglomeration, generation of chemical waste, among others [109]. To face this issues, PLAL enthusiast groups decided to approach the topic by a familiar perspective. Replacing the solid target in PLAL technique with the recently created suspension as it is represented schematically by Figure 2.5, some very interesting phenomena that lead to further modification of the material can be observed. The nanostructures tend to undergo to melting, evaporation or fragmenta- 
tion leading to changes in phase, chemical composition, and morphological and size distribution [110].

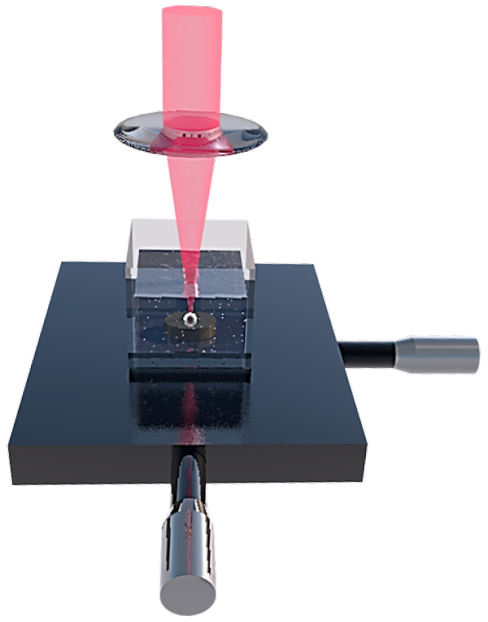

First Stage

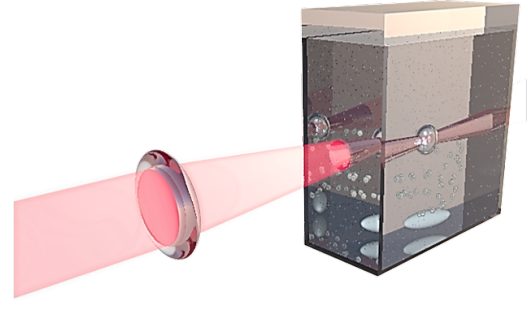

Second Stage

Figure 2.5: Synthesis approach considering a first stage where NPs are created by PLAL and a post-irradiation stage where the nanomaterials are post-processed.

When intense pulsed laser radiation interact with nanostructures suspended in liquids, three main processes are promoted: Thermal processes that leads to the melting or evaporation of the material, electronic detachment of the particles leading to Coulomb explosion and fragmentation, and finally near-field ablation.

Melting mechanism. When a colloid is irradiate by pulsed laser radiation, the principal interaction mechanism is the absorption of energy by both the liquid environment and the solid material suspended in the liquid. When the energy exceeds a certain threshold and is being continuously absorbed by the system at similar times for the needed to the system to release energy, no matter if the incoming photons have enough energy to pull out single electrons from atoms located at the nanostructures surface, the entire structure will receive enough energy to induce a fast vibrational move of electrons in the atomic lattice causing the melt of the structure as it is depicted in Figure $2.6[111,112]$. After this process, when the liquid droplet releases the excess of energy to the rest of the environment medium, tend to solidify in a spherical shape. When the energy exceed by far the threshold, not just melting can be promoted, also evaporation of the system's surface may happen [113].

The energy threshold at which this process happens is governed mainly by two material's characteristics: its size and chemical composition. Structures with big- 


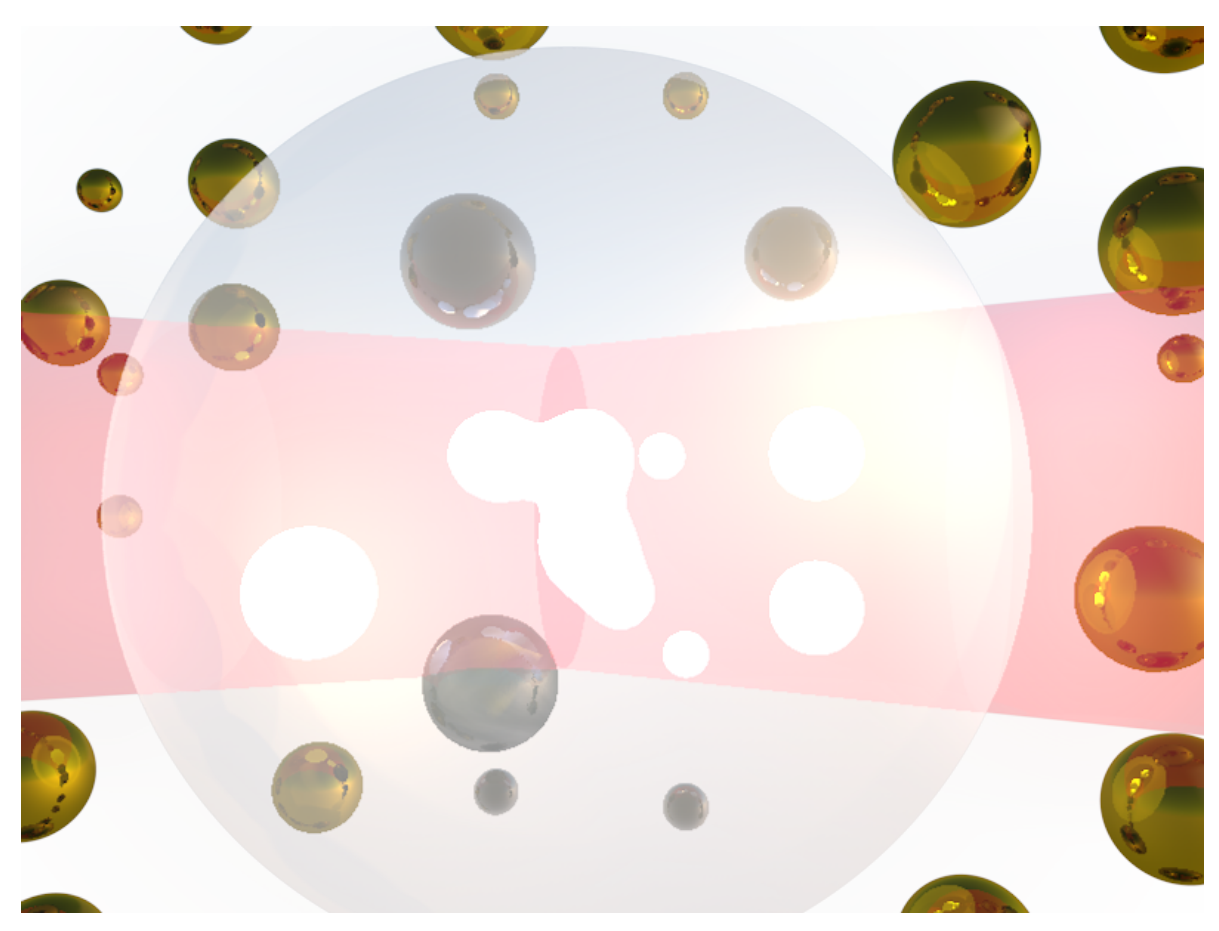

Figure 2.6: Melting mechanism in post-irradiation method where droplets of nanomaterial are formed due to the fast vibrational move of the electrons in the NPs, which is a consequence of the absorption of laser radiation in beam's focus area.

ger sizes demand a bigger investment of energy to induce the thermalization of the electronic lattice [114]. But also structures with stronger ionic and molecular bonds demand bigger amounts of energy to achieve the same state [115]. Another important parameter to consider is the absorption mechanism promoted by the material. In some special cases optical nonlinear or linear effects can be present influencing the way in which the material is going to absorb energy. To be more precise, one of the most important of this effects is Surface Plasmon Resonance (SPR) present in the most of the metal NPs, promoting a selective absorption of energy as a function of NPs size [116]. This phenomenon is going to be discussed in detail in the 4th chapter.

An important result should be highlighted. When a colloid containing more than one solid material is irradiated and the melting mechanism is promoted, is also possible to obtain homogeneous alloy by reaching the threshold energy levels of both elements, as it was demonstrated by Izgaliev and co-workers [117], whom reported the fabrication of silver/gold alloy in water. Considering that silver and gold nanoparticles with the same size present a similar lattice constant and absorb energy radiation at similar frequencies, their energy threshold was similar making possible to get the homogeneous alloy. 
Coulomb explosion mechanism. In contrast with melting mechanism, Coulomb explosion is caused by multi-photonic absorption and kinetic energy transfer [118]. In this case, similarly to what happens with PLAL, huge amounts of electrons are pulled out of the nanostructures surface due to photoelectric effect, thermionic emission or photo-ionization leaving a lack of electrons in the system, which leads to an excess of positive charges in the system's surface which results in an increment of electromagnetic repulsion force in the whole system [119]. The most probable way to get rid of the excess of energy is by dissociation of the whole system into smaller but stable systems. This process is considered a form of Coulomb explosion.

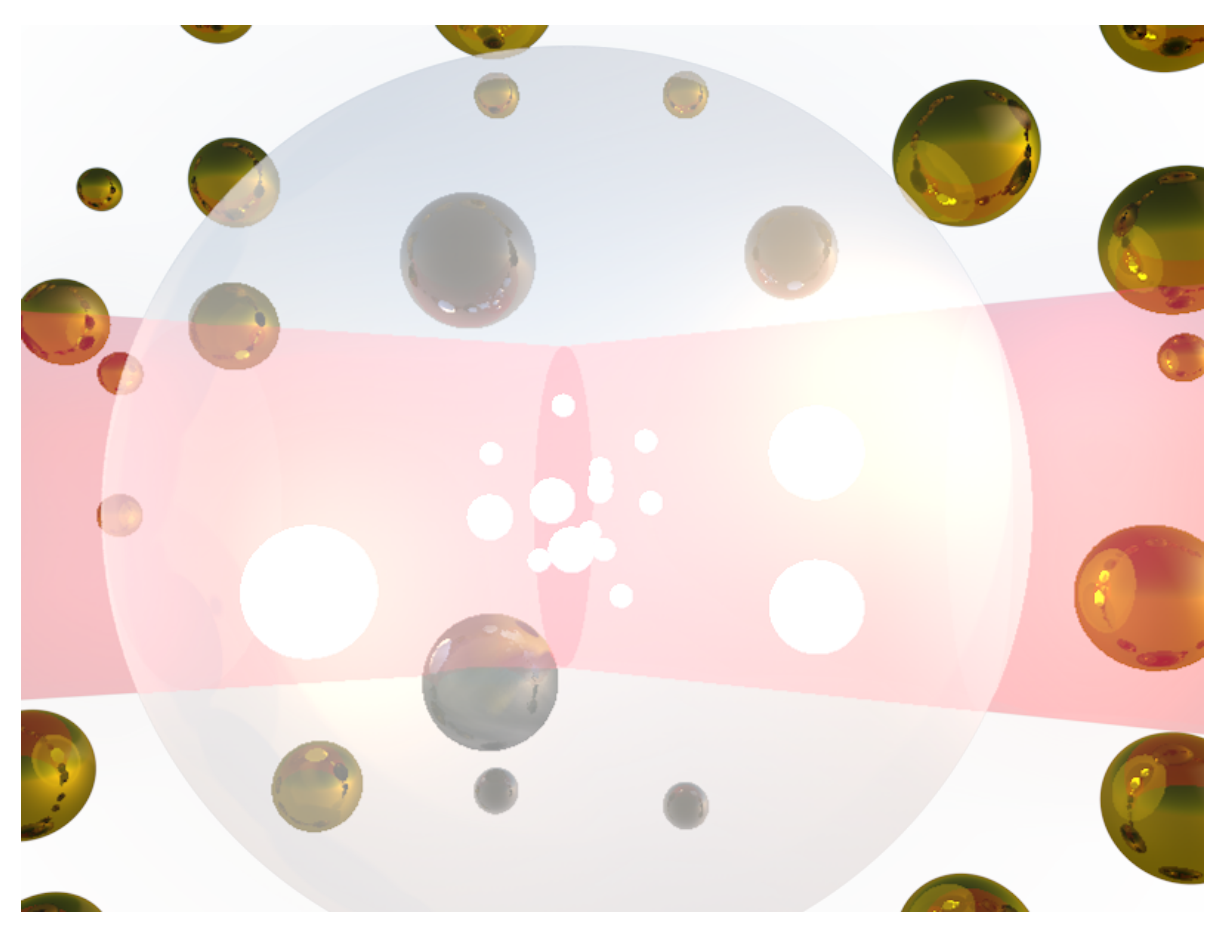

Figure 2.7: Coulomb explosion mechanism in post-irradiation method where small pieces of nanomaterial are formed due to a dissociation of original NPs, which is a consequence of the absorption of laser radiation in beam's focus area.

Up to now is not completely clear, but is believed that the principal condition for Coulomb explosion to happen over a melting mechanism is the speed of the absorption process and the amount of energy used. Temporal long pulses preferably promotes electronic-lattice thermalization, but pulses in the temporal region under ns are more likely to promote electronic effects without giving the chance to spread the energy to the electronic lattice [120]. In the other hand, when the energy delivered to the colloids is far above the melting energy threshold, not just thermal effects can be promoted. 
Near-field ablation. Beyond melting mechanism and Coulomb explosion, it has been proposed an alternative mechanism when the energy delivered to the colloid is extremely high, a near-field mechanism. Similar to the PLAL case, when the colloid is irradiated at high intensities, the solid systems tend to absorb the incoming energy by either multi-photonic absorption, kinetic energy transfer by collision, or by electron-lattice thermalization. In any case, when the amount of absorbed energy is so high and happens in a short period of time, i. e. in a time under the needed time to release energy, the solid systems tend to get unstable and get dissociated into energetic species like electron with a high speed, ions, another unstable but smaller solid systems and smaller but stable solid systems. In general the whole entity interacts with other energetic species coming from the liquid environment and other nanomaterials promoting the creation of a plasma, the plasma interacts with other solid systems in its vicinity leading to the dissociation of more solid material [121]. However, some groups have demonstrated that metal NPs are able to melt at high intensities without suffer fragmentation [115]. But metal nanostructures like gold NPs should present a high field enhancement promoting the emission of ions leading to Coulomb explosion, and allowing the possible formation of a near field plasma at high intensities. Thus, the near field ablation mechanism require further studies to be considered as a real alternative for the most of the materials.

At this point, should be clear for the reader that all of this interactions are not isolated processes and in all of them are present the following process: dissociation of liquid environmental molecules and its subsequent mixture with energetic species resulting in appearance of crystalline defects in recently created nanometric systems [122], inducement of cavitation bubbles due to the release of energy from the energetic species to the rest of the colloidal environment [123], and the induction of shock waves traveling through the rest of the colloid promoted by the extinction of the cavitation bubbles as a release of energy [124].

Final remarks. Finally is important to notice that, to trigger any of these effects, the amount of energy investment should be equal or exceed a threshold, the threshold is depending on the chemical composition, crystalline phase and size of the nanometric systems. The databases that reflect these values remain poor and further research is needed in the field to enlarge these databases. In the other hand, although the influence on the length of the pulses is not fully understood, some theoretical approaches try to clarify it. In this context, Werner and Hashimoto [125] have developed a thermodynamic approach to understand the processes happening through the time when gold NPs suspended in a water environment are irradiated 


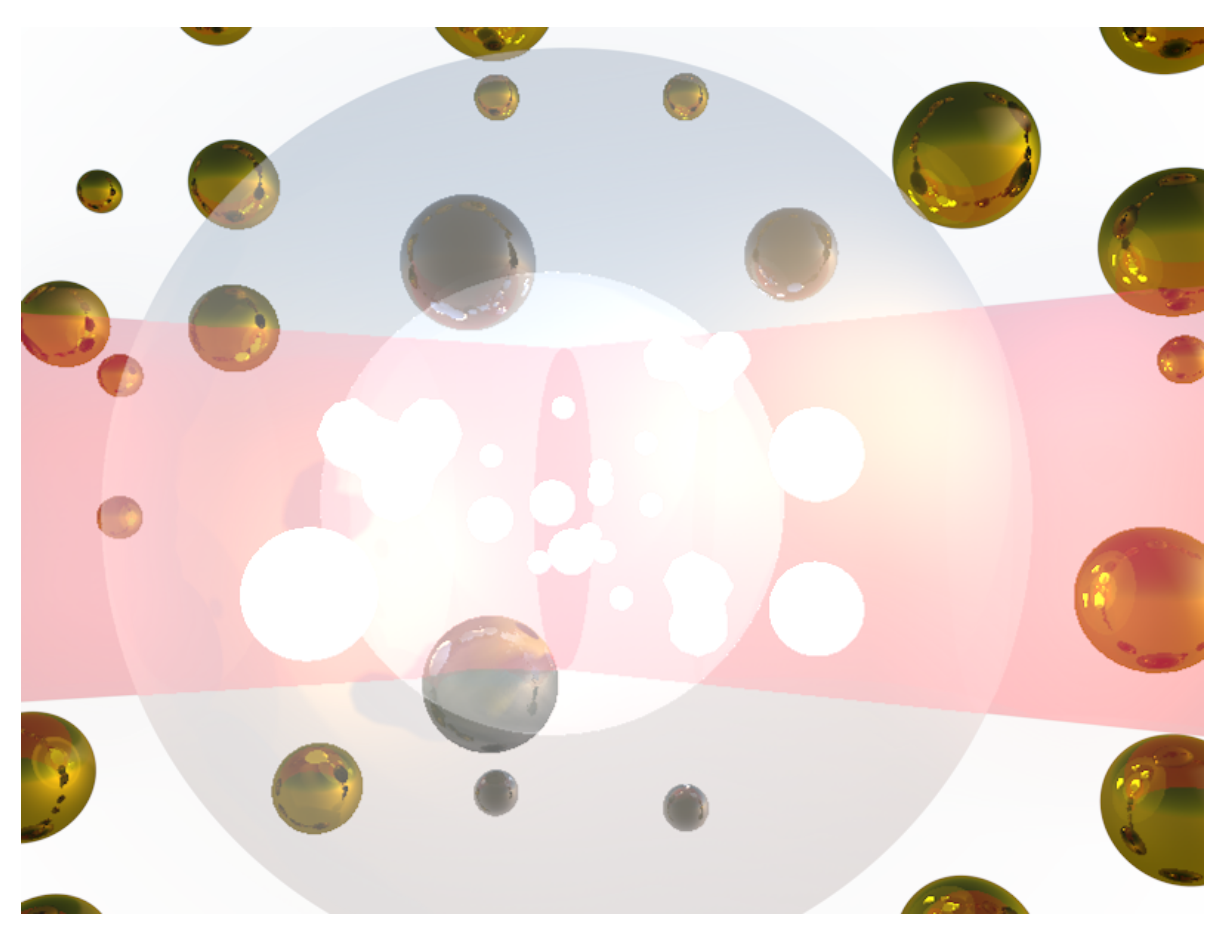

Figure 2.8: Illustration of near-field plasma mechanism in post-irradiation method, where a plasma plume appear in the beam's focus area due to the absorption of hogh intensity laser radiation.

by fs, ps and ns pulses. The approach considers the size of NPs, laser energy used to irradiate the particles, and laser wavelength. After analyzing the evolution and interaction of the specific capacities of electrons, electronic-lattice, and medium, the model helps determine the dynamics of the transmitted temperature from electrons to the lattice and then to the medium. This analysis may help to understand the appearance of different mechanisms in which matter react at different energy thresholds. Nevertheless, even this theoretical treatments are still not completely accurate, mainly due to a lack of understanding about what happens after appearance of effects derived of the release of energy, as the cavitation bubbles or the expansion of shock waves. However, is important to point out that a complete theoretical approach which describes the phenomenons promoted by a post irradiation of colloid created by PLAL is still needed to fully understand the entire scope of this technique. 


\section{Chapter 3}

\section{Characterization techniques}

A unique aspect of matter when is reduced to nanoscale, is an increase in the surface to volume ratio and in many materials this promotes the emergence of new mechanical, thermal, optical, magnetic effects, among others [126]. The change in the effects is mainly due to, the electrons that form these systems experience a quantum confinement and their behavior is altered.

The analysis of the electronic behavior of each element of a whole atomic system can not be achieved in an accurate and general way, mainly because this reflects both its self behavior and the influence of each element that form part of the system, therefore the entire system should rather be analyzed. To make a fair interpretation of the properties present in nanomaterials, we need to understand what characteristics are sought. In other words, when new materials are produced, it is important to know what kind of features are interesting to get. For instance, in metal nanoparticle creation several properties are present, in particular the characteristics that have been more explored are optical and electromagnetic [55]. However, there are behaviors present in these particles that have been poorly studied as their thermal or catalytic response [127], but may represent significant progress in areas where the use of NPs is still not too extended, as catalysis, energy harvesting, among others.

For this thesis work, the idea to create NPs by PLAL was to try to exploit its unique manufacturing characteristics, such as high purity of the material, the possibility of obtaining nanostructures in-situ in complex liquid environments, the ability to obtain ligand-free nanometric materials and finally the possibility to create nanostructures with important optical and thermal characteristics.

To analyze the created materials during this thesis work, and understand their influence on the environment in which they are created and their potential for use in real applications, several techniques that don't promote further modifications are 
used. In particular these techniques can be divided into three groups, optical, X-ray and electron-based and thermal techniques.

\subsection{X-ray and electron-based techniques}

Techniques based on the use of high intensity electromagnetic radiation propagating with a wavelength comparable to atomic dimensions, or being more precise, on the irradiation of samples with X-ray beams, can be used to obtain accurate information on the arrangement of atoms in crystal lattices, due to the X-ray wavelength dimension, create diffraction patterns when X-rays interact with a crystalline sample. Also it can be used to create images of samples with a resolution of nanometers for a visual interpretation of structures.

The formation of diffraction patterns is a consequence of an elastic dispersion of $\mathrm{X}$-rays after interacting with the electrons of atoms organized in a periodic arrangement, and its further interference [128]. The constructive and destructive interference promotes the creation of the patterns, which characteristics are on function of the separation between atoms and the dimensions of atoms. Traditionally, when a X-ray beam hits an atomic lattice at an angle $\theta$, Bragg's law is used to predict if the X-ray wave experiments a constructive or destructive interference and is expressed by the following equation [129]:

$$
n \lambda=2 d \sin \theta
$$

Where $d$ represents the distance between atoms forming different sets of arrangements known as crystalline planes, $n$ represents an integer which corresponds to the matching of one or more waves, and $\lambda$ represents the wavelength of X-rays. Bragg's Law makes clear the periodic arrangement of atoms in a solid and helps to understand that there are different atomic arrangements. Nowadays, it is still used to determine if a certain sample present or not a periodic arrangement. In many cases this characteristic helps differentiate between materials, or phases of the same material. A change in the periodic arrangement results in different chemical and physical response due to the forces present between atoms [128].

To obtain diffraction patterns that lead to the analysis of the periodic structure or lack of it in materials, the simplest device used is called diffractometer. Particularly in this thesis work, it was used a Siemens D5000D powder X-ray diffractometer with two pressure gauges, tube $\mathrm{Cu}$, diffracted beam monochromator, and scintillation detector. 
The use of this instrument was fundamental to determine if after PLAL the samples present the same crystalline phase or it have changed.

For a better understanding on the crystalline analysis performed, is mandatory to review some important concepts like crystal, crystalline plane, and Miller index. A crystal is an stable system which is formed of a periodic arrangement of molecules or atoms in 3 dimensions. Each node is identical and the most basic parallelepiped is known as unit cell, the cell can be described by the three different lengths in the spatial axis, and in the three different angles formed between the axes. Each face of the parallelepiped or a simple plane which cross an axis of it and contains at least one node is the base of a two dimensional arrangement in the crystal known as crystalline plane. In a three dimensional periodic structure, these planes are parallel separated by a constant length known as inter-planar distance [26]. A group of parallel crystalline planes is mathematically identified by three numbers $h, k$, and $l$. These numbers help to identify the number of intersections of crystalline planes with the axis of the unit cell and are known as Miller's index in honor of its discoverer. These index also help us to determine the geometry of the crystal structure in our system. The relations for the most conventional crystalline geometries are the following:

$$
\begin{gathered}
\text { Cubic } \frac{1}{d^{2}}=\left(h^{2}+k^{2}+l^{2}\right) \frac{1}{a^{2}} \\
\text { Tetragonal } \frac{1}{d^{2}}=\left[h^{2}+k^{2}+l^{2}\left(\frac{a}{c}\right)^{2}\right] \frac{1}{a^{2}} \\
\text { Hexagonal } \frac{1}{d^{2}}=\left[\frac{4}{3}\left(h^{2}+k^{2}+h k\right)+l^{2}\left(\frac{a}{c}\right)^{2}\right] \frac{1}{a^{2}} \\
\text { Orthorhombic } \quad \frac{1}{d^{2}}=\frac{h^{2}}{a^{2}}+\frac{k^{2}}{b^{2}}+\frac{l^{2}}{c^{2}}
\end{gathered}
$$

Where $d$ represents the inter-planar distance, and $a, b, c$ represents the three spatial distances in the axis of the unit cell, or being more precise, the first three inter-atomic distances presented in the geometrical shape of the crystal.

The information that can be obtained from these parameters is the inter-atomic separation, lack of atoms in each node of the crystal, if the separation between planes is a constant, if there are crystallographic planes that do not correspond with the material under study, etc. In other words, X-ray diffraction helps to determine if the sample suffers any crystalline change after PLAL, if the NPs don't present crystalline defects, or how similar are the nanomaterials to the bulk material [130]. 
The structural studies are not limited to analysis of results coming from diffractometers. In particular, in this thesis, more sophisticated instruments and techniques were also used to achieve more accurate results and better interpretations. To this end, a transmission electron microscope JEOL 2100 thermionic gun lanthanum hexaboride $\left(\mathrm{LaB}_{6}\right)$ which works at a voltage of $200 \mathrm{KV}$ maximum acceleration, with a system of energy dispersive micro-analysis Enegy INCA TEM 200 Oxford, was used for micro-structural examination of specimens, crystallographic study by electronic diffraction, determination of crystal planes, and compositional analysis of the samples. And also the determination of particle size and distribution, could be measured using the same instrument. The techniques used were Transmission Electronic Microscopy (TEM), Energy Dispersive X-ray Spectroscopy (EDX), High Resolution Transmission Electronic Microscopy (HRTEM) and Selected Area Electronic Diffraction (SAED).

TEM, just as in light microscopy, a beam is directed to a sample through a set of lenses to form an amplified image of the sample. But in this special case, the beam is formed by accelerated electrons, the lenses are electromagnetic, the sample is seen under vacuum conditions, and the collision of electrons with the sample promotes the formation of an image which is an interpretation of the measurement of scattered electrons in different directions in a solid angle. The resolution that can achieved with this type of microscopy is on the atomic scale, mainly due to the spatial dimensions of electrons and how they propagate [131]. Generally the accelerated electrons propagate in waves, with a length commonly known as de Brogli wavelength, this length is in the atomic range and as a result, electrons passing through the inter-atomic space allow the formation of high-resolution images where it is perceptible the position of each atom in a crystal, as it can be appreciated in Figure 3.1. By the use of this technique, it is possible to determine the morphology, size, size distribution and spatial distribution of created nanomaterials. And at the finest resolutions is also possible to determine if the material presents a periodic ordering, chains of atoms which represent a crystalline plane, distance between crystalline planes and the presence of crystalline defects [132].

Additionally, considering that by irradiating the sample with accelerated electrons, some of them instead of being transmitted at a certain angle are reflected, or rather back-scattered. This phenomenon can be exploited to perform diffraction analysis since these electrons propagate at wavelengths similar to the X-ray. In this sense, SAED presents the possibility to study the crystallographic properties of a sample but not as a whole, micro-analysis of selected areas can be performed.

The diffraction patterns studied in SAED are not so similar to those obtained in Xray diffraction. Using a diffractometer, an X-ray beam irradiate a powder sample and 


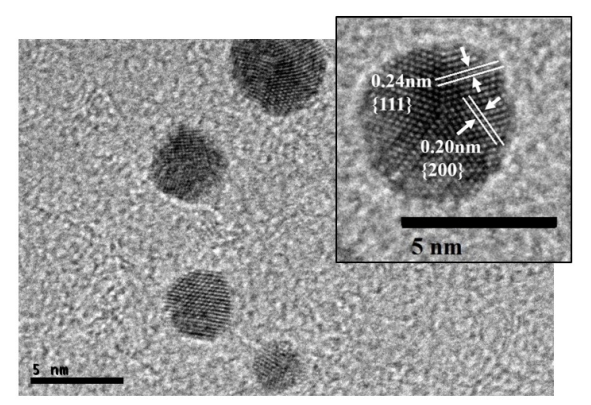

Figure 3.1: High Resolution Transmission Electronic Microscopy Picture taken of one of our gold NPs samples.

the dispersed X-ray waves interfere with each other constructively of destructively, a detector captures the signal variations and the diffraction patterns translated as a graphic of intensity vs angle at which the beam irradiates the sample. In SAED, the beam is not moving a $\theta$ angle, instead of that, the electronic detector creates an image in two dimensions of the interference created by the back-scattered electrons. These electrons interact with the sample in a circular area and their interference is a result of electronic waves dispersed in the three spatial axis. Generally, the detectors used to interpret an image created by electrons are charge-coupled devices (CCD). These devices represent either the normal image of the sample or the image in the Fourier space, making possible to visualize chains of atoms as points, similar to Fourier's transformation where constant lines in the time domain can be transformed into diffraction orders in the Fourier space. Therefore, a set of parallel planes is seen as dots, and a combination of different crystalline planes that are perpendicular to each other is represented as a dot grating as it is depicted in Figure 3.2, and each diffraction order corresponds to a single crystalline plane family which can be represented by Miller angles.

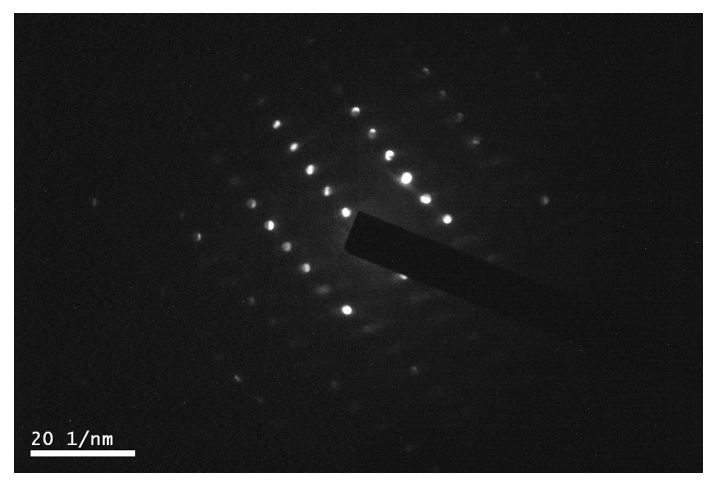

Figure 3.2: Selected Area Diffraction Pattern picture taken of one of our gold NPs samples. 
The correct interpretation of this pattern lies on the measurement of distances between diffraction orders. As each point represents a crystalline plane family, the distance between first and secondary neighbours should correspond to the crystalline plane families of faces of the crystalline unite cell and planes crossing the unit cell's axis containing more than one node respectively. Is also important to notice that the direction of the diffraction grating and distances between points provide information of the observation crystal plane. In some cases this study can provide better resolution when changes in phase are sought [133].

Moreover, the ability to focus the electron beam in a certain area not only helps us to make crystalline analysis, but allows also to perform elemental analysis using an energy-dispersive spectrometer. When an electronic beam hits the sample not all of the electrons are dispersed or transmitted trough the sample, some of them also collide with electrons in the atomic orbit, during the collision the accelerated electrons transmit kinetic energy to the electrons in the atomic orbit, this excess of energy helps the electrons to jump to the next discrete atomic energy level. Nonetheless, as it has been mentioned above, when an electron that is part of an atomic system is excited and jumps to next energetic level, it tends to go to the ground state after some time, and the easiest way to go back to the ground state is liberating the excess of energy through the emission of a photon. When the electrons that are excited belong to the most external orbit in the atoms, the difference of energy between the ground and excited state is the range of X-ray photons. The electronic collisions that promote the excitation of the atoms in TEM, commonly lead to emission of X-rays. However, every element have a different atomic structure and the release of energy through X-rays varies from element to element giving the opportunity to identify each element contained in a sample under study. The name of this kind of characterization technique is EDX [134].

The last X-ray-based technique used in this thesis is X-ray Photoelectron Spectroscopy (XPS). The main goal of the technique is to determine the composition and chemical state of the elements present on the surface of solid materials. The way to achieve this kind of analysis is irradiating the surface of a solid sample with electromagnetic radiation coming from an X-ray source, the exposure to the radiation promotes photo-ionization, and the electrons coming out of the material's surface with a certain kinetic energy $E_{k}$ are collected and measured. The kinetic energy $E_{k}$ is related to the incoming energy by the Einstein's relation $E_{k}=h \nu-E_{b}$ where $E_{b}$ represents the bonding energy of the atoms present in the surface. In this way, by measuring the kinetic energy of the detached electrons is possible to determine 
the electronic structure of the atoms in the sample and infer the chemical state of the samples. The instrument used for this purpose was a Kratos AXIS ultra DLD spectrometer.

To prepare the samples for the electron microscopy technique measurements and XPS, a droplet of the colloidal suspension created in each experiment is dispersed onto a carbon-coated copper-based TEM grid or silver-based XPS grid. The liquid content is then removed with the help of an absorbent paper so the solid particles can remain in the grid surface. This technique allows obtaining dry and dispersed particles, necessary experimental conditions for an easy measurement acquisition.

\subsection{Optical techniques}

As it was mentioned above, in this research work the fabrication of materials with important optical characteristics was fundamental. Therefore, the optical characterization of the samples was mandatory and the most of the optical characterization techniques are centered in analyze the optical response of the specimens. However, when nanomaterials in liquid environments are under study optical characterization techniques are not limited to provide information of interesting optical features of the samples, also can provide information of the size, size distribution, and stability of colloidal samples.

The techniques used to extract information from the samples created by PLAL are based on three linear optical phenomena, absorption, transmission and scattering of light. The three of them are deeply interconnected and show precise information that helps to distinguish one sample from another.

It is intuitive to know that when an element hindering the transmission of light, this interact with the element. All physical elements are made of atoms containing electrons orbiting around. When light interacts with any physical element, such interaction occurs from the influence of the electromagnetic field of the light on the movement of electrons that are part of the physical element. The first and most basic interaction happening is that the electrons orbiting atoms of the element that hinders the passage of light follow the direction of electric field of light, leading to an oscillatory movement [10]. If radiation contains photons with energies comparable to energetic differences between atomic orbitals which are characteristic of the material, these photons will be absorbed by the electrons promoting an energetic jump, and when they return to their ground state the most likely way to do this will be liberating the excess of energy by emitting a photon but in a different direction of the 
absorbed photon. Photons that in its path don't interact with any energy absorber will continue their trajectory without experiencing changes. Photons that do not match the required energies to be absorbed by electrons in the material and promote an energetic stable jump, also can be absorbed but promoting energetic jumps to meta-stable or non-stable energy levels and the most probable way in which electrons are going to get rid of the excess of energy is through vibrations and not emitting photons, making in this way possible for other photons to be absorbed and reach higher energetic levels and giving the chance to promote the emission of photons with higher energies. Among others, this processes summarize the most probable way in which the matter is going to interact with the incoming radiation.

Taking this as starting point, it is clear that due to the separation between atoms in the various phases in which matter can be found, radically different interactions can be distinguished mainly by interactions that can be suffered by a solid, liquid or a gas. For gas, transmission of light is dominant, the absorption for solid and more or less balanced absorption, transmission and dispersion for liquids. Nevertheless, when speaking about colloid composed by liquids and nanoscopic solids these interactions may be more complex [135].

In this context one of the most interesting processes in nanostructured colloids is the SPR. This phenomenon consist in a discontinuous absorption of the visible electromagnetic spectrum. The process is mainly important and widely explored in bio-medicine, due to the possibility to absorb light in the spectrum region of wavelengths that are not commonly scattered by living tissue [136]. The process is as follows: Generally, metal nanomaterials tend to have many of their electrons on the surface and due to the kind of bonds present in these materials (metal bonds), electrons experience a relative free mobility along the entire surface of the metal. When this structure interact with a source of light, not necessarily a coherent light source, the electrons located at the surface are going to absorb the incoming radiation or follow the field of the incoming electromagnetic waves, as the electrons have a relatively free mobility, when the incoming radiation has the necessary energy, the electrons located at the surface are going to follow the direction of the field oscillating all together. The movement is known as plasmon and it can be interpreted as a non radiative wave which propagates in a direction parallel of the materials interface, as it is represented in Figure 3.3. Since the movement happens at the interface between the solid and another medium like liquid or gas, the oscillations are extremely sensitive to the dielectric function of the solid and the medium in which the solid is contained. Furthermore, in nanomaterials the oscillation is also extremely related to the system's 
size and shape, since at this scales the spatial confinement induces an electronic potential in the systems which result in a discretization of energy levels of the electrons and a restriction on their possible moves. Therefore at different sizes and shapes, the nanostructure allows the electronic oscillation for different wavelengths [137].

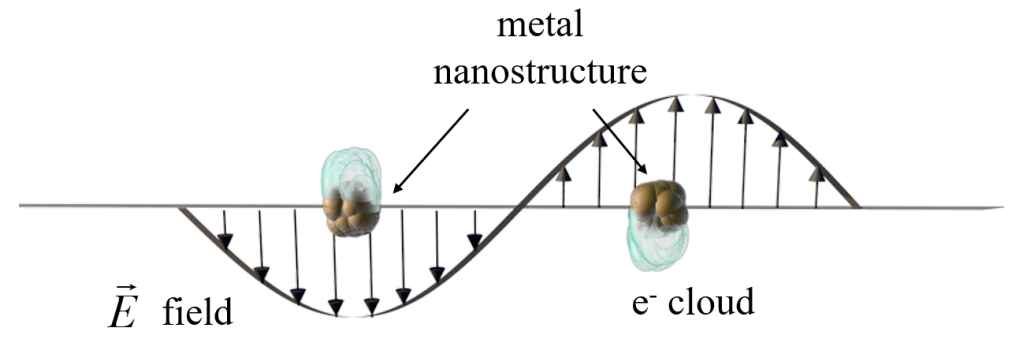

Figure 3.3: Schematic diagram of SPR in a metal nanostructure.

From a practical point of view, this phenomenon can be used to determine the size and morphology of nanomaterials in a macroscopic way or determine the amount of solid material presented in a colloid, etc [138]. For this thesis work, SPR was exploited to allow the absorption of energy in regions of the visible spectrum and to determine the stability of colloids created by PLAL.

To measure the absorption of the created materials, we have used a Spectrophotometer Cary 300 UV-Vis, equipped with dual beam, Czerny-Turner monochromator, $190-1100 \mathrm{~nm}$ wavelength range, approximately $1.5 \mathrm{~nm}$ fixed spectral bandwidth, full spectrum $X e$ pulse lamp single source, dual $S i$ diode detectors, scan rates up to $24000 \mathrm{~nm} / \mathrm{min}$, 80 data points per second maximum measurement rate, nonmeasurement phase stepping wavelength drive, and room light immunity.

Commonly the measurements are taken as follows: A particular wavelength is selected using the monochromator and this light is directed to a chamber where the sample is contained in a quartz cuvette that allows $90 \%$ of light transmittance at a large range of wavelengths from $200 \mathrm{~nm}$ to $2500 \mathrm{~nm}$. The light propagates through the sample and the radiation transmitted in the direction of the original beam is collected by a detector. The intensity of the incident radiation and the intensity of the transmitted radiation is compared and the rate of intensities is reported. By the same procedure for a wide range of wavelengths, is possible to obtain a transmission spectrum. From the transmission spectra is possible to determine the transmittance, the absorbance, and the absorption coefficient of the samples through the following relations: 


$$
\begin{gathered}
T=\frac{I}{I_{0}} \\
A=-\log _{10}\left(\frac{I}{I_{0}}\right)=-\log _{10}\left(\frac{I_{0} e^{-\alpha(\lambda) d}}{I_{0}}\right)=\frac{\ln \left(e^{\alpha(\lambda) d}\right)}{\ln (10)}=\frac{\alpha(\lambda) d}{2.302585}
\end{gathered}
$$

Where $I_{0}$ represents the intensity of the incident radiation, $d$ represents the optical path that light should interact with the sample, $\lambda$ represents the wavelength with which the sample is irradiated, and $\alpha$ represents the absorption coefficient. Transmittance $T$, and absorbance $A$ are related through the following equation.

$$
A=-\log _{10}(T)
$$

This relationship helps to establish mathematical parameters that can then be used to graphically represent the optical response of the materials under study, as it is shown in Figure 3.4.

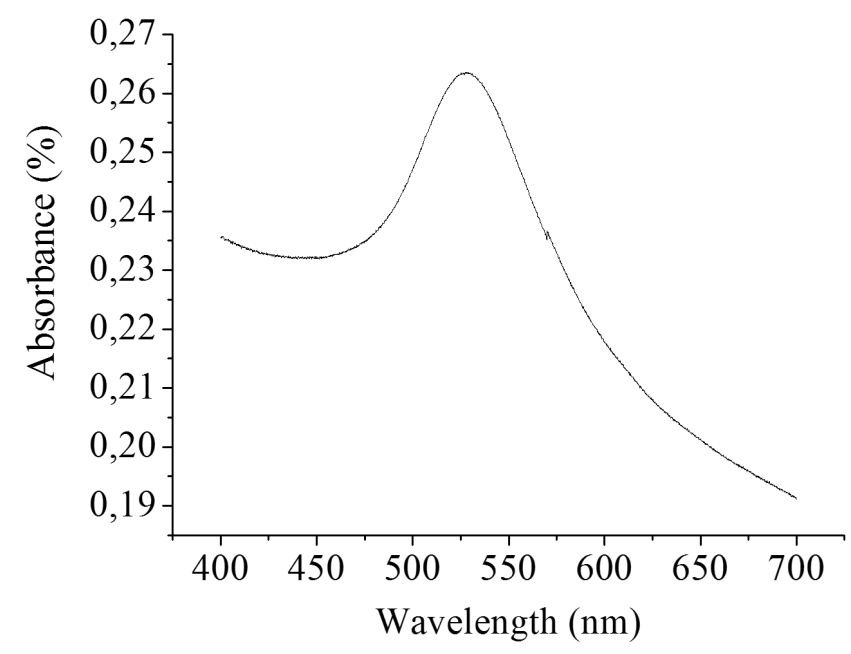

Figure 3.4: Characteristic spectrum of gold NPs obtained during the experiments performed in this research work.

The analysis of an absorption or transmission spectrum of a colloid comprised of nanoscale structures, provides information on the percentage of solid materials interacting with light path through the determination of the absorption coefficient, and the transmission obtained at each wavelength. However, the information related to the size and morphology of the nanostructures can be extracted using a more complex analysis. For this, it is often used the Mie theory based on the solution 
of Maxwell's equations for electromagnetism, when a metal sphere immersed in an ambiance is under an electrical external field. The theory predicts the exact fraction of absorbed light and the fraction of light which was scattered. The sum of these values is the optical extinction due to the presence of the nanostructure [139]. For spherical metal particles with a dielectric function $\varepsilon_{m}$ immersed in an homogeneous medium with a dielectric function $\varepsilon_{d}$, the extinction coefficient is represented as follows:

$$
C_{\text {ext }}=\frac{24 \pi^{2} R^{3} \varepsilon_{d}^{3 / 2}}{\lambda} \frac{\varepsilon_{m}^{\prime \prime}}{\left(\varepsilon_{m}^{\prime}+2 \varepsilon_{d}\right)^{2}+\left(\varepsilon_{m}^{\prime \prime}\right)^{2}}
$$

Where $R$ represents the particle's radius, $\lambda$ the wavelength of incoming radiation, and $\varepsilon_{m}^{\prime}$ and $\varepsilon_{m}^{\prime \prime}$ are represented as follow:

$$
\begin{gathered}
\varepsilon_{m}^{\prime}=\varepsilon^{\infty}-\frac{\omega_{p}^{2}}{\omega^{2}+\gamma^{2}} \\
\varepsilon_{m}^{\prime \prime}=\frac{\omega_{p}^{2} \gamma}{\omega\left(\omega^{2}+\gamma^{2}\right)} \\
\gamma=\frac{v_{F}}{L_{b u l k}}
\end{gathered}
$$

Here, $\omega_{p}$ represents the plasmon resonance frequency, $\omega$ the frequency of incoming radiation, $\varepsilon^{\infty}$ the dielectric function at high frequencies that in the most of the materials can be considered as $1, v_{F}$ Fermi's velocity, and $L_{b u l k}$ the free path of electrons in the macroscopic material. Through these relationships, is possible to fit a theoretical curve to the characteristic spectra of colloids composed by metal nanoparticles and thus determine the radius of the particles. In the case when the particles are not spherical, the absorption peak can be either not present or more than one peak can appear, depending on how many spherical shapes the nanostructure present. In this case, the $R$ can represent the radius of each spherical shape presented in the nanomaterial [140].

UV-Vis Spectrometry is a powerful tool for determining optical and mechanical characteristics of nanostructures, such as SPR position, stability through the time, estimation of the size and morphology, and the determination of absorption range. Nevertheless, phenomenons like scattering can be also exploited to get more accurate information of the particle's size and its dynamics through the time.

The technique used to determine this information is commonly known as Dynamic Light Scattering (DLS). As its name revels, it is based on the measurement of the intensity of scattered light when a coherent light beam encounters in its path an object 
with nanometer dimensions. To be more precise, in DLS brownian motion of particles is measured by analyzing the intensity fluctuations in the scattered light [141].

When a colloid is irradiated with a laser beam, all the particles suspended in the liquid scatter the incoming radiation in all of the directions and the scattered waves can interfere with each other. If a detector is placed at a certain angle in which is able to catch the dispersed light, the interference of those waves will result in an interference pattern with dark and bright zones. Since the particles that promote the scattering are not equally separated, the interference pattern will not show a periodic behavior but rather a random one, these patterns are known as speckle patterns. In colloids where the particles are not stationary, the speckle pattern changes through the time making the detected intensity fluctuate. The rate of intensity change is directly related to the change rate of the speckle patterns, and this is directly related to the particle velocity. The movement of solid particles suspended in liquids, is due to random collisions with molecules form the liquid, this movement is known as Brownian motion. An interesting characteristic of this movement is that smaller particles move faster, making possible to determine the size of the particles through the Stokes-Einstein equation [142].

$$
R=\frac{\kappa T}{6 \pi \eta D}
$$

Where $R$ represents the radius of the particle, $\kappa$ Boltzmann's constant, $T$ the absolute temperature, $\eta$ the dynamic viscosity of the liquid environment, and $D$ represents the diffusion coefficient. In DLS, the diffusion coefficient can be calculated by fitting and exponential curve to a correlation curve measured by the detector. The correlation curve express how similar are the intensities of the collected speckle patterns through the time. For instance, if a particular intensity signal from a speckle pattern is taken at a time $t$, the following signal taken at a very short time difference is going to be practically the same and therefore, its correlation with the first one is going to be so high. In the other hand, a signal taken at a very long time difference with the first one is going to be so different than the first one and therefore, its correlation is going to be so small. Speckle patterns change so fast when they are produced by NPs moving in liquids (at a microsecond scale), and the representation of a perfect correlation reaches the value of 1 , when we continue measuring the correlation at different times in a microsecond scale, the correlation eventually will reach the value 0 and therefore, the correlation behavior presented by the dynamics of the speckle patterns results in an exponential decreasing curve. The lifetime of the exponential decay is proportional to the diffusion coefficient of particles in the liquid environment. 
To perform DLS measurements, a Zetasizer Nano ZS was used, with a laser source centered at $532 \mathrm{~nm}$, functioning performing size measurements in a range from $0.6 \mathrm{~nm}-6$ microns. To prevent an overload in the detection and distinguish very well each speckle pattern, the detector is conventionally located at $173^{\circ}$ of the light traveling axis.

When a sample containing particles with different sizes is under study, the measurement process should be performed several times to obtain a complete distribution of particle's sizes contained in the sample. By this procedure, the size measurements can be mathematically presented in a graphic containing information of the percentage of studied particles vs their size (see Figure 3.5).

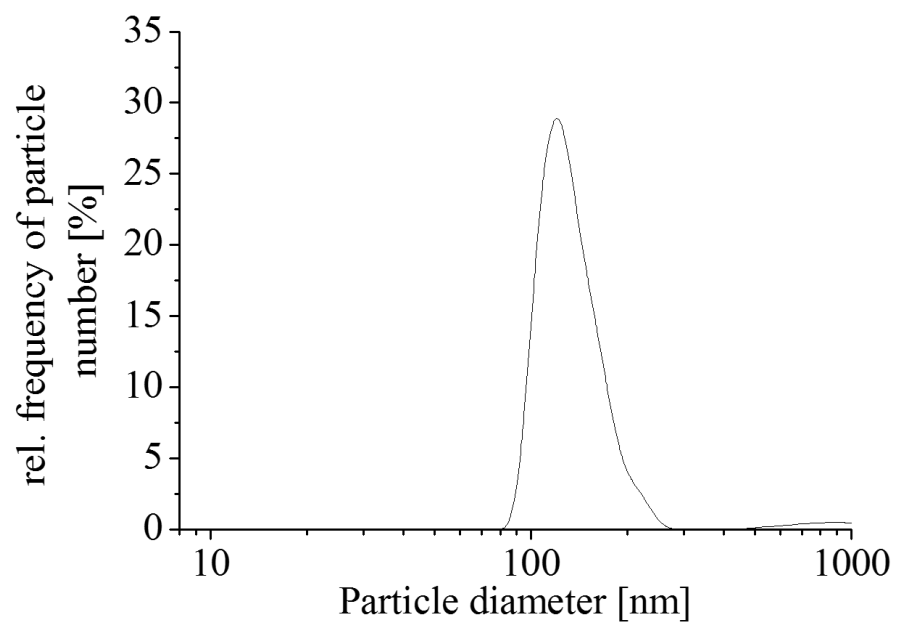

Figure 3.5: Size and size distribution graphic obtained of gold NPs produced during the experiments performed in this research work.

DLS not just enables to measure size and size distribution of nanostructures, it also enables to perform accurate measurements of colloids stability. Traditionally, the stability of colloids can be predicted by knowing the total energy of interaction between two particles. For the size range of colloids containing solid nanoparticles the ratio particle surface to particle volume tend to be so high, and all the interactions are controlled by short-range forces like Van der Waals or electrostatic attraction [143]. As a consequence of the attractive nature of those forces, particles suspended in a liquid environment tend to stick with each other when come into contact due to collisions promoted by Brownian motion. This agglomeration phenomenon leads to sedimentation of the solid material and a modification of properties induced by the spatial confinement. The agglomeration phenomenon can be studied by measuring 
the size of particles in the colloid through the time, making possible to observe the dynamics of particle agglomeration. However, this is not the only way in which the instrument Zetasizer Nano ZS can help us to determine the stability of the samples. The device is also equipped with a pair of cathodes that allows the transmission of electrical current through the sample and therefore, perform electrophoresis. This process may exist due to, the particles that have an electrical charge in their surface and are under the influence of an external electric field, tend to move in the liquid with a constant velocity, not due to the Brownian motion but rather due to the force exerted by the viscosity of the liquid that prevents movement is defeated when an electric field is applied to the sample. As the charged particles tend to go to one of the cathodes oppositely charged, when finally the balance between the attractive force and the viscosity is reached, the particles can move. The velocity of the particles achieved by this experiment is known as electrophoretic mobility, and it is experimentally determined by Laser Doppler Velocimetry (LDV). In LDV a light beam crosses the sample where many particles are moving from one point to another, the scattered light at an angle of $17^{\circ}$ is compared with the incoming beam and this promotes a fluctuation through the time as in the case of DLS. Therefore the rate of the fluctuations is proportional to the velocity of the particles. It can be used to determine the zeta potential of the particles through the Henry's equation [144].

$$
V_{E}=\frac{\varepsilon z}{\eta}
$$

Where $\varepsilon$ represents the dielectric constant of the particle, $\eta$ the viscosity of the medium, and $z$ the $\mathrm{z}$ potential.

The zeta potential of the particles is an electric potential located at the surrounding of the inter-facial region between the particle and surrounding medium. It is generated because the surface's charge distribution of particles induce the increment of ions in the vicinity of the particle. Therefore, the particles are "coated" by an electrically negative layer and a positive one belonging to the environmental medium. The magnitude of this electric potential which belongs to the particles indicates how stable a colloidal suspension can be. If the potential is so large, the particles will tend to repeal to each other making agglomeration phenomenon not probable. Empirically, it has been demonstrated that colloids with zeta potential absolute values above $30 \mathrm{mV}$ tend to be stable [144].

In summary, optical techniques used in this research work helped to determine the size, size distribution, morphology and stability of samples created by PLAL. To prepare the samples, for UV-visible spectrometry, an amount of newly created colloid 
is separated and is poured into a quartz cuvette suitable to be used with a Cary 300 UV-Vis spectrophotometer. For DLS measurements, an amount of the newly created colloid is also separated and pured into a quartz cuvette suitable to be used with the Zetasizer Nano ZS, and also another amount is separated to be pured into a capillary cuvette specially designed for the Zetasizer Nano ZS instrument. The cuvette is equipped wit a couple of cathodes that can be in contact with both the sample and two external cathodes that can transmit an electrical current. The reduction of the sample's thickness that the laser beam should cross is important for LDV experiment since it avoids possible obstructions of the passage of light due to a high concentration of particles.

\subsection{Thermal techniques}

For the first two experiments performed in this research work, thermal properties were specially interesting, due to the mixture of NPs with remarkable thermal properties can promote the modification of thermal properties of the environment in which they are contained. To be more precise, when nanomaterials with a high thermal conductivity or a high capacity to conduct kinetic energy through its forming molecules, is immersed in liquids with a low thermal conductivity, generally liquids composed by huge polymeric chains in which elements present strong chemical bonds and doesn't allow a rapid kinetic energy transfer, then the whole system presents a higher thermal conductivity, due to a collaborative kinetic energy transfer process [145]. This means that nanomaterials could be used to enhance the thermal conductivity of materials with a poor thermal conductivity. If those materials present another interesting properties, the final mixtures result into a new interesting material with more than one remarkable property to be exploited [146].

The conventional way to measure the thermal conductivity in a solid is made by inducing a high temperature in a certain point of the solid material under study, and at at distance $l$, the temperature is measured. The speed with which the temperature of one point is transferred to another, will be directly related to the thermal conductivity of the material. For liquid materials, the traditional method is using coaxial cylinders, the idea is to place the liquid sample between them and induce a high temperature in the inner cylinder and measure the changes in temperature in the outer cylinder, as soon as it starts experiencing a temperature change, the speed at which the temperature travels through the liquid can be measured and thus the thermal conductivity. 
To measure this parameter, we have chosen a highly accurate technique: Hot Wire transient Method. The method is based on the measurement of thermal rise at a certain distance near to a linear source of high temperature (hot wire). The hot wire is immersed in the liquid medium, also a temperature sensor is immersed but located at a certain distance of the heat source. Therefore, the hot wire is heated until it reaches a constant temperature, when the sensor reach a constant measured temperature, the experiment begins [147]. A change in the temperature of the hot wire is induced constantly for a period of time $t$ and the sensor measures the change, which can be described mathematically by the following relation:

$$
\Delta T(l, t)=\frac{q}{4 \pi \kappa} \ln \left(\frac{4 a t}{l^{2} C}\right)
$$

Where $l$ represents the distance between the sensor and the hot wire, $q$ is a constant quantity in which the hot wire produces heat per unit time and per unit length, $\kappa$ is a constant which represents the thermal conductivity, a represents the thermal diffusivity of the tested material and $C=\exp (\gamma)$ with $\gamma=0.5772157$ the Euler's constant.

The thermal diffusivity of the materials is normally represented as follows:

$$
a=\frac{\kappa}{\rho c_{p}}
$$

Where $\rho$ represents the material's density and $c_{p}$ the heat capacity. The formula 3.15 is fulfilled when the distance between the hot wire and the sensor is too short and the experiment is performed at long periods of time. When the temperature difference is plotted vs the natural logarithm of time, the resulting curve is going to be similar to a straight line and in the equation 3.15 .

$$
\frac{q}{4 \pi \kappa}
$$

Will represent the slope of the line, making possible to obtain the thermal conductivity without knowing the density or the heat capacity of the material. Therefore, this technique is suitable to measure the thermal conductivity of colloidal materials created by PLAL.

Finally, it is important to mention that the concentration of particles created by PLAL in different liquid media was performed using an ICP-MS spectrometer quadrupole analyzer Agilent 7500cx equipped with a collision cell. This device allows to determine the amount of chemical elements in a material due to vaporization and atomization of the sample through its exposition of an argon plasma, once the 
ionization of atoms is formed, the ions are separated by means of a quadrupole and directed to the detector, by measuring the respective energies is possible to determine the amount of chemical elements contained in the sample [148].

This sophisticated equipment was used due to its high accuracy level in competition with conventional concentration measuring methods. 


\section{Chapter 4}

\section{Setup Details}

In this chapter, a review of the experimental challenges in this thesis work is given. The experimental details and considerations to be taken into account when using this technique are addressed. Special attention is given to the focusing system, considering that besides of the correct choice of physical and chemical parameters. Experimentally, the biggest control of the operator lies on how the light is driven to the solid target, which is explained on the basis of the manipulation of the laser radiation by optical elements.

\subsection{PLAL and post-irradiation setups}

In all of the experiments performed, a Ti:Sapphire laser (Femtopower Compact Pro, Femtolasers) was used (see Figure 4.1). This laser source is composed by a femtosecond oscillator delivering short pulses with central wavelength of $800 \mathrm{~nm}$ and temporal duration of about $10 \mathrm{fs}$ at a repetition rate of $75 \mathrm{MHz}$ and peak power of about $10 M W$; and Ti:Sapphire femtosecond amplifier delivering $30 \mathrm{fs}$ pulses at a repetition rate of $1 \mathrm{KHz}$ and peak power of about $10 \mathrm{GW}$. The amplifier stage uses the oscillator unit as a seed so that both sources cannot be operated simultaneously. The amplification stage is based on a multi-pass architecture for broad bandwidths, narrow pulses, and elevates the ratio of the main pulse to the amplified spontaneous emission (ASE) background. The system uses prism compressors to avoid chirp and dispersive mirror technology to compensate higher orders of dispersion, and at the same time these elements guarantees an optical transmission of $85 \%-90 \%$.

Recurring measurements of the energy used for each experiment, were performed by using an analogical power-meter (Spectra Physics, Model 407-A) able to withstand $20 \mathrm{~kW} / \mathrm{cm}^{2}$ average continuum wave power density, and with an accuracy capable of measuring few milliwatts. The sensor is able to absorb in a wide spectral range, from 


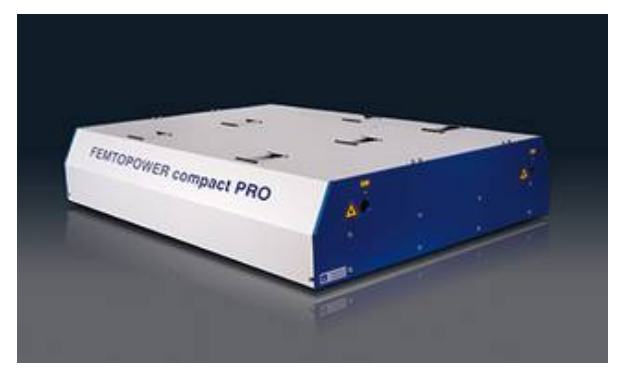

Figure 4.1: Photograph of the laser used for the experiments.

UV to IR, varying by $\pm 1 \%$ between $400 \mathrm{~nm}$ and $1000 \mathrm{~nm}$. Readings are extremely fast since the detector presents a linear response through a BNC output.

The incoming energy was controlled by using a set of calibrated neutral density filters. The cross-section of the profile's beam was slightly elliptical, with a nominal beam diameter of $15 \mathrm{~mm}$ in accordance with the $1 / e^{2}$ criterion. To reduce optical aberrations and increase the spatial uniformity, the beam passes through a $2 x$ allmirror beam expander. In addition, to control the laser light input power, an iris of $6 \mathrm{~mm}$ of diameter is placed before the focusing optics.

For the experiments where PLAL was performed, a classical experimental setup was chosen following the spacial details depicted in Figure 4.2. Therefore, for all the experiments a polished gold disc of thickness $1 \mathrm{~mm}$ and diameter $6.5 \mathrm{~mm}(99.99 \%$ purity) was used as solid target. The gold target was placed at the bottom of a glass vessel (cuvette) filled with the required liquid environment.

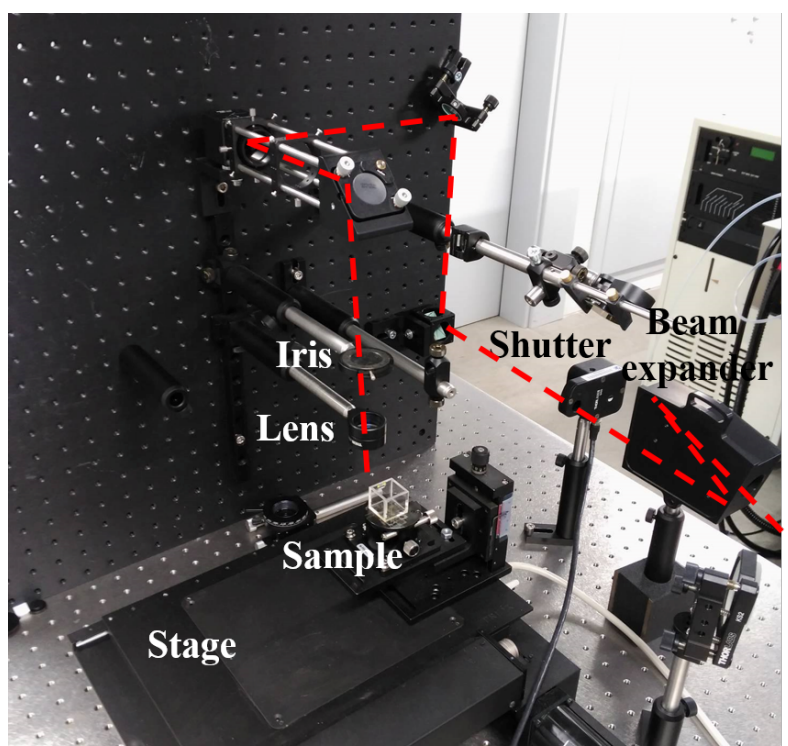

Figure 4.2: Image of the real setup used in the experiments performed, where a dashed line shows the laser radiation path. 
The cuvette was attached to a 2-D motion controlled stage (Prior Scientific, Model H105) with a resolution $<1 \mu m$ and a repeatability of $\pm 0.7 \mu m$, which can be moved at a constant speed during the experiments. The target was irradiated from the air-liquid interface by means of a focused beam with a lens onto the target surface while moving the target (the focal length of the lens was optimized for each experiment), perpendicular to the beam propagation axis in in parallel lines that don't overlap as it is depicted in Figure 4.3, at a constant velocity of $0.45 \mathrm{~mm} / \mathrm{s}$ in each case. The dispersion introduced by the liquid and the optical elements that interact with the laser radiation before reaching the solid target was compensated manipulating the compression prisms of the laser. For the three experiments, the optimized fluence of the laser beam at the sample plane was set to $1 \mathrm{~J} / \mathrm{cm}^{2}$ to avoid plasma breakdown of the liquid, but to have enough energy exceed the ablation threshold for gold $0.5 \mathrm{~J} / \mathrm{cm}^{2}[74,149]$. To adjust the duration of exposure to laser radiation, an electronically controlled shutter was utilized.

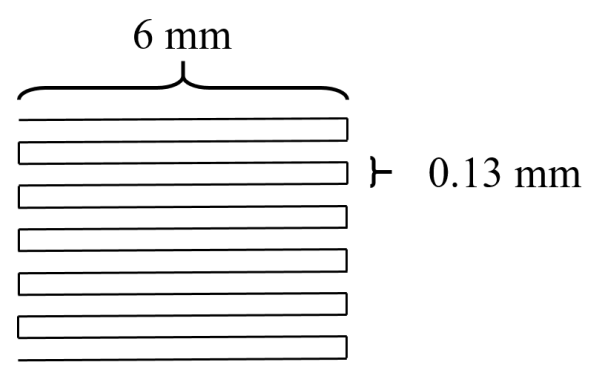

Figure 4.3: Path followed by the 2-D motion controlled stage.

For the experiments where the colloid coming from PLAL experiments was postirradiated, we filled a $10 \mathrm{ml}$ quartz cuvette (Hellma, Model 110-40-40) with the NP colloidal solution obtained from the first experimental stage. The quartz cuvette has a transmission value of about $90 \%$ in the spectral region $200-2500 \mathrm{~nm}$, and a quartz wall that interacts with the incoming beam with a thickness of $1.25 \mathrm{~mm}$. Using this device features, a minimal energy lose and aberration is ensured.

The cuvette is irradiated through one of the quartz walls as illustrated in Figure 4.4, to ensure a minimal optical aberrations and pulse broadening by the interface that the laser beam should cross through its path.

Using the radiation from the Ti:sapphire laser, the beam focused onto the cuvette center with a lens of $75 \mathrm{~mm}$ focal length, while the colloid is stirred by a magnet to homogenize the post fragmentation process. In this process, the laser fluence used is set to $1 \mathrm{~J} / \mathrm{cm}^{2}$. 


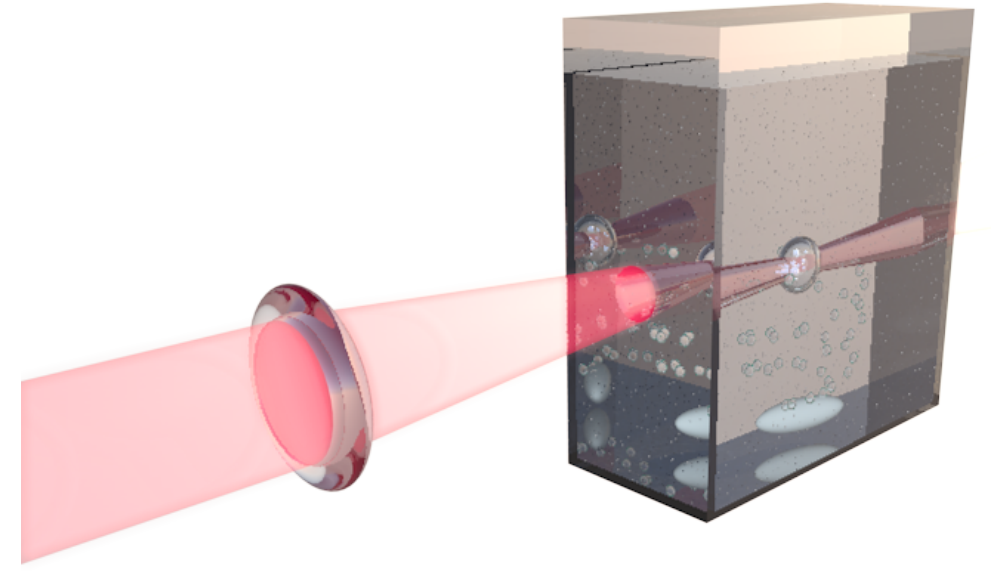

Figure 4.4: Experimental setup used to irradiating the colloids prepared by PLAL.

\subsection{Focusing system}

Fabrication of nanostructures by the use of PLAL is an exciting and young research area where there are plenty of details to discover before it could compete with other nanostructure generation techniques in productivity. Seeking the optimal parameters to achieve these goals, it has been demonstrated that the amount of fabricated material increase proportionally with an increment in the fluence applied [103], but technological limitations prevent obtaining large amounts of material [40]. In particular, one of the most interesting issues is, the determination of the focal plane when high energies are used. Ablation can be promoted out of the focal plane for femtosecond radiation but the maximum ablation efficiency is obtained at the focal plane due to an increase in the peak intensity delivered allowing the detachment of material by either thermal and coulombic mechanisms.

Traditionally, when the focal plane of a beam focused in a solid target in a gas atmosphere needs to be determined, the easiest way is to analyze the ablated spots resulting from different lens-target distance [103]. Nevertheless, when the ablation process is being performed in a liquid ambiance, the determination of the focal plane is more complex.

Considering the conventional experimental setup, the beam path encounters at 
least two interfaces, air-liquid, liquid-solid, and at least two mediums with different optical properties. The strongest influence for any kind of laser source is a shift in the geometrical focal plane due to refraction, normally the shift can be determined using the ABCD-transfer-matrix method for Gaussian beams [150]. Nevertheless, when pulsed laser sources at high intensities are used, linear and nonlinear optical effects start to appear like self-focusing, super-continuum generation or filamentation [151]. In the most of the cases, these phenomena lead to lose of energy resulting in a decrease of ablation efficiency. Another important phenomenon which needs to be considered is the optical breakdown in the liquid environment, which is achieved at high intensities [40]. The optical breakdown is a plasma created in the region where the laser radiation exceeds an intensity threshold, these energies are normally achieved at the focal plane when moderated input power is used. Therefore, no matter the amount of energy used, the right localization of the focal plane in PLAL is mandatory to ensure the best ablation performance and to know how much peak intensity is being used.

To this end, Stephan Barcikowski and co-workers [152] have developed a theoretical approach which helps to determine the focal plane location under conventional PLAL conditions. The approach takes in to account the liquid layer thickness above the target, its refractive index, the amount of peak power used, and based on a modification of the ABCD-transfer-matrix formalism for nonlinear propagation, is possible to determine the radius and therefore the peak power of the laser beam all along the propagation path. The beam radius is expressed by the following analytical set of equations:

$$
\begin{gathered}
\omega(z)=\sqrt{\frac{2}{\kappa}\left|\frac{\sqrt{\frac{P}{P_{c}}-1}}{A}\left[(z-B)^{2}-A^{2}\right]\right|} \\
A=\frac{1}{2} \kappa \omega_{1}^{2} \frac{\sqrt{\frac{P}{P_{c}}-1}}{\left(\frac{P}{P_{c}}-1\right)-\left(\frac{\kappa \omega_{1}^{2}}{2 R_{1}}\right)^{2}} \\
B=\frac{1}{2} \kappa \omega_{1}^{2} \frac{\frac{\kappa \omega_{1}^{2}}{2 R}}{\left(\frac{P}{P_{c}}-1\right)-\left(\frac{\kappa \omega_{1}^{2}}{2 R_{1}}\right)^{2}} \\
P_{c}=\frac{3.77 \lambda^{2}}{8 \pi n_{0} n_{2}}
\end{gathered}
$$

Here, $\kappa$ represents the wave number, $\omega_{1}$ the beam radius at the gas-liquid interface, $R_{1}$ the radius of curvature of the Gaussian wavefront at the air-liquid interface, $n_{0}$ 
the real refractive index of the liquid environment, $n_{2}$ the nonlinear refractive index of the liquid environment, $\lambda$ the laser source wavelength, $P_{c}$ denotes the critical power in which self focusing effects start to appear, $P$ is the incoming laser power, $z$ is a variable which denotes the spacial axis in which the beam is traveling, $\omega(z)$ denotes the beam waist at different $z$ positions, and $R$ is the radius of curvature of the Gaussian wavefront at the $z$ position which can be easily derived from the following equation:

$$
R_{z}=z\left[1+\left(\frac{z_{R}}{z}\right)^{2}\right]
$$

where $z_{R}$ denotes the Rayleigh length.

When the focal plane and the beam radius is finally determined, the peak intensity can be easily estimated by the following equation:

$$
I_{p}=\frac{P_{p}}{2 \pi r^{2}}=\frac{P}{\tau \delta t 2 \pi r^{2}}
$$

Here, $P_{p}$ represents the peak power, $P$ the incoming average power, $r$ the radius of the beam, $\tau$ the repetition rate of the laser pulses, and finally $\delta t$ represents the temporal width of each laser pulse measured in accordance with the $1 / e^{2}$ criterion.

According to this theory, using the experimental characteristics that we propose in this thesis work. A pulsed laser source with a repetition rate of $1 k \mathrm{~Hz}$ would be delivering pulses each millisecond, if the sample is moving at $0.45 \mathrm{~mm} / \mathrm{s}$, the distance among each pulse would be $0.45 \mu \mathrm{m}$. For ensuring the maximum optimization promoting no overlapping between pulses, allowing the radiation to hit always new material's surface, the focal length and layer above the solid target shouldn't exceed $1 \mathrm{~mm}$ and $1 \mu m$ respectively. In this thesis work we didn't avoid overlapping of pulses since the research effort was a lot more focused on the possible applications of the produced materials. Nevertheless, for future research in which the optimization of PLAL experimental setup demands the maximum productivity rate, it would be mandatory to increase the speed at which laser radiation scans the target's surface. In this context, an experimental setup wherein the sample moves could represent problems, since the air-liquid interface would tend to move in the " $\mathrm{z}$ " axis. 


\section{Chapter 5}

\section{Synthesis and characterization of gold/water nanofluids suitable for thermal applications produced by Femtosecond Laser Radiation}

This chapter provides a detailed review of the experiments performed to produce a nanofluid composed by water and gold NPs. The nanofluid was tested for its potential exploitation in thermal applications and harvesting of solar light. This work constitutes the first attempt to synthesize a nanofluid by PLAL during the of the $\mathrm{PhD}$ studies.

The idea of this work, was to manufacture nanofluids which may stand the undergo of drastic temperature changes without suffer damage, by a "green" alternative which ensure no production of chemical pollution and the maximum NPs surface activity which may lead them to be profitable. Since PLAL is a technique based on laser irradiation that promotes the extraction of NPs from almost any kind of solid target in almost any kind of fluid without the use of any external chemical element, PLAL is a synthesis technique that seems to be suitable for the ever-increasing seek for optimization of the existing technologies used to face the problem of energy demand of the modern society. In this context, since solar power concentrators represent a viable alternative for providing energy to a large amount of people, the main concern of this work is to optimize the performance of this device. There are several parts in SPCs that can be improved, but maybe the most important one is the way in which the solar light is actually converted into thermal energy that eventually can be stored and transformed into other kinds of energy. In the present work, we investigate the optical and thermal properties of nanofluids composed of aqueous suspensions of gold NPs manufactured by PLAL, since the technique promise to acquire highly pure and 
well dispersed gold NPs that can be stored at room temperature without showing any significant agglomeration in months. The nanofluids were submitted to thermal cycling and compared to those obtained for commercial gold/water suspensions in view of their use as sunlight absorber fluids in SPCs. The results reveal that the materials manufactured by PLAL don't show any substantial differences for thermal applications with commercial NPs. Therefore, nanofluids produced by this technique can be used in thermal applications which are foreseen for conventional nanofluids, e.g. heat transfer enhancement and solar radiation direct absorption; but giving the opportunity to produce them in-situ in almost any kind of fluid without production of chemical pollution.

\subsection{Introduction}

Fossil fuels are running out, and the search for ways to generate or extract energy increases. Renewable solar energy has demonstrated to be a potential essential tool to keep the ever-increase demand of energy of our society with a minimal environmental impact, since the sun provides an estimate of energy of 120,000 TW every hour [153]. Currently, there are two main technological options to harvest the solar energy and transform it into electricity. Photovoltaic cells (PV) and solar power concentrators (SPC). Considering that the energy should be provided for large amounts of people, SPC seem to be the best cost effective option. SPC concentrates the solar radiation into surface absorbers that convert the majority of the incoming solar radiation into heat, which eventually is transferred to a fluid which can either store that energy or be used to produce steam and activate turbines to generate electricity [154].

However, this energy harvesting approach is not yet cost competitive with the traditional, nonrenewable, fossil energy technologies, mainly due to the efficiency of the system is limited by the operating temperature of the heat transfer fluid, and also because the heat generation at the absorber is separated from the fluid leading to radiative losses. In this context, big research efforts are being conducted to improve the efficiency and reduce the cost of the production and storage of renewable energy as the use of nanomaterials [155]. Maybe the first attempt of using small structures to improve the thermal characteristics of liquids is historically located in 1873 when J.C. Maxwell proposed the addition of small solid particles into a base fluid to increase the thermal conductivity of the suspension [156]. However, the use of millimeteror micrometer- sized particles led to problems such as poor suspension stability and channel clogging, which limits its practical applicability. To solve these drawbacks in 
1995, S.U.S. Choi proposed the use of nanofluids to increase the thermal conductivity of conventional heat transfer fluids [146]. Since the high surface-to-volume ratio and therefore large heat transfer interface between particles and fluid, the high dispersion stability with predominant Brownian motion and the reduced particle clogging make nanofluids suitable for its applicability in heat transfer fluids improvement.

Among the most important physical properties of heat transfer fluids, those which usually are evaluated to determine if are or not suitable for their use in real SPCs are thermal conductivity and heat transfer [157-161]. In this sense, different kind of nanostructures have been investigated, from metal, semiconducting and insulating NPs [162-165] to carbon-based nanostructured complexes [166-168]. These colloidal suspensions have increase the thermal conductivity of the base fluid and in some cases also the heat capacity, even when the NPs concentrations used are so low ( $1 \%$ mass fraction) [169].

However, even when the nanofluid that shows the best thermal performance gets found, is also important to notice that no matter how high thermal conductivity or heat transfer it shows, the fluid would not be used in the moment when it gets the highest temperature, before its final use is going to be moving all along the SPC device loosing energy. A different approach to face this issue, where the heat transfer fluid itself acts also as solar absorber dated back to 1975 and employed India ink [170]. The use of a fluid working both as volumetric light absorber and heat exchanger seems to be advantageous over the classical solution of a transparent fluid exchanging heat with a solid absorber when a nanofluid is being used, mainly due to the remarkable optical properties presented in NPs such as plasmonic effects [171]. Moreover, at low particle concentrations it was also observed steam generation without heating in solution mixtures when the nanofluid is exposed to electromagnetic radiation, allowing applications such as light-induced phase separation in liquids [172].

Thus, the use of nanofluids as direct solar absorbers in either solar collectors and light-induced phase separation had been investigated by several groups [173-178] and with different NPs [138, 179-182], demonstrating that the most important factors influencing nanofluid properties are size, shape and agglomeration state of nanoparticles $[183,184]$. Therefore, a general synthesis technique that promotes a high control over those parameters for a wide range of materials that also fulfils the 12 principles of a "green" chemistry technique is mandatory [185]. The conventional synthesis approaches that promise these results are based on the reduction of complex molecular systems in aqueous mediums, and the size, shape and stability properties are controlled by the use of stabilizers. As it can be inferred, these approach leads to 
production of chemical pollution and in the most of the cases, the use of surfactants can promote further crossed-chemical effects that could modify the nanofluid. Considering the requirements needed for the synthesis approach sought, and challenge that this represents for the scientific community. In this work we propose the use of PLAL to produce nanofluids that will be used for harnessing of solar power. The selection of experimental parameters such as light wavelength, laser fluence, ablation time, repetition rate, or the base fluid itself can modify the shape and size distribution of particles [186]. Moreover, the size and size distribution of particles can be highly modified by a post processing stage based on laser irradiation (PPLI), promoting photo-fragmentation or melting of NPs in the fluid [187, 188]. The whole strategy (PLAL-PPLI) don't require the use of any chemical substance as stabilizer but in contrast promotes the fabrication of metal NPs with electron acceptors at the particle's surface due to atom oxidation $[189,190]$ what produces a relatively high particle surface charge leading to electrostatic repulsion between the particles providing the necessary conditions of stability through the time without significant agglomeration of particles.

In particular, in this research work we produce gold NPs dispersed in water by PLAL-PPLI, and the thermal and optical properties of the fluid are studied and compared to those of available commercial nanofluids containing additives. The morphological characteristics, purity and stability of the materials was determined by Dynamic Light Scattering (DLS), Transmission Electronic Microscopy (TEM) and Energy dispersive X-ray spectroscopy (EDX) tests. The optical properties were analyzed through the measurement of spectral resolved optical absorption and their thermal properties were evaluated by thermal conductivity measurements, carried out by transient hot wire technique. All the samples were submitted to thermal cycles and their properties were evaluated before and after this, to ensure its practical applicability.

\section{$5.2 \quad$ Experimental Setup}

\subsubsection{Material synthesis}

The experimental setup used for this research work is the one depicted in Figure 2.5. The process consists of two stages, in the first one, a pulsed laser beam is focused over the surface of a target producing ablation where the ejected material is captured in a liquid environment consisting of $3 \mathrm{ml}$ of deionized water $8 M \Omega$. The laser beam is focused onto the focusing plane of a gold disc (99.99\% purity Alfa Aser) of diameter 
$6.5 \mathrm{~mm}$ and a thickness of $2 \mathrm{~mm}$, which is placed at the bottom of a glass cuvette filled with water, using a $75 \mathrm{~mm}$ lens, while the beam diameter is $6 \mathrm{~mm}$ at the $1 / \mathrm{e}^{2}$ point considering that the profile's pulse is Gaussian. The cuvette is attached to a 2-D motion controlled stage that is moving at a constant speed of $0.45 \mathrm{~mm} / \mathrm{s}$ during $10.45 \mathrm{~min}$. The thickness of the water layer above the gold disc is about $7 \mathrm{~mm}$, and the fluence used to irradiate the sample is $1 \mathrm{~J} / \mathrm{cm}^{2}$. The fluence is calculated by means of Barcikoski's approach for a pulsed laser beam when is focused and in its path travels through a change of interface air-liquid [152]. Considering that the optical elements and the liquid itself introduce dispersion, a beam post-compression stage based on two fused silica Brewster prisms is used, controlling the dispersion in the beam delivery path.

For the second stage, the colloid obtained from the first stage is irradiated by the pulsed laser beam. The colloid is placed in a quartz cuvette and the laser beam is focused with a $75 \mathrm{~mm}$ lens at a distance of $5 \mathrm{~mm}$ from the interface quartz wallliquid inside the colloid. The energy delivered by the beam in the neighborhood of beam's focus is absorbed by the particles present and as it was described in detail in Chapter 2, the excess of energy absorbed by the particles promotes the instability of the system and the particle tends to explode, forming in this way smaller particles with a low coalescence rate. The process is carried out with a fluence of $1 \mathrm{~J} / \mathrm{cm}^{2}$ calculated for propagation in air while the fluid is constantly stirred with a magnet to homogenize the process. However, considering the propagation in the liquid, the fluence value decreases to $0.6 \mathrm{~J} / \mathrm{cm}^{2}$ approximately ensuring in this way the preference of Coulombian explosion rather than melting of particles since femtosecond pulses are used.

\subsubsection{Preparation of samples}

Nanofluids produced by the PLAL-PPLI technique were compared to commercial nanofluids (Sigma Aldrich) containing gold NPs with $20 \mathrm{~nm}$ nominal primary diameter dispersed in water and either stabilized in 1) $0.1 \mathrm{mM}$ of phosphate buffered saline (PBS) solution or 2) stabilized in citrate buffer solution.

Commercial nanofluids were diluted with distilled water so that the solid content was the same for all samples and equal to the sample obtained by PLAL-PPLI. The gold concentration was measured by inductively coupled plasma mass spectrometry (ICP-MS) and was found to be $15 \mathrm{mg} / \mathrm{l}$. However, it should be noticed that this not assures an exactly equal concentration of NPs among samples, as the NP concentration depends on their sizes and size distribution. All samples were subjected to 
heating-cooling cycles in order to study the influence of the thermal treatment on the properties of NPs and on nanofluid stability. To do this, samples were introduced in a sealed container and heated from room temperature to $100 C^{\circ}$ in a hot bath. After 1 hour heating, nanofluids were cooled down to reach room temperature. A total number of 6 cycles were run for each sample. Table 5.1 lists the investigated samples.

Table 5.1: Samples under study.

\begin{tabular}{cc}
\hline Sample & Details \\
\hline S1 & PLAL-PPLI technique \\
S2 & PLAL-PPLI technique + thermal cycling \\
S3 & PBS stabilized nanofluid \\
S4 & PBS stabilized nanofluid + thermal cycling \\
S5 & Citrate stabilized nanofluid \\
S6 & Citrate stabilized nanofluid + thermal cycling \\
\hline
\end{tabular}

\subsection{Results and Discussion}

To analyze in detail the optical, thermal and morphological characteristics of the materials and determine the importance of using the PLAL-PPLI synthesis approach in the development of nanofluids with potential use in the harvesting of solar light industry, it was first necessary to determine if the proposed method promotes the fabrication of highly pure NPs without the use of any external chemical additive. To this end, is mandatory to recognize if the particles show any evidence of chemical reactions between the particle and the base fluid by showing the appearance of elements that don't belong to the solid target or the base fluid. The techniques used to this end were (EDX and TEM).

From Chapter 4, where the sample preparation for the visualization in TEM is explained in detail, is clear that just solid content is under study. Furthermore the vacuum conditions at which TEM's measurements are performed, only the particles and any possible by-product could be visualized. In Figure 5.1, TEM representative micrographs of the six samples are depicted. From a statistical qualitative analysis, there is no any evidence of solid material that can be considered as by-product neither before nor after the thermal cycling. Apparently it is only possible the visualization of gold NPs.

To ratify these observations an elemental analysis of samples was performed. If any chemical reaction existed between the particles and the fluid, it would be evidenced 


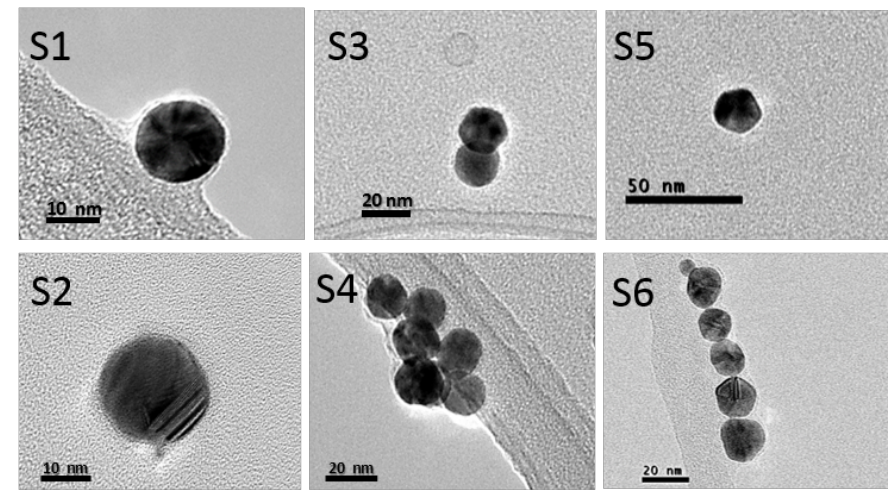

Figure 5.1: TEM micrographs of the samples before and after the thermal cycles.

by the measurement of by-products. In Figure 2, we can appreciate the EDX spectra of the sample synthesized by PLAL-PPLI, the commercial sample stabilized with PBS, and the commercial sample stabilized with citrate. In all of the cases the presence of copper $(\mathrm{Cu})$ and carbon $(\mathrm{C})$ has been detected and it is connected to the composition of the microscope grid. It can be observed that, even if gold ( $\mathrm{Au}$ ) has been correctly detected in each sample, only for PLAL-PPLI nanofluids no other contaminants are present. In fact, commercial samples, stabilized with surfactants also show the elements contained in their dispersant or chemicals coming from the synthesis production process itself, such as sodium (Na).

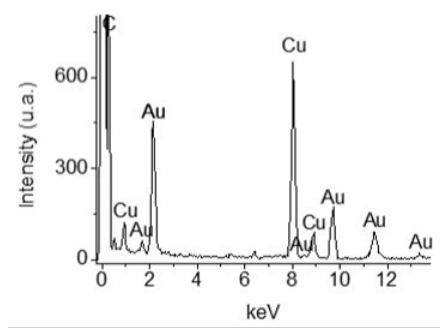

a)

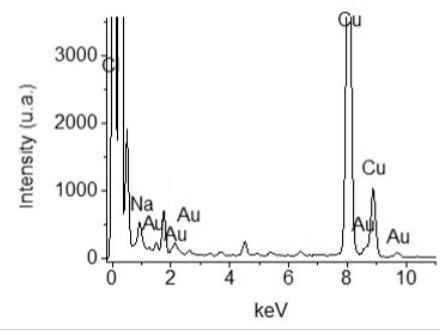

b)

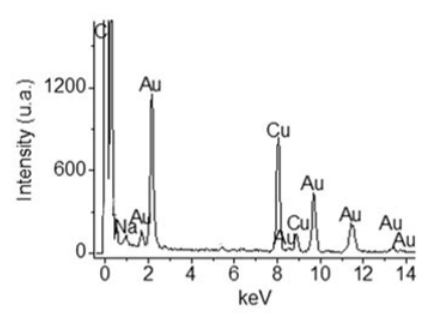

c)

Figure 5.2: EDX spectrum of a) PLAL-PPLI produced NPs (S1), b) commercial NPs in PBS (S3) and c) commercial NPs in citrate buffer (S5).

In the other hand, from a statistical analysis, it was determined that particles created by the laser-based synthesis proposed show the most spherical shape. A possible reason to acquire this shape is that during the creation of particles, the particles undergo a surface tension effect resulting from its interaction with the liquid ambiance, and as in the case of burbles in water, the most stable shape for the particles to acquire is the spherical one. Contrary to this, when particles are created 
by chemical methods, the natural process of particle creation is nucleation of reduced elements and further growth, under the presence of stabilizers or additives, the growth is not promoted in an homogeneous way all over the particle's surface resulting in the creation of particles with non necessarily spherical shapes.

For practical purposes, a non-spherical shape in a metal NP could lead to poor optical response or in nanofluids, it could lead to issues in the flow dynamics. After the thermal treatment, the all of the samples does not show any morphological change or change in size. In particular, the size of particles can not be inferred from the TEM micrographs, since the amount of solid material in the sample is so low and the particles tend to be well dispersed. To assess a correct size distribution, it was mandatory to use DLS.

This technique as it was mentioned in Chapter 4, provides the size distribution of the particles or agglomerates of particles as they are present in the nanofluid in static conditions. The results depicted in Figure 5.3. show the mean particle size, $d_{p 50}$, which is defined as the size below the $50 \%$ of particles that are comprised and is very close to the diameter on the peak of the distribution.

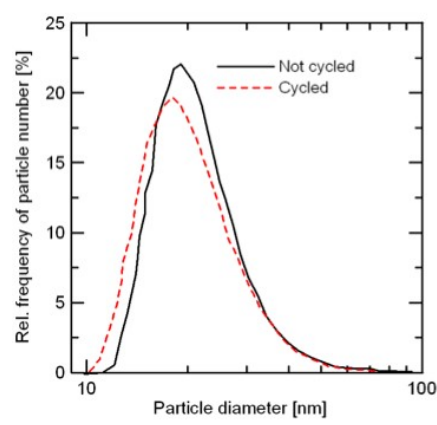

a)

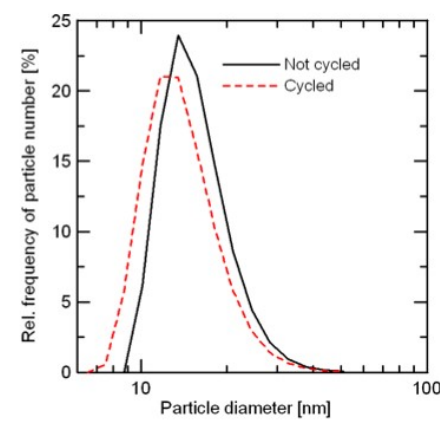

b)

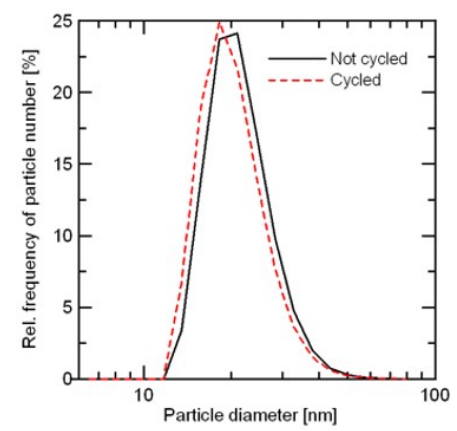

c)

Figure 5.3: Particle size distribution a) NPs produced by PLAL, b) commercial NPs stabilized in PBS and c) commercial NPs stabilized in citrate buffer.

Results are shown in Table 5.2. It can be observed that NP primary average size for commercial PBS and citrate buffer nanofluids is $15.4 \mathrm{~nm}$ and $21.3 \mathrm{~nm}$, respectively. In all the cases, the NP distribution is considerably narrow, with standard deviation values lower than $5 \mathrm{~nm}$. And finally, for the all of the samples, NPs are very well dispersed with almost no clustering after the thermal cycles. As figure of merit, is possible to consider that the clustering can be evidenced when particle sizes are larger than $2 d_{p 50}$, since this value will show the tendency of particles to agglomerate. 
Table 5.2: Mean particle size of nanofluids.

\begin{tabular}{ccc}
\hline & $d_{p 50}(\mathrm{~nm})$ & \\
\hline Sample & Not cycled & Cycled \\
\hline PLAL-PPLI technique & 18.5 & 17.4 \\
PBS stabilized nanofluid & 15.4 & 14.1 \\
Citrate stabilized nanofluid & 21.3 & 20.3 \\
\hline
\end{tabular}

The optical properties in the other hand, determined by measuring the transmittance spectra at room temperature, were assessed comparing both, the produced $\mathrm{Au}$ NPs and commercial Au-NPs in aqueous suspensions. The transmission spectra were acquired with respect to the base fluid and air reference, respectively. In Figure 5.4 we show the overall transmittance spectra of the as-prepared samples with respect to air, corrected for the reflectance term. The curves depicted represent the spectral transmittance of the whole system built by NPs and base fluid.

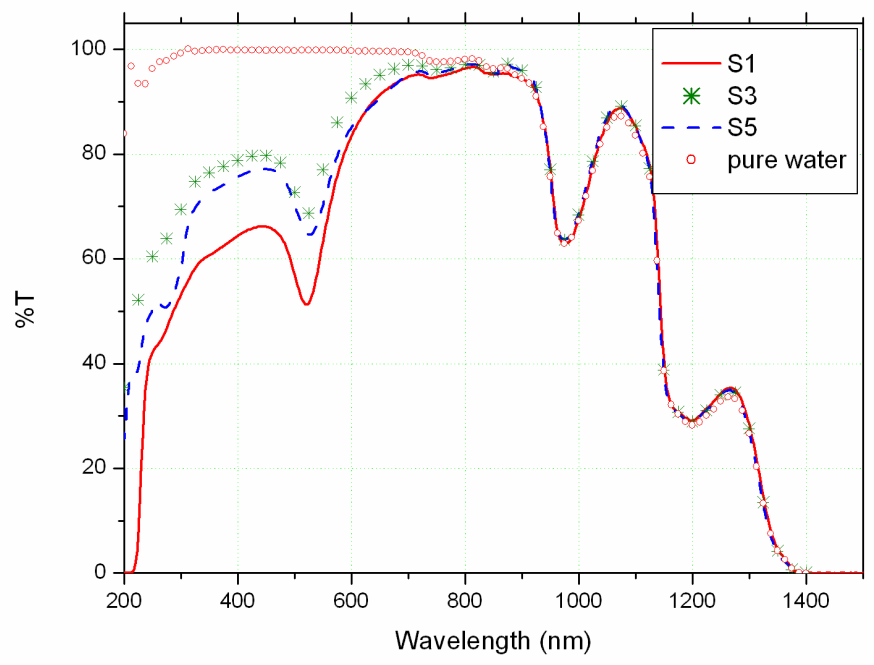

Figure 5.4: Transmittance spectra of as-produced samples. The spectrum of pure water is also shown for reference (red dots).

We can appreciate that the spectra show the surface plasmonic peak, corresponding to a minimum in the transmittance spectrum, in a similar spectral position i.e. at $522.5 \pm 2.5 \mathrm{~nm}$, in agreement with the rough similarity of NP dimensions in the various samples detected by DLS measurements. The difference in the transmittance absolute values of samples near the plasmonic peak could be due to differences in the molar concentration of different NPs sizes within each poly-dispersion. In fact, as 
reported in the literature, both molar concentrations and dimensions of NPs strongly affect optical spectra [56,191-195] and poly-disperse suspensions show an inhomogeneous broadening in the spectra [56]. If we compare, for each preparation technique, the pre- and post-cycling spectra (Figure 5.5) we can notice that PLAL-PPLI and PBS samples practically show no changes, while for citrate samples (S5-S6) the postcycling transmittance is slightly decreased, likely because of some degradation of the citrate additive.

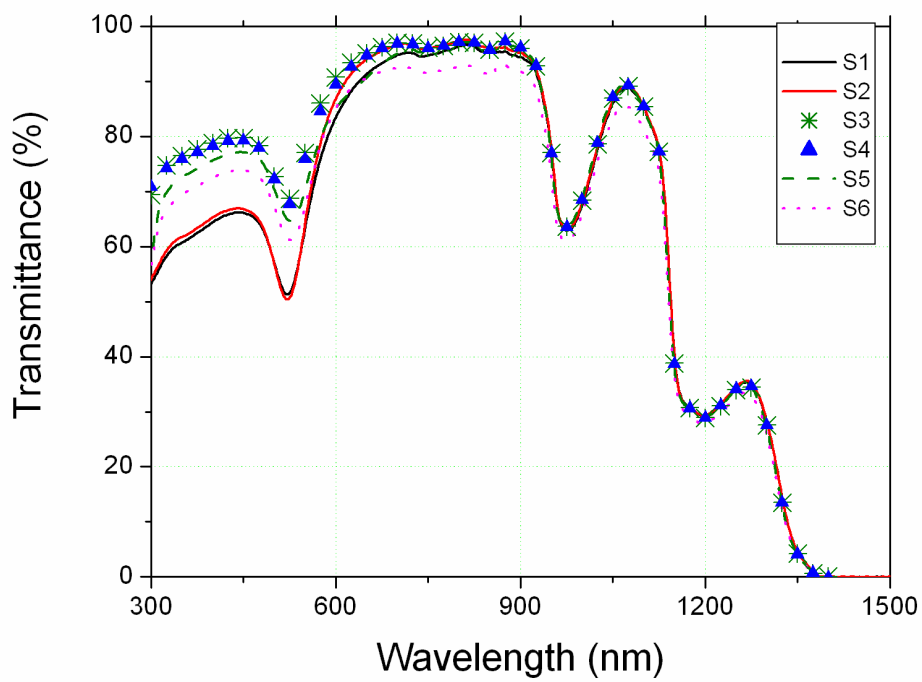

Figure 5.5: Comparison of transmittance spectra of as-produced and cycled samples.

To decouple the contribution to light extinction due to NPs and base fluid, respectively, we acquired the transmission spectra using a cuvette filled with distilled water as reference. Figure 5.6 shows the calculated extinction coefficient of as-produced samples. Again, the surface plasmonic peak (transverse surface plasmon resonance) can be clearly recognized, as well as a secondary small shoulder at wavelengths below $400 \mathrm{~nm}$, in agreement with the literature [196].

The small features at wavelengths around 965 and $1157 \mathrm{~nm}$ are probably due to instrumental artifacts, as the contingent presence of non-spherical particles exhibiting longitudinal surface plasmon resonances in this spectral region could be ruled out by TEM analyses [197]. The difference in the absolute value of extinction coefficient for the samples can be ascribed to the different size distribution, and thus NPs concentration distribution, as mentioned above.

The extinction coefficient $\mu_{\text {ext }}(\lambda)$ is the sum of the absorption and scattering coefficients. Thus, to give an estimation of the absorption properties of the nanofluid, 


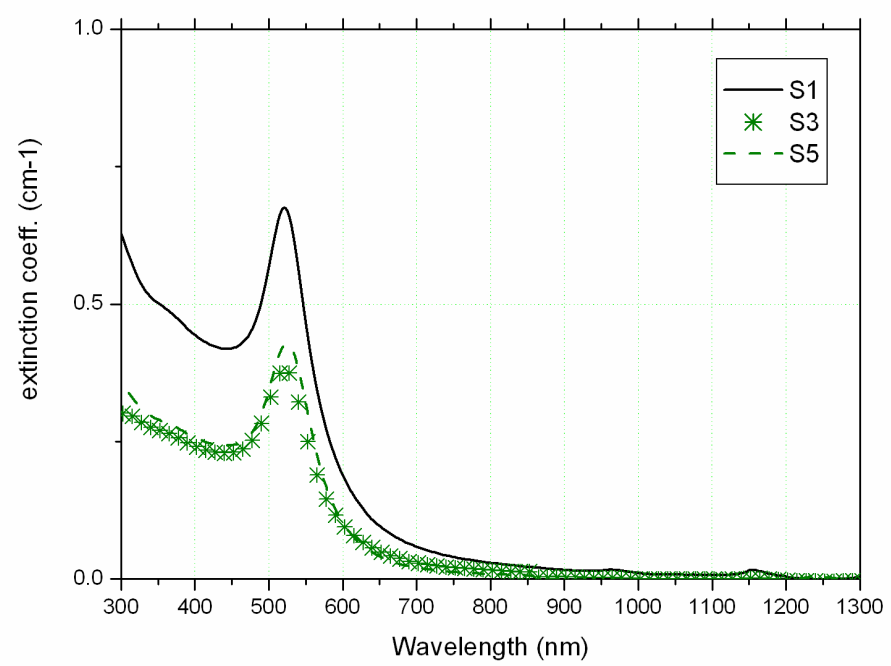

Figure 5.6: Extinction coefficient of NPs decoupled from the base fluid contribution.

it is necessary to evaluate the light scattering characteristics as well. The spectral scattering albedo $\omega(\lambda)$ is defined as the ratio between scattering and extinction coefficients and can be obtained from the calculated extinction and scattering efficiencies $Q_{\text {ext }}$ and $Q_{\text {sca }}$, respectively. Following the notation in [198], are given by:

$$
\begin{gathered}
Q_{e x t}=4 x \operatorname{Im}\left\{\frac{m^{2}-1}{m^{2}+2}\left[1+\frac{x^{2}}{15}\left(\frac{m^{2}-1}{m^{2}+2}\right) \frac{m^{4}+27 m^{2}+38}{2 m^{2}+3}\right]\right\}+\frac{8}{3} x^{4} R e\left\{\left(\frac{m^{2}-1}{m^{2}+2}\right)^{2}\right\} \\
Q_{\text {sca }}=\frac{8}{3} x^{4}\left|\frac{m^{2}-1}{m^{2}+2}\right|^{2}
\end{gathered}
$$

Where $m$ is the complex relative refractive index.

$$
m=\frac{n_{p}+i k_{p}}{n_{f}+i k_{f}}
$$

defined in terms of the real $\left(n_{p}, n_{f}\right)$ and imaginary parts $\left(k_{p}, k_{f}\right)$ of the complex refractive indexes of particles and fluid, respectively; and $x$ is the particle size parameter:

$$
x=\frac{2 \pi n_{f} a}{\lambda}
$$


being $a$ the particle radius and $\lambda$ the light wavelength in vacuum. In the Rayleigh regime $|m| x<<1$, the expression in brackets in Eq. 5.1 is approximately unity. The extinction efficiency thus becomes:

$$
Q_{e x t}=4 x \operatorname{Im}\left\{\frac{m^{2}-1}{m^{2}+2}\right\}+\frac{8}{3} x^{4} R e\left\{\left(\frac{m^{2}-1}{m^{2}+2}\right)^{2}\right\}
$$

For poly-dispersed particles, the scattering albedo is given by [198]:

$$
\omega=\frac{\sum_{i} N_{i} Q_{s c a, i} \pi a_{i}^{2}}{\sum_{i} N_{i} Q_{e x t, i} \pi a_{i}^{2}}
$$

where $N_{i}, Q_{s c a, i}$, and $Q_{e x t, i}$ are the volume concentration, the scattering efficiency and the extinction efficiency, respectively, of the spheres of radius $a_{i}$. From Eq. 5.6 we calculated the single-scattering albedo for our samples in the Rayleigh regime. The complex refractive indexes of water and gold considered in the calculation were taken from [199,200]. For simplicity, the experimental NPs size distributions (Figure 5.3) have been approximated as trimodal dispersions, considering, for each sample, the measured populations for three particle sizes around the peak of the distribution and including at least $85 \%$ of the total number of particles. It should be noticed that the Rayleigh hypothesis is not satisfied for wavelengths in the range about $300-350 \mathrm{~nm}$ for the largest particles in the distribution (which represent about 30\%, 20\% and 5\% of the considered population in the samples S1, S5 and S3, respectively). Thus, in this spectral region the scattering albedo is probably underestimated. The spectral scattering albedo $\omega(\lambda)$ was used to obtain the spectral absorption coefficient $\mu_{a b s}(\lambda)$ of the nanofluid from the experimental extinction coefficient $\mu_{\text {ext }}(\lambda)$ as:

$$
\mu_{a b s}(\lambda)=\mu_{e x t}(\lambda)[1-\omega(\lambda)]
$$

To quantitatively evaluate the sunlight absorption capability of samples for the proposed solar collector application, we calculated the fraction $F$ of the incident power absorbed in the fluid after a propagation length $l$ in it:

$$
F(\lambda)=1-\frac{\int_{\lambda_{\min }}^{\lambda_{\max }} I(\lambda) \cdot e^{-\mu_{a b s}(\lambda) l} d \lambda}{\int_{\lambda_{\min }}^{\lambda_{\max }} I(\lambda) d \lambda}
$$

where $I(\lambda)$ is the spectral distribution of the incident irradiance integrated in the wavelength range $\left(\lambda_{\min }, \lambda_{\max }\right)$. For a still and cold isotropic medium, $\mu_{a b s}(\lambda)$ is considered constant along $l$. In Figure 5.7 we compare the calculated absorbed light fraction as a function of the propagation depth within the fluid, for pure water and 
for the as-produced gold nanofluids. The calculation has been performed according to equation 5.8, keeping as $I(\lambda)$ the CIE solar spectrum with air mass $m=1.5$ [201] and considering $\lambda_{\min }=300 \mathrm{~nm}$ and $\lambda_{\max }=2300 \mathrm{~nm}$. We can observe that even with the low concentrations we have considered, a nearly $80 \%$ absorption can be obtained after a $10 \mathrm{~cm}$ propagation length in the nanofluid. This value is nearly twice than that obtained for simple water. The samples show small absorption differences due to the differences in their extinction coefficient mentioned above. A larger absorption can be obtained with more concentrated suspensions. The results shown demonstrate that gold NP-based nanofluids can be used as volume absorbers in direct absorption solar collectors. Moreover, they offer also the opportunity to optimize the sunlight absorption for the specific solar collector architecture of interest by changing concentration and sizes of NPs.

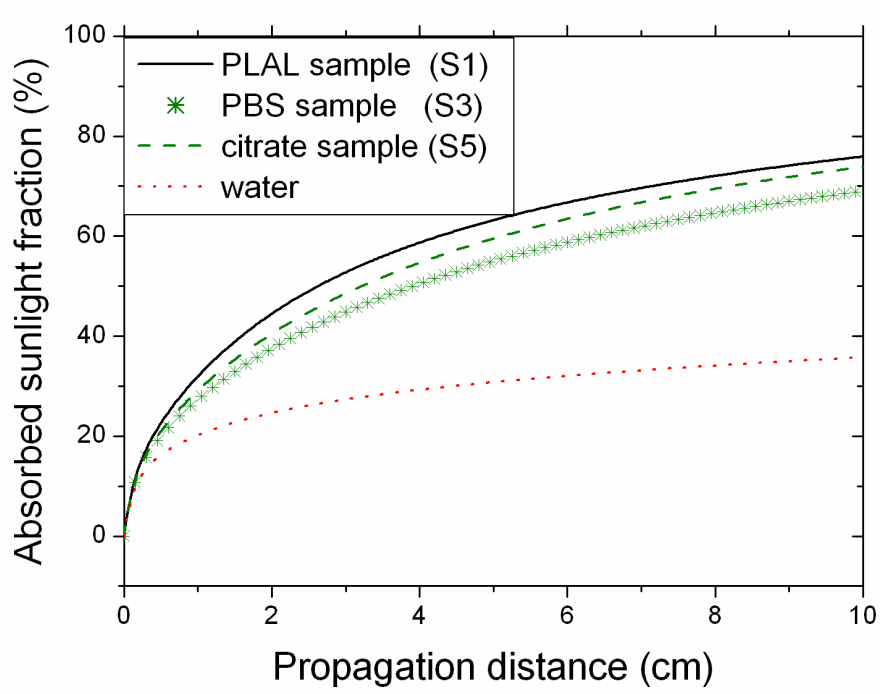

Figure 5.7: Absorbed sunlight fraction as a function of the propagation distance in the nanofluid for the as-produced samples.

In the other hand, as long as the scope of the nanofluid synthesis considers the production of materials with potential applications not just as volumetric absorbers, but also heat transfer fluids. The thermal conductivity of nanofluids produced by PLAL-PPLI and commercial nanofluids was measured at $80 C^{\circ}$ by means of a KD2 Pro conductimeter (Decagon Devices Inc.). The KD2 Pro is the commercial device that measures the thermal conductivity with the help of the transient hot wire technique measuring the temperature/time response of the wire to an abrupt electrical pulse. 
Six measurements were performed for each sample. The experimental error could be determined at $95 \%$ of confidence level.

Table 5.3: Thermal conductivity measurements at $80 C^{\circ}$.

\begin{tabular}{ccccc}
\hline & $k\left(W / m K^{\circ}\right)$ & \multicolumn{3}{c}{$\Delta k(\%)$} \\
\hline Sample & Not cycled & Cycled & Not cycled & Cycled \\
\hline Water & $0.652 \pm 0.013$ & - & - & - \\
PLAL-PPLI technique & $0.667 \pm 0.018$ & $0.666 \pm 0.020$ & 2.23 & 2.20 \\
PBS stabilized nanofluid & $0.687 \pm 0.022$ & $0.677 \pm 0.010$ & 5.42 & 3.88 \\
Citrate stabilized nanofluid & $0.681 \pm 0.009$ & $0.669 \pm 0.013$ & 4.36 & 2.53 \\
\hline
\end{tabular}

From the results represented in Table 5.3, it can be observed that the thermal conductivity of the base fluid increases when NPs are present in the all of the samples. Differences between PLAL-PPLI and commercial nanofluids, as well as before and after cycling lie on the basis of the experimental uncertainty. Therefore, it can be concluded that PLAL-PPLI synthesized nanofluids provide an increase in thermal conductivity similar to commercial suspensions and stable with thermal cycling. As a final comment, it should be noticed that the nanofluids that we have investigated have a very low particle concentration. A larger thermal conductivity enhancement can be obtained with more concentrated suspensions [202]. However, as the NP concentration increases, nanofluids could show stability problems, that in case of chemically-stabilized samples it means the need of a larger surfactant amounts with a detrimental effect on thermal conductivity properties [203]. In this framework, physical techniques like PLAL-PPLI, which are able to produce stable nanofluids without the use of surfactants, can have an even larger impact on solar nanofluids fabrication.

\subsection{Conclusions}

In summary, the most important contributions of this research work lies on the production of gold NPs-based aqueous nanofluids by means of the PLAL-PPLI technique without the use of chemical additives. The produced nanofluid in comparison with commercial available nanofluids shows similar optical and thermal properties before and after thermal cycling and in some cases even better for their potential use in the harvesting of energy industry, e.g. a higher sphericity of suspended particles which could lead to a better nanofluid motion or the purity of the particles. In the 
all of the cases, the nanofluids demonstrated to be able to work under thermal cycling. From the measured extinction coefficient and the calculated spectral scattering albedo, sunlight absorption characteristics have demonstrated that gold-NPs nanofluids have a great potential to be used as direct absorbers in solar collectors. In this context, it should be noticed that optical absorption can be tuned by changing NPs concentration and size. Moreover, it is known that thermal conductivity of nanofluids increases with the NPs volume load. Thus, the advantage of using PLAL-PPLI synthesis approach, which produces stable nanofluids without chemical surfactants can be even larger when high NPs concentrations are required. Representing a synthesis alternative that may inspire future research in the field. 


\section{Chapter 6}

\section{Fabrication of gold nanoparticles in Therminol VP-1 by laser ablation and fragmentation with fs pulses}

This chapter provides a detailed review of the first and second published papers as a consequence of the research activity during the $\mathrm{PhD}$ studies.

Based on the nanofluid idea presented in the last Chapter, the goal for this work was to move a step forward using PLAL to improve the thermal conductivity of an euthectic mixture of diphenyl oxide and biphenyl, commercially known as Therminol VP-1 by fabricating gold NPs in it. The motivation behind this research activity lies on a simple idea: the fluid used in the present work is commonly employed in the harnessing of solar power industry and its thermal improvement could represent a great technological advantage.

Traditionally, in SPCs the fluid used in this research work is heated by its exposure with concentrated solar radiation, the most common type of solar thermal collector utilizes a black surface as absorber element, which transfers heat to the fluid running in tubes embedded within the surface. Then, a heat exchanger transfers the heat of the fluid to a steam turbine system connected to an electrical power generator [204]. Alternatively, in direct absorption solar collectors the solar radiation is directly absorbed by the fluid and transformed into heat and then evaporated to move mechanical engine and finally transform the collected solar energy into electrical and useful energy [138,175,205-207]. Nevertheless, this particular fluid presents a low thermal conductivity and consequently, the time in which the whole fluid can be heated until it's maximum limit could not fulfill the productivity needs of the industry, therefore is mandatory to improve this special characteristic.

Based on nanofluids idea, Wang and co-workers have introduced gold NPs in the 
thermal fluid studied in this work, and it was proved that the thermal conductivity can be enhanced [208], nonetheless their material presented a poor stability and the creation of byproducts. Therefore, to improve the stability and purity of the final material, we have produced gold NPs by PLAL considering that this fabrication technique is an approach in which there is no chemical pollution, making easier to determine the stability of the material since there can not happen any uncontrolled further chemical reactions.

The application of the idea led us to observe a mitigation in the agglomeration of particles through the time, making the final material more stable but keeping a thermal conductivity enhancement of about $4 \%$. Nevertheless, since the scope of the possible applications lies on the harvesting of energy and its possible use in real solar power applications, the two possible further experimental paths focuses on increasing the thermal conductivity or improving the stability of the nanofluid. From previous works, creating metal NPs in the heat transfer fluid used in this work, it is clear that even when both characteristics are strongly desired, the natural tendency of the nanofluid is to do not present both of them together optimized. Nonetheless, preliminarily results let us understand that when the nanostructure in the fluid is smaller, bigger is the surface to volume ratio and stronger is the influence of the presence of NPs in the fluid allowing to obtain bigger thermal conductivity values. And in the other hand, it was also possible to determine that when the particles are smaller, there is more chance to get a stable nanofluid which will end up creating clusters of particles not big enough to settle. Therefore the biggest goal to achieve was to get the smallest NPs size possible to obtain the best thermal conductivity response and the most stable behavior.

During the research work presented here, a set of experiments were performed to determine if the optimization of laser parameters could help to achieve the desired results. And contrary to the hypothesis, the minimization of particle's size didn't help to mitigate the agglomeration phenomenon leading to the worsening of the material. Therefore, PLAL is effective to achieve small primary NPs sizes but maybe not so effective to ensure no agglomeration through the time. Considering the classical Derjaguin-Landau-Verwey-Overbeek (DLVO) theory, which describes mathematically the aggregation of aqueous dispersions based on electrostatic and Van der Walls attraction between the particles, we realized that in an organic oil such as Therminol VP-1 which doesn't promote that the gold particles acquire electric charge, there is a decrement of electrostatic repulsion between particles leading to their agglomeration. 
Then, to mitigate the agglomeration phenomenon we have thought about generating repulsion between particles through steric or electro-steric forces. Steric repulsion is a phenomenon in which molecules with low reactivity or negatively charged envelope another molecular systems to promote repulsion between them and not allowing them to clump. In this context, it was important to find the molecule which promote the steric repulsion in organic oils, but able to resist elevated temperatures. Working temperatures for solar power applications are around $300^{\circ}$. Once the molecule was identified, the next step was to promote the envelope of nanostructures. Using conventional methods the functionalization of particles inside organic oils is complex and require difficult procedures. Nevertheless, the great versatility of PLAL allows the functionalization of NPs by just adding the functional groups to the liquid or colloidal environment as it was explained in Section 2.2.2.1. Therefore, after the functionalization, the result achieved in this research work lead us to obtain a material with a primary NPs size of $58 \pm 31 \mathrm{~nm}$ and reaching a final cluster size of $370 \mathrm{~nm}$ for up to 1 month and keeping the thermal conductivity enhancement.

The application of the idea proposed to improve the novel nanofluid was successful because we have obtained the smallest agglomeration rate and size reported in the literature for Therminol VP-1 and gold, and the thermal enhancement was kept. Nonetheless, the thermal conductivity enhancement did not get a great improvement as the stability, making this result a study that helps to mitigate the stability of nanofluids based on organic oils, and may inspire future research to also improve the thermal conductivity and finally get a definitive material with outstanding properties that could change the way in which solar energy is harvested.

\subsection{Introduction}

As it was discussed in detail in the past chapters, PLAL is a synthesis strategy that has gained the trust of the scientific community through the time due to its versatility and reproducibility. Recently, the right selection of the liquid environment in which NPS should be created has been leading to new interesting results, such as the development of high speed and frequency electronic devices [209], the synthesis of non-toxic NPs that present infrared emission for biomedical applications [210,211], or the creation of new materials that are able to absorb solar radiation in a wide spectral range and transform it into thermal energy, which subsequently can be transformed into useful energy. 
Since one of the fields that demand bigger research efforts is the energy industry, due to an ever increasing demand from the modern society. Several groups worldwide have been testing the idea of introducing metallic particles in different fluids, that could lead to an efficient harnessing of solar power.

At the moment, the best performance for a real application based on nanofluids was presented by Wang et. al. [208] through a bottom-up fabrication approach, whom pointed out the necessity to implement this ideas in daily used commercial fluids to improve its performance in application were are commonly used. In this context, they have demonstrated that the fabrication of spherical gold NPs with sizes around $121 \mathrm{~nm}$ and concentrations of $0.05 \%$ (mass percentage) in Therminol VP-1 can enhance its thermal conductivity by up to $6.5 \%$, thus increasing the harvesting of energy in devices like SPCs. Therminol VP-1 as other eutectic mixtures of biphenyl and diphenyl oxide are heat transfer fluids or thermal oils commonly used for solar power applications, they are used to transform the incoming electromagnetic radiation into thermal energy, afterwards are transformed into steam and the kinetic energy of the vapour particles is used to move mechanical engines and eventually the energy is transformed into electricity $[175,208]$. Since, improvement in the harvesting of solar light is a challenge. One way to increase the efficiency in devices that are used to harvest solar power is by fabricating heat transfer nanofluids with remarkable thermal characteristics, using conventional thermal oils. The manufacture of thermal nanofluids using commercial heat transfer fluids is already possible and are two wellknown methods. In the first one, NPs are first produced by chemically $[212,213]$ or physically [189] routes, and subsequently dispersed into the proper fluid. But as NPs have a high surface energy, aggregation and clustering are unavoidable and will appear quickly. An alternative procedure to prevent agglomeration as much as possible is to produce the NPs directly in the base fluid. However, the typical methods used for this aim such as chemical reduction [214], photochemical reduction [215], glow discharge [216], and sonochemical reduction [217], are not so effective and tend to present an increment of viscosity which is considered as a drawback due to the associated increment in fluid pumping costs, mainly due to pollution and by-products creation during the synthesis, and also present stability problems. And as it is well known, one of the most important characteristics of nanofluids is to know if they can remain for very prolonged periods of time without significant settling or loss of stability.

Hence, an idea to mitigate this unwanted effects is the use of PLAL and a further post processing stage based on laser irradiation (PPLI) due to the NPs can be syn- 
thesized in-situ and highly control its final characteristics. Nevertheless, the use of such pure technique don't ensure the total mitigation of agglomeration. Apart from the production process, a chemical stabilization is required to enhance the stability against agglomeration of NPs. Stability in nanofluids can be predicted by knowing the total energy of interaction between two particles. In the range of nanoscale, the ratio particle surface to particle volume is so high that all the interactions are controlled by short-range forces like Van der Waals attraction force. As a consequence of the attractive nature of the forces presented, when primary particles are suspended in a fluid they have a tendency to agglomerate when they collide due to their Brownian motion. This agglomeration can be avoided by means of repulsive surface forces created among particles. The classical DLVO theory only takes into account electrostatic repulsion, however, in non aqueous media steric or electrosteric repulsion is preferred [186]. It is achieved by the covering of particles with ionic surfactants that generates a charged medium surrounding the polymeric chains adsorbed to the particle surface, thus preventing contact and agglomeration of particles.

In this research work, we experimentally demonstrated the fabrication of a nanofluid, based on a commercial heat transfer fluid and pure gold, by PLAL performed with a femtosecond laser source and post-processing the material by a post-irradiation process, implemented to control the final size of the created nanomaterial. And in the other hand, gold NPs highly dispersed in a commercial heat transfer fluid were produced by PLAL and the PPLI stage with the help of a cationic surfactant previously introduced in the base fluid to stabilize the material. As the separation of the NPs from the surfactant tends to be difficult, it is important that the surfactant is compatible with the end-use procedure. In our case, the surfactant used was tetra- $n$-octylammonium bromide $\left(\left(\mathrm{C}_{8} \mathrm{H}_{17}\right)_{4} \mathrm{NBr}\right.$, TOAB, $\left.>98 \%\right)$ because this kind of dispersant has demonstrated its chemical compatibility with the thermal oil and the solid target and it can be used for practical applications, as TOAB does not degrade at high temperatures.

The morphology, purity, mean size, and size dispersion of NPs, the stability and thermal conductivity enhancement of the nanofluid were determined in both cases. The obtained materials presented an enhancement of thermal conductivity of the base fluid and a considerable improvement over the stability of the nanofluid for the first published results and the highest stability reported for the second published paper. We believe that the findings of this project could have a potential impact on the fabrication of nanofluids based on exotic or chemically complex liquids, giving the 
opportunity to synthesise nanofluids with outstanding properties, not easily achieved by chemical approaches.

\subsection{Experimental setup}

\subsubsection{Material synthesis}

The experiments performed can be summarized into two protocols, each one divided into two stages of a single synthesis route, a colloid manufacture or PLAL stage followed by a post-processing and NPs size control or photo-fragmentation stage without the use of TOAB surfactant and with the use of TOAB. The main difference in the two protocols is the use of TOAB, in the first one there was no any surfactant or extra additive, in the second protocol $0.0547 \mathrm{~g}$ of TOAB was dissolved in $100 \mathrm{ml}$ of the synthetic thermal oil. The rest of the procedure is the same. During PLAL stage, a gold disc of thickness $1 \mathrm{~mm}$ and diameter $6.5 \mathrm{~mm}$ (99.99\% purity) was used as the solid target. The disc was placed at the bottom of a glass cuvette filled with the fluid (Therminol VP-1 for the first protocol and Therminol VP-1+TOAB for the second protocol). The thickness of the liquid layer above the target was about $7 \mathrm{~mm}$. The cuvette was attached to the 2-D motion controlled platform moving at a constant velocity of $0.45 \mathrm{~mm} / \mathrm{s}$. The sample was irradiated from the air-liquid interface by a focused beam with a lens of $75 \mathrm{~mm}$ focal length onto the solid target surface as it can be inferred from the experimental setup picture (Figure 2.5).

The fluence used to irradiate the sample was $1 \mathrm{~J} / \mathrm{cm}^{2}$, which was controlled by means of a set of calibrated neutral density filters. Additionally, the cross-section of the pulsed beam used is slightly elliptical, with a nominal beam diameter of $15 \mathrm{~mm}$, and a beam propagation factor lower than two. As it was mentioned in Chapter 4, to reduce optical aberrations and increase the spatial uniformity, the beam passes through a $2 \mathrm{x}$ all-mirror beam expander. In addition, an iris of $6 \mathrm{~mm}$ diameter is placed before the focusing optics. To control the irradiation time, an electronically controlled shutter was utilized. After 15min of synthesis, in both protocol cases a brown-like coloured colloid was obtained.

Once the colloid was ready, we have directly continued with the post-processing stage. For this, we have used the colloid to fill a $10 \mathrm{ml}$ quartz cuvette with planar walls with a thickness of $1 \mathrm{~mm}$, which present a $90 \%$ transmittance in the spectral range from $200-2500 \mathrm{~nm}$. Therefore, the cuvette was irradiated using the light from the Ti:Sapphire laser previously focused with a $75 \mathrm{~mm}$ focal length lens as it was 
described in Section 3.1. To avoid a non-homogeneous irradiation of the material, the colloid was stirred with a magnet during the photo-ablation process.

The incoming fluence used to irradiate the sample was $1 \mathrm{~J} / \mathrm{cm}^{2}$ calculated under free propagation, but using the Barcikowski's theoretical approach for calculating the beam radius when a pulsed laser beam is focused into a liquid environment, without considering the interfaces air-quartz, quartz-liquid presented in this procedure, the approximate value reaches $0.6 \mathrm{~J} / \mathrm{cm}^{2}$ in both protocol cases.

\subsubsection{Preparation of nanofluids}

Five different nanofluid were prepared during the two protocols. Three of them synthesized by PLAL and the PPLI stage without and with the surfactant, and the remaining two using commercial NPs mixed with the commercial heat transfer fluid.Table 6.1 lists the investigated nanofluids.

Table 6.1: Samples under study.

\begin{tabular}{cc}
\hline Sample & Details \\
\hline nanofluid 1 & PLAL in Therminol VP-1 \\
nanofluid 2 & PLAL-PPLI in Therminol VP-1 \\
nanofluid 3 & commercial AuNPs in Therminol VP-1 \\
nanofluid 4 & PLAL-PPLI in Therminol VP-1+TOAB \\
nanofluid 5 & commercial AuNPs in Therminol VP-1+TOAB \\
\hline
\end{tabular}

The idea to create the five different nanofluids was to compare its characteristics. Since in the market is not possible to find Therminol VP-1 with gold NPs created in it, but is possible to find commercial gold NPs and mix them with the liquid. We have mixed in Therminol VP-1 and Therminol VP-1+TOAB respectively commercial gold NPs stabilized in $0.1 \mathrm{mM}$ of PBS solution with a primary particle diameter of $50 \mathrm{~nm}$, from Sigma Aldrich. The choice of this specific kind of particles was made on the base that we needed highly dispersed NPs similar to those obtained by the synthesis method proposed in this research work, to ensure no further agglomeration. To prepare the mixture, the water content was removed from the aqueous suspension by evaporation on a hot plate. Once the gold NPs were dry, they were dispersed in Therminol VP-1 and Therminol VP-1+TOAB for the nanofluid 3 and the nanofluid 5 respectively, with the help of an ultrasound probe resulting in a high concentration. Then the nanofluid was diluted with pure Therminol VP-1 or Therminol VP-1+TOAB depending on the case, to achieve the same optical density and solid content that the one obtained by using the synthesis approach proposed. 
The optical density was calculated by measuring the transmission of a laser beam centered at $635 \mathrm{~nm}$ (Thorlabs, Model CPS180) with a photo-diode (Thorlabs, Model $\mathrm{SM} 05 \mathrm{PD} 1 \mathrm{~B})$ in a rectangular quartz cuvette.

\subsection{Results and discussion}

The important characteristics to be studied in this work were related to the material's purity, the possibility to create a novel material with a higher thermal conductivity than the base fluid, and mitigate the agglomeration phenomena that could make this material suitable for real applications. To this end, it was important to analyze the morphology, mean size, size dispersion, crystalline structure, and stability through the time.

The techniques used to analyse the samples were: transmission electronic microscopy (TEM), energy dispersive x-ray spectroscopy (EDX), high resolution transmission electronic microscopy (HRTEM), selected area electronic diffraction (SAED) and dynamic light scattering (DLS).

In order to analyze in depth the issues discussed here, is firstly mandatory to recognize if we can find evidence of a chemical reaction between the NPs and the liquid environment during the production process, leading to the synthesis of a poor quality material and fabrication of by-products in the synthesis that eventually could change the nanofluid characteristics. To this end, we have used three different characterization techniques (EDX, HRTEM, SAED) to look for any typical evidence of chemical reaction such as the appearance of a lot of elements of the base liquid or crystalline defects. In this context, the preparation of the sample and vacuum the conditions in the TEM ensures that just solid content is under study. If any by-product is synthesised in an uncontrolled way during the production process, the created material should remain in the TEM's grill allowing the opportunity to be seen not just in the TEM's image, but also in an elemental analysis such as EDX. From a statistical point of view and considering that any product presented in the samples under study should represent a possible active core to generate further chemical reactions, the crystalline and elemental analysis is going to be performed only to the final products nanofluid 2, nanofluid 4 and nanofluid 5 .

In Figure 6.1, we can see the representative spectra of the 3 samples, nanofluid 2, 4 and 5. Where some elements aside of gold $(\mathrm{Au})$ are detected, among them there is carbon $(\mathrm{C})$, cooper $(\mathrm{Cu})$, iron $(\mathrm{Fe})$, chromium $(\mathrm{Cr})$, potassium $(\mathrm{K})$ and bromide $(\mathrm{Br})$. However, the presence of cooper and carbon can be due to the grid substrate, the 


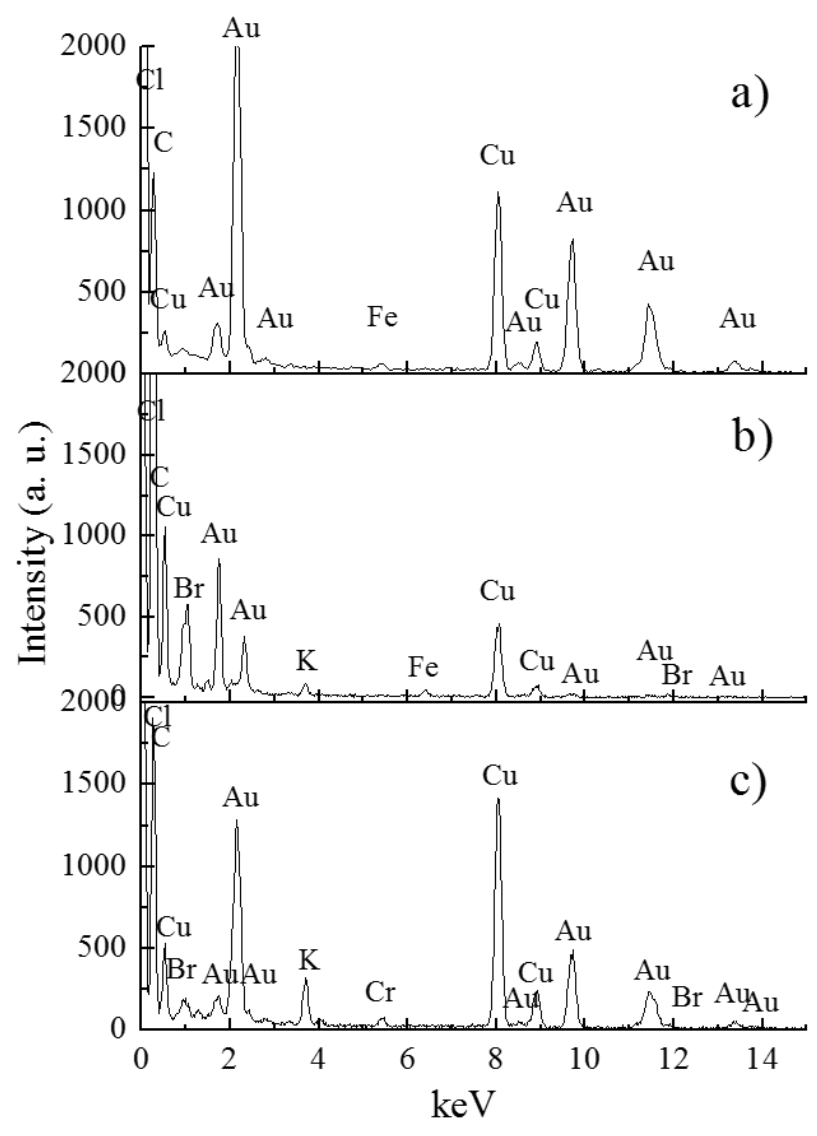

Figure 6.1: EDX spectra of a) nanofluid 2, b) nanofluid 4, and c) nanofluid 5 (already published in [1]).

TEM's grid used was made of cooper and contains a carbon film to immobilize the solid content on it. Iron and chromium can be detected because the microscope objective lenses are made of it. The presence of potassium and bromide in Figure 6.1(b, c) can be attributed to chemicals used to stabilize the nanofluids. For the nanofluid 2 , the first logical conclusion that can be extracted from its spectrum is that there is no any byproduct due to we are just observing gold and the elements forming the TEM's grid or the TEM's objective lenses. Nevertheless, is important to remember that Therminol VP-1 is an eutectic mixture of biphenyl and diphenyl oxide, and biphenyl is a molecule with a high content of carbons in its structure being composed of two benzene rings connected through a single bond as it is depicted in Figure 6.2. During PLAL fabrication, due to the high intensities used is possible the dissociation of this molecule resulting fabrication of carbon-based by-products. However, a 
detailed observation of TEM pictures demonstrate that after the production process, there is no evidence of amorphous structures aside of the particles, but the carbonbased TEM grids could be still hiding the presence of a low amount of carbon-based by-products. Therefore we can considerate that there is no evidence of creation of large amounts of carbon-based by-products,

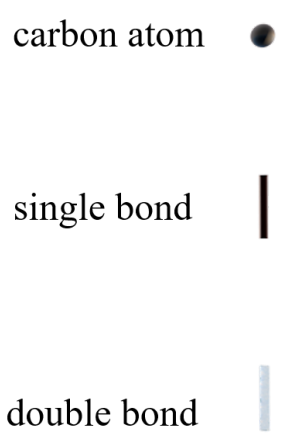

Figure 6.2: Biphenyl structural formula.

For the nanofluid 4, the presence of bromide can be attributed to the use of TOAB. Since it is a polymeric chain that surround the created NPs is possible that could remain as a solid specie in the TEM's grid and its presence is evidenced by the detection of one of its fundamental elements. For the nanofluid 5, as in the case of nanofluid 4 , the presence of bromide can be attributed to TOAB, but also is important to notice that as in nanofluid 4 the intensity for the $\mathrm{Au}$ peaks is lowered in comparison with the EDX spectrum of nanofluid 2, it could also represent a second evidence that the polymeric chain of TOAB is indeed surrounding the gold NPs, evidencing that the functionalization was successful.

In the other hand, the promotion of chemical reactions between the solid target and the base fluid can be evidenced through the promotion of defect formation in the solid crystals contained in the colloid. The thermal fluid and the surfactant are molecular systems that does not present a crystalline structure. Therefore, the only noticeable crystalline structure should belong to gold, its crystalline characteristics are the following: $\mathrm{Au}$ is its symbol which comes from the latín Aurum, it has 79 electrons in the electronic configuration $[X e] 4 f^{14} 5 d^{10} 6 s^{1}$ which means that naturally is not so reactive, it has an atomic radius of $0.174 \mathrm{~nm}$, and a face centered cubic crystalline structure or (FCC). According to the database of Joint Committee on Powder Diffraction Standards (JCPDS), the file for FCC Au crystals (JCPDS : $4-784$ ), revels that using $\lambda=15.4056 \mathrm{~nm}$, the inter-planar spacing and Miller index of its crystalline plane families is listed in Table 6.2 . 
Table 6.2: Miller index and inter-planar spacing ( $d$-spacing) recorded in (JCPDS:4784) for FCC Au crystals.

\begin{tabular}{lccc}
\hline$d-$ spacing $(\AA)$ & $h$ & $k$ & $l$ \\
\hline 2.35500 & 1 & 1 & 1 \\
2.03900 & 2 & 0 & 0 \\
1.44200 & 2 & 2 & 0 \\
1.23000 & 3 & 1 & 1 \\
1.17740 & 2 & 2 & 2 \\
1.01960 & 4 & 0 & 0 \\
0.93580 & 3 & 3 & 1 \\
0.91200 & 4 & 2 & 0 \\
0.83250 & 4 & 2 & 2 \\
\hline
\end{tabular}

As it was described in detail in Subsection 3.3.1., HRTEM allows the observation of distances under $1 \mathrm{~nm}$, the required resolution to observe a periodic arrangement of atoms in a crystal, and measure the distance between chains of atoms that are mathematically represented as crystalline plane families.

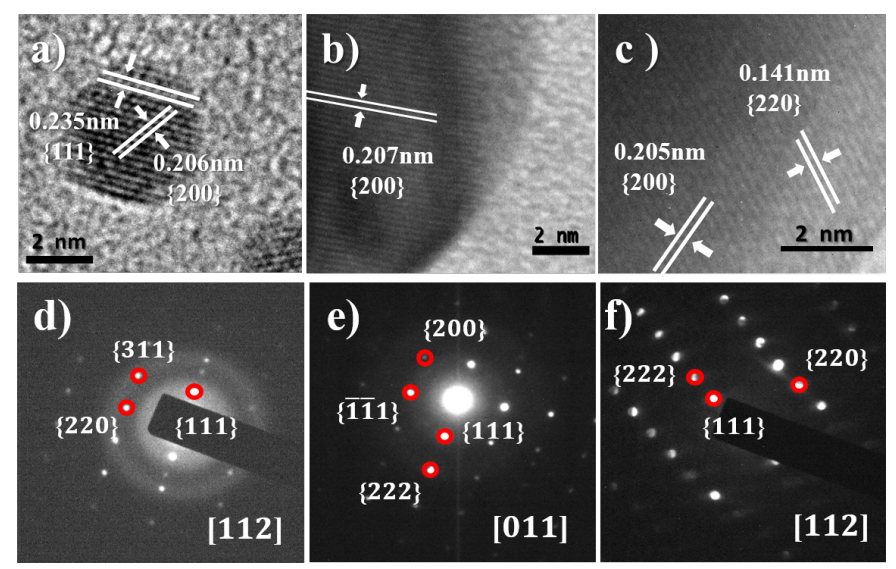

Figure 6.3: HRTEM micrographs of NPs (top) and SAED patterns taken of NPs (bottom). From left to right for nanofluid 2, 4 and 5. (already published in [1]).

In Figure 6.3, a set of representative HRTEM micrographs of the 3 samples, nanofluid 2, 4 and 5. Where some plane crystalline families can be identified.

For nanofluid 2 , the families (111) with $d-$ spacing $=0.235 \mathrm{~nm}$ and $(200)$ with $d-$ spacing $=0.204 \mathrm{~nm}$ can be appreciated. From an statistical analysis, no other crystalline families could be determined, or crystalline families which don't belong to the Au FCC crystalline structure. For nanofluid 4, obtaining images of HRTEM was more difficult to the case of the first sample, and just one crystalline family could 
be identified, (200) with $d-$ spacing $=0.204 n m$. For nanofluid 5 , as in the previous sample, HRTEM images were difficult to obtain but in this case two crystalline families were observed, (200) with $d-$ spacing $=0.204 \mathrm{~nm}$ and $(220)$ with with $d-$ spacing $=0.144 \mathrm{~nm}$. The results are very interesting due to, assuming that there has been a reaction between gold and carbon or bromide during the production process, at least the $d$-spacing should be modified showing the same periodic behavior for different crystalline planes, i. e. the all of the crystalline planes in gold would show the same $d-$ spacing plus a minimal distance $\Delta d$ constant between all of them or many punctual crystalline defects would be observed in the most of the studied particles. In the absence of this phenomenon, we could extrapolate that the synthesized particles of $\mathrm{Au}$ in Therminol VP-1 and Therminol VP-1+TOAB through the used approach seems to be free from important chemical reactions between the solid target and the liquid environment, that cause the creation of non-pure nanomaterials (it should be understood as pure nanomaterial, a nanostructure which present the same crystalline structure that the bulk). Another important but not evident result was the difficulty on the acquisition of HRTEM images in nanofluid 4 and 5, when an HRTEM image is difficult to obtain, this may be due to various phenomena, a big thickness of NP which could prevent the right visualization of crystalline planes, NPs position which prevents the clear visualization of any crystalline plane family, the obstruction of any amorphous body in the particle-electronic beam interaction path or technical phenomena belonging to the microscope like not the right vacuum value, not a right adjustment of the TEM's grid, etc. In summary, if we consider that all of the technical features of the microscope were optimized, that we have found the right position and considering that the zone where the analysis is performed doesn't show a strong black colour which would evidence a big thickness. The last possible option is that actually the polymers surrounding the particle, which are invisible to HRTEM would be obstructing the interaction between the electronic beam and the sample. Considering that HRTEM images would provide useful information but maybe not completely accurate due to the presence of surfactants, SAED analysis was performed to ratify the conclusions extracted from HRTEM and EDX analysis.

In Figure 6.3(bottom), is depicted a set of crystallographic spectra of nanofluid 2, 4, and 5. For nanofluid 2, the crystallographic families (111), (220), (311) from the FCC crystallographic geometry belonging to the gold crystals could be identified, observing the sample from the crystallographic plane (112). For nanofluid 4, the distance between first neighbors was not so clear and it was mandatory to measure 
distances between primary and secondary neighbours, and the crystallographic families found were (111), (200), (222) and (1111), observing the sample from the plane (011). For the nanofluid 5, the spectrum was so difficult to get, and at the first look the distance between primary and secondary neighbours does not look to match with the distances for FCC gold when the sample is observed from the crystallographic plane (112), nevertheless, considering that the most of the diffraction points in the pattern seem to be elliptical instead of completely circular, it was clear that two possible things would be happening, one of them and the most probable one is that maybe the sample was not perfectly placed for make the SAED analysis, or the presence of any chemical element would affect the crystallographic structure of the sample. However, the distance between all of the diffraction points was not equidistant making more possible the first option. Based on this assumption, we have determined the observation of the following crystallographic families (111), (222), (220), that should appear under normal conditions. The results extracted from SAED analysis ratify the hypothesis formulated from the extraction of HRTEM and EDX results. Based on the detailed observation of each result we can summarize that there is not an important fabrication of by-products or promotion of chemical reactions during the proposed synthesis route that eventually could alter the material's characteristics, and based on the functionalization or covering of gold NPs with TOAB was successful.

As a second characterization step and thinking about the stability of the material over time, the following analysis lies on the morphological characteristics of NPs immersed in the liquid environment and how it could affect the liquid dynamics through the time. The morphological studies were performed by analyzing TEM micrographs of both materials, the commercial and the manufactured material. At this point is also important to notice that during the post-processing stage in the synthesis proposed method, the morphological characteristics are modified and this change can result in an improvement over the material stability. Therefore, to determine the real influence of the post-processing stage in the synthesis route, the morphological, dynamic and thermal behavior analysis of the nanofluid overall resulting from PLAL, from the entire process and from the use of commercial particles using and not using TOAB is performed and compared through the time, to understand if it makes sense to use this synthesis approach beyond the theory.

TEM micrographs comparison depicted in Figure 6.4 show that there is a change between primary NPs morphology, apparently the NPs created by PLAL, and the NPs created by PLAL and further fragmented by the laser radiation present a morphology more spherical than commercial NPs, which makes perfectly sense due to 

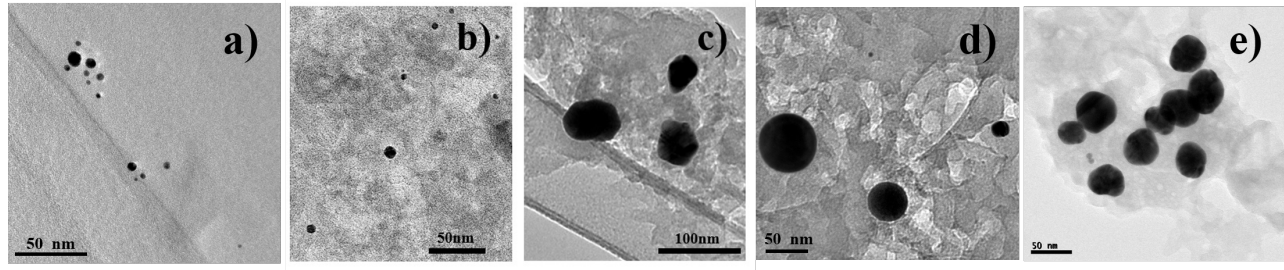

Figure 6.4: Comparison of TEM micrograph for a) nanofluid 1, b) nanofluid 2, c) nanofluid 3 (already published in [2]), d) nanofluid 4, e) nanofluid 5 (already published in $[1])$.

the phenomenons involved in NPs creation. While in chemical methods, the particles growth from the nucleation of gold atoms until reaching a desired size. In the processes used in this research work, the final stage of the particle formation consists in a release of energy to the liquid environment from an energetic structure, experiencing surface tension when finally the structure reaches and stable stage, leading to the spherical NPs shape. In the other hand, according to the compositional and crystallographic analysis, the nanofluid 1 is the only one which seems to be free from any extra amorphous structure surrounding the NPs. However, in nanofluid 2 the particles are surrounded by an homogeneous amorphous matrix while in nanofluid 3 and 5 the NPs seem to be surrounded by an amorphous structure which is just located where the nanostructure is. Nanofluid 4 in the other hand present a different behavior, apparently, the NPs are surrounded by an amorphous structure which is a mixture between the one presented in nanofluid 2 and the one presented in nanofluid 3 and 5.

The observation of the homogeneous amorphous matrix in nanofluid 2 and 4 could be an evidence of by-products formation during the post-processing stage used either during the first and the second protocol. The observation of such structure makes sense, since in the post-fragmentation process the laser beam is focused in the colloid, is possible that not just NPs suffer photo-fragmentation, also the molecules forming Therminol VP- 1 could be dissociated by the influence of the laser radiation. Apparently this phenomenon is not appreciated in the stage where PLAL is used because the most of the energy could be employed to detach material from the solid target instead of dissociating the fluid's molecules, since the laser beam is focused in the surface of the solid target and this in turn, absorbs the most of the delivered energy.

The observation of non-homogeneous amorphous structures surrounding the NPs in nanofluid 3 and 5 could be due to the visualization of the surfactant PBS used to stabilize the commercial nanostructures by surrounding them. Nevertheless, the 
amorphous structure surrounding NPs in the nanofluid 5 is more similar to the extra amorphous structure which is non-homogeneous that surrounds the NPs in nanofluid 3. This structure could be due to the visualization of an extra element, in this case TOAB. This surfactant, as it was mentioned before, is a ionic one and as the most of this chemical species, is conformed by long chains of carbons. In TEM, the carbon is an element which is not so visible unless it is presented in large amounts. If we consider that the surfactant envelope the NPs then is understandable that this amorphous structure surrounds the NPs in a non homogeneous way.

Moreover, is important to notice the separation between NPs in each sample. Apparently in nanofluid 5 the NPs are closer than in any other sample, this may be a consequence of the mixture of surfactants TOAB and PBS.

Additionally, the average size of NPs in each case seems to be completely different. Synthesized NPs look smaller than commercial NPS. To ensure a right size and size distribution of particles, DLS measurements were performed in the samples and compared. At this point is important to notice that since in equation 3.13, the diffusion coefficient for each sample is strongly related to the relation between the dielectric constant of the particle and the liquid environment, just as in the case of LDV measurement. Then, considering that the amount of TOAB dissolved in Thermonol VP-1 would not change its dielectric constant, it can be assumed that TOAB would be invisible to the DLS technique.

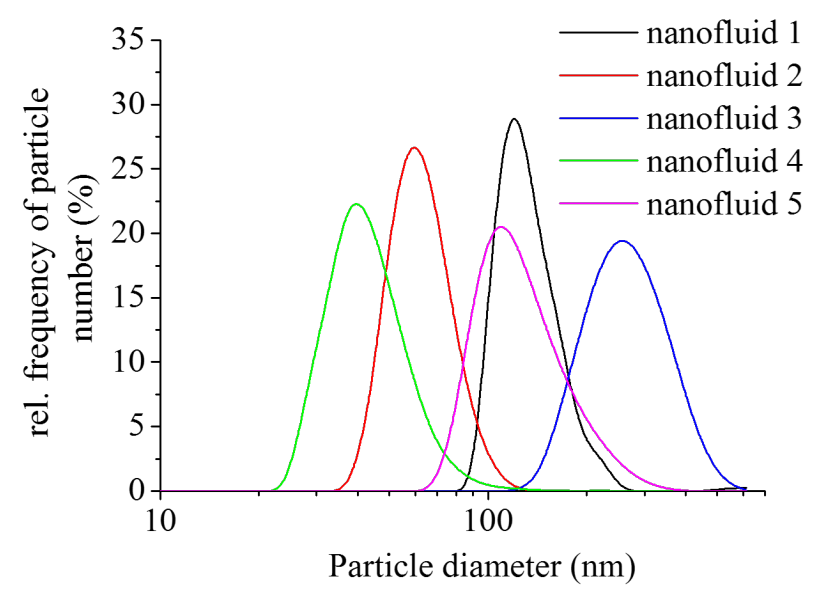

Figure 6.5: Comparison of size and size distribution of samples (already published in [2] and [1]).

The primarily sizes depicted in Figure 6.5, doesn't match with the sizes depicted in Figure 6.4 except for nanofluid 4. The reason for this, is the rapid agglomeration of 
particles in the rest of the samples. From the analysis of statistical size, the mean size and size dispersion of the formed clusters, nanofluid 4 present the smallest primary cluster size, $39 \mathrm{~nm}$ at the full width half maximum (FWHM) of the size distribution while the size dispersion was the shortest one with a value of $42 \mathrm{~nm}$. In contrast, the nanofluid 3 shows the biggest primary mean size of $108 \mathrm{~nm}$ and the highest size dispersion (about 124nm of FWHM). The addition of a surfactant allows obtaining smaller clusters of particles either in commercial nanofluids and nanofluids produced by the proposed approach.

The comparison between both experimental results leads to the natural conclusion that agglomeration is presented since the particles are created, at least in nanofluid 1,2,3 and 5, but showing a mitigation of agglomeration when TOAB is used for commercial nanofluids. The samples for the TEM images were prepared just after the production of the nanofluids, while the preparation and further measurement of DLS took at least 30min. During this time the particles started to form clusters, and Zetasizer nano ZS interpret the formation of clusters as a particle size growth. Due to the light scattering interpretation of the device explained in detail in Subsection 3.3.2, is understandable that a cumulus of particles will move slower than a single particle resulting in an interpretation of a single bigger particle. If DLS measurements are taken through the time, the dynamics of the agglomeration of particles can be determined.

In this context, one of the main goals of this research work was to try to find the necessary conditions to fabricate a nanofluid that could remain for prolonged periods of time without significant loss of stability, since this is a fundamental characteristic sought in fluids that can be used in real applications. Nevertheless, as it was mentioned above, for colloids composed with NPs eluding this phenomena gets more difficult, mainly due to the ratio particle surface to volume is so high that all the interactions are controlled by short-range forces. As a consequence of the attractive nature of the forces presented, when primary particles are suspended in a fluid they have a tendency to agglomerate when they come into contact due to their Brownian motion.

When the cumulus of particles get big enough, Stokes sedimentation is promoted over Brownian motion and the clusters tend to settle leading to fluid dynamics problems $[218,219]$.

The Figure 6.6 which represents a comparison of mean sizes of particles among the samples through the time, revels that particles which have been produced by the whole synthesis approach using TOAB as stabilizer, shows the lowest agglomeration 


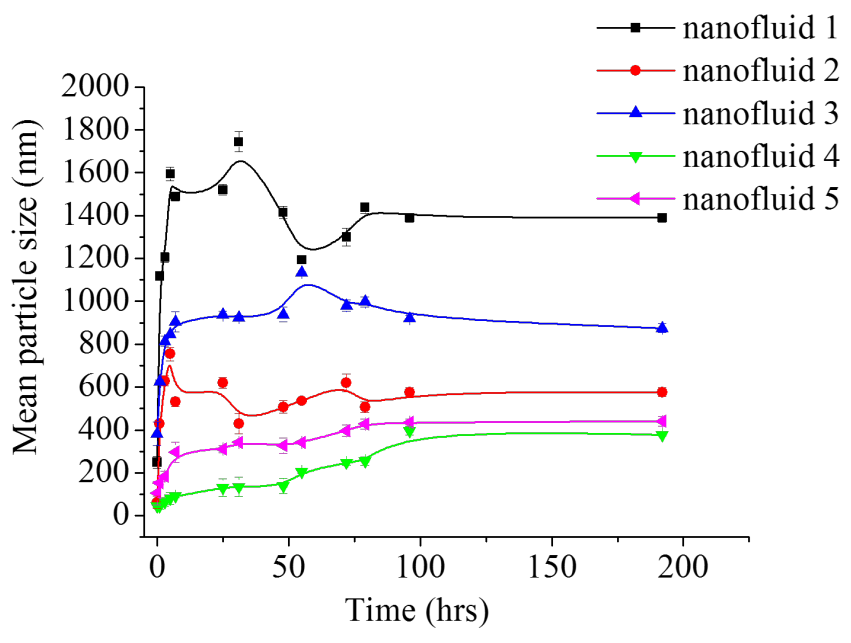

Figure 6.6: Comparison of mean particle sizes through the time (already published in [2] and [1]).

rate reported in the literature up to know, and therefore the highest colloidal stability, understanding as stability not just the thermodynamic sense of it but also in a strictly colloidal sense. Its first cluster's size recorded by DLS was $39 \mathrm{~nm}$ with a size dispersion of $42 \mathrm{~nm}$, and a final mean size of $370 \mathrm{~nm}$ where seems to reach an stability and no further agglomeration at $96 \mathrm{hrs}$.

For all of the samples, the biggest agglomeration behavior happens at the first $6 h r s$, once that time has passed, the agglomeration seems to stop and keeps constant for at least another $90 \mathrm{hrs}$. From a technological point of view, 90hrs is enough time to consider that a heat transfer fluid can be stable, since the fluid remains stationary just for periods of time of $24 \mathrm{hr} s$ under working conditions. Nonetheless, if we consider that the fluid should be stored for longer periods of time until this finally is used, then an extra experiment is required. Supposing that 1 month seems to be a fair store time, we have measured the cluster's size of nanofluid 4 after one month and it was approximately the same. Based on DLS analysis we can conclude that the addition of TOAB using the proposed synthesis approach largely mitigate the unwanted agglomeration phenomena, compared to NPs fabricated in the pure thermal oil without any additive, and the commercial nanofluid. In addition, nanofluid 4 cluster's size is considerably smaller (factor of 4) than those reported in previous works [208] using the same NPs material, base fluid and chemical additive, but different production method.

In the other hand, even when not all of the samples reach its stability at the same cluster size, being the sample just produced by PLAL the one which have 
reached the biggest particle cumulus size at $1500 \mathrm{~nm}$. None of them experience a sedimentation phenomenon, sedimentation phenomenon would be noticeable if bigger particles started to settle giving the opportunity to measure just particles with smaller sizes. In this sense, all of the samples showed a relatively good stability. Nonetheless, the agglomeration phenomenon doesn't just lead to stability problems, also leads to fluid dynamics issues and in this research work, could also represent a problem regarding to thermal conductivity enhancement.

As it was posed from the first time, one of the biggest goals of the here exposed research activity, is to propose a synthesis route to fabricate a novel nanofluid which present remarkable thermal features and a convenient stability to be used in real applications. Therefore, despite all the samples are stable it was mandatory to perform a measurement of their thermal conductivity. It was performed through the hot wire transient method (explained in detail in Subsection 3.3.3) using a KD2 Pro conductimeter (Decagon Devices Inc.), performing six measurements of each sample at $80^{\circ}$ to ensure an experimental error that lead us to a confidence level of $95 \%$. The measurements are represented in the Table 6.3.

Table 6.3: Thermal conductivity measured by hot wire transient method at $80^{\circ} \mathrm{C}$ (Table published in [2] and [1]).

\begin{tabular}{ccc}
\hline Sample & $k(W / m K)$ & $\Delta k(\%)$ \\
\hline Therminol VP-1 & $0.1280 \pm 0.0030$ & - \\
Therminol VP-1+TOAB & $0.1268 \pm 0.0030$ & - \\
nanofluid 1 & $0.1282 \pm 0.0015$ & 0.13 \\
nanofluid 2 & $0.1332 \pm 0.0014$ & 4.06 \\
nanofluid 3 & $0.1320 \pm 0.0018$ & 3.13 \\
nanofluid 4 & $0.1313 \pm 0.0011$ & 3.55 \\
nanofluid 5 & $0.1288 \pm 0.0018$ & 1.58 \\
\hline
\end{tabular}

From the results recorded by the method, is important to notice that as it was expected the thermal conductivity increases when NPs are suspended in the base fluid. Nevertheless, considering the original Maxwell's idea of the improvement of thermal conductivity through the transfer of kinetic energy promoted by the contact of two systems with different thermal conductivity values. It makes perfect sense that the sample synthesizes by the proposed fabrication method presents the highest thermal conductivity, due to it reaches the smallest agglomeration size through the time, and therefore it presents the highest surface volume ratio interacting with the liquid environment. Nonetheless, is also important to notice that the addition of TOAB result in the reduction of Thermal conductivity improvement, mainly due 
to the capping action of the surfactant over the metal NPs don't allow them to interact directly with the base fluid. Therefore, the use of TOAB helps to mitigate the agglomeration phenomenon between the particles but in the other hand also hinders the possible thermal interaction between metal NPs and the base fluid. The immediate conclusion to this results is that the amount of TOAB should be lowered for further experiments, nevertheless the amount of TOAB was optimized to get the best stability and thermal enhancement performance. Therefore, a more realistic conclusion is that the use of ionic surfactants to avoid the agglomeration is required and as it was demonstrated in the experiments performed, its use is highly compatible with the proposed synthesis approach. But the particular use of TOAB which presents carbonic chains of at least 32 atoms may not be the best option to achieve the best thermal conductivity enhancement. Therefore is necessary the use of another ionic surfactant which can support working temperatures similar to those reached in SPCs as TOAB but with smaller polymeric chains.

\subsection{Conclusions}

The most important contributions of this research work lies on the creation of two novel materials, one of them presents a thermal conductivity enhancement of $4.06 \%$ over a commercial heat transfer fluid used in SPC, and a considerably stability enhancement, and the second one presents a thermal conductivity enhancement of $3.6 \%$ over the available commercial heat transfer fluids, and the best stability of nanofluids composed of gold NPs and an eutectic mixture of biphenyl and diphenyl oxide. The successful synthesis of this nanofluids by PLAL and post-fragmentation stage, without using any chemical additive and functionalizing them with a ionic surfactant. The synthesis proposed in the research work do not promote the formation of large amount of by-products or chemical reactions between the liquid environment and the solid target that could represent problem due to further modification of the nanofluid. Moreover, the sizes of particle's clusters reached for the sample produced by the proposed synthesis approach are not easily achievable by commercial nanofluids due to a rapid agglomeration phenomenon.

Additionally, the difference in thermal conductivity values obtained for different particle's clusters sizes, ratifies the Maxwell's theory of thermal conductivity enhancement and Gao et. al. [184] observations on the enhancement properties in nanofluids depending on size and shape of the particles and clusters of particles in the nanofluid. In the other hand, the decrease in thermal conductivity when TOAB is used points 
out the necessity to keep looking for the best ionic surfactant which promotes steric repulsion but also allows the enhancement of thermal conductivity of the base fluid. Finally, the results presented in the research work suggest that the employed synthesis method represents an advantage over conventional methods to produce NPs in heat transfer fluids that may potentially have a significant impact on the improvement of harvesting efficiency of solar light with nanofluids. 


\section{Chapter 7}

\section{Direct laser synthesis of gold nanoparticles and immobilization on graphene sheets}

This chapter provides a review of the work related to the immobilization of ligand-free metal NPs in graphene-oxide by PLAL, performed during the PhD studies.

The idea of this work was to manufacture nanometric assemblies of carbonaceous materials and metal NPs due to its remarkable characteristics. It is well known that materials based on carbon with periodic structures exhibit a strong electrical conductivity response and as a result are widely exploited in the electronic industry. Among them, the most interesting carbon-based structure is graphene, since this material is highly conductive and its weight is practically negligible due to its mono-atomic thickness and it could be as large in two dimensions as desired. In the other hand, metal nanostructures present besides of their magnetic, electrical and thermal characteristics, a remarkable optical response. At nanometric sizes, the most of metal structures present optical properties that differ from the bulk. As it was described in Chapter 3, when the metal NPs undergo the presence of an oscillating electromagnetic field, the external electrons forming the metal bonds follows the oscillation perpendicular to the metal lattice. This process is maximized at frequencies of the light that match with the particle's size, the process is known as surface plasmon resonance. After absorption process, the plasmonic effect is diminished resulting in light scattering or heating of the particle. The morphology of the particle could also promote another effects such as multiple plasmonic resonances and strong absorption of a wide range of wavelengths. And minimal sizes can promote the increase of quantum confinement in the metal structures resulting in emission of photons with high energies after the absorption of relatively long wavelengths. Due to these remarkable optical proper- 
ties, metal nanostructures are subject of intense research, with unique applications in material sciences, biological imaging, and electronics.

The scope of this research work lies on the possibility to create a primitive nanocomposite of graphene and metal NPs. Considering that the traditional production of graphene-metal primitive assemblies is achieved by solution mixing of the nonenvironmental friendly reduction of metal salts and carbonaceous suspensions, resulting in the anchor of metal NPs in carbonaceous species [220]. The main goal is to synthesize metal NPs promoting a direct immobilization onto graphene sheets in a single reaction stage using PLAL. Since the conventional methods present precursor residues in the NPs growth and tend to reduce its surface activity and promote cross-chemical undesired effects, PLAL promise to be a possible alternative to avoid the NPs surface activity reduction due to NPs can be produced without the use of any external chemical specie, moreover when metal NPs are created in water, the resulting structures present a ligand-free surface, leading to obtain nanomaterials with higher possibilities to present either remarkable electronic or optical characteristics. In this research work, PLAL is used to create gold ligand-free NPs that are directly anchored to graphene sheets. The synthesis is performed with a laser mean power of $40 \mathrm{~mW}$ on a gold disc of thickness $1 \mathrm{~mm}$ and diameter $6.5 \mathrm{~mm}$ (99.99\% purity) surrounded by a mixture of deionized water $8 M \Omega$ and graphene oxide (GO) prepared from graphite powder (natural, universal grade, 200 mesh, 99.9995\%) by the Hummers method [221]. The NPs produced were naturally hooked and immobilized on the graphene oxide surface, the presence of graphene oxide during the synthesis method seems to influence the NPs size allowing to obtain particles with an average size of $2 \pm 0.5 \mathrm{~nm}$. Moreover, the interaction between the laser radiation and the graphene oxide promotes its partial reduction by taking out oxide functional groups in the GO but keeping the wide inter-layer spacing that characterize the electronic decoupling of individual layers in this material, which in some cases leads to superconductivity effects. In summary, this research work constitutes a green alternative synthesis and an improvement over conventional synthesis approaches of graphene-metal assemblies and a practical methodology which may inspire future developments of efficient decoration of graphene and related materials with metal nanostructures.

\subsection{Introduction}

Since the discovery of carbonic periodic organized structures, carbonaceous materials had been widely exploited in different fields, from their use for drawing to their use for 
catalysis. But it was not until Andre Geim and Konstantin Novoselov discover in 2004 the easiest way to get an stable material with a mono-atomic thickness composed just by carbon atoms (graphene), that the electronic world changed drastically leading to the fabrication of record breaker devices. With the arrival of a material with outstanding electronic properties, it did not take long for scientists worldwide to try to exploit it's unique properties in several fields. But graphene is not a material that does not allow further modifications. In contravention, graphene can be functionalized, or even its structure can be modified to achieve different characteristic.

Among the most interesting modifications, the decoration of graphene with ligandfree metal nanoparticles (MNPs) is envisaged as a technological revolution, due to the hybridization of the unique graphene conductivity and the high chemical and physical reactivity of ligand-free MNPs. The principal features that can be exploited nowadays are the spatial 3 dimensional localisation control over the surface plasmon resonance of the MNPs [222], the enhancement of light absorption from graphene sheets [223], the bio-conjugation with many molecules to produce bio-sensors based on the plasmonic effect of MNPs [224-228], and the improvement of the catalytic activity of graphene due to the ligand-free surface of MNPs [229, 230].

Traditionally, the way to decorate graphene sheets with MNPs is based on a solution mixing protocol that follows two stages, the fabrication of MNPs and graphene separately and further mix. This method neither ensure the correct immobilization of MNPs onto graphene surface nor the presence of pollution and by-products created in the MNPs and graphene synthesis. Additionally, the manufacture of graphene extremely pure in large quantities, still requires large economical investments. To face this drawbacks, in-situ synthesis methods based on the reduction of metal salts and highly oxidized graphene derivatives had been proposed [231-233]. But these methodologies does not avoid the presence of co-ligands or surfactants in the nanoparticles formed, used to enhance the stability and avoid aggregation [234,235], leading to poor reactivity enhancements, the diminish of optical features of MNPs, or even the promotion of crossed-chemical effects due to MNPs are normally covered with surface absorbed ligands [230]. All of this without considering the environmental implication of producing by-products and chemical waste during the synthesis of the material $[110,236]$. To face this issues, in this research work we propose an alternative approach based on PLAL to synthesize ligand-free MNPs that directly get anchored on graphene's surface [237], and allows the presence of almost any kind of colloid during the manufacture [238-241], even when the colloid is composed by water and graphene derivatives. 
Under normal conditions, in PLAL a pulsed laser beam interacts with both the liquid environment and a solid target where the NPs are going to be created from. Nonetheless, the most of the energy is driven to the surface of the solid target, when the radiation hits the surface's target, the interaction between the strong electromagnetic field of the pulsed radiation and the atoms at the material's surface, promotes the detachment of material from the solid target's surface in the form of NPs through Coulombian explosion, melting and further evaporation or mechanical secondary effects. When metal NPs are surrounded by water, the most of them show a lack of electrons in the surface [190,242]. Considering that ligand-free gold NPs present an increment of the specific surface activity due to the lack of ligands on the surface, the particles can be retained in ordered matrix just like the surface of graphene sheets by the combination of different effects. The electrostatic interaction between the NPs and the graphene that leads to strong immobilization, the $\pi$-electron aromatic system found in graphene and derivatives interacts with the d-orbitals of the MNPs [243], or the allocation of NPs at the defects found in graphene [244].

The decoration of Au ligand-free NPs created separately by PLAL and subsequently mixed with graphene derivatives has been studied [245]. However, in a PLAL experiment in which the liquid environment is composed by graphene-oxide sheets dispersed in water, the water can promote the formation of ligand-free MNPs, but the laser will also interact with the graphene-oxide sheets. Since the graphene derivative is oxidized graphite in which oxygen molecules are inserted between layers of periodically ordered carbon atoms, and further separated by few superimposed layers when is reduced. The most probable interaction process is the detachment of oxygen functional groups out in the graphene-oxide sheets. Leading to a material which in some cases could present superconductivity effects [246,247]. Therefore, the use of PLAL to create ligand-free MNPs in a solution composed by water and graphene-oxide could lead to the synthesis of a nano-composite of ligand-free MNPs immobilized in reduced graphene-oxide.

In this work, we focused on producing gold ligand-free NPs anchored to partially reduced graphene-oxide sheets in a single reaction step. The impact of femtosecond radiation-based technique controlling the production of ligand-free Au NPs in an ambiance filled with graphene-oxide sheets is studied. For this purpose Au-NPs were produced in two different liquid environments for its further comparison, deionized water and a graphene-oxide solution. Our results demonstrate that is possible to create ligand-free MNPs directly anchored in partially reduced graphene-oxide sheets in a single reaction process. The methodology used provides an effective way to obtain 
graphene-metal assemblies that potentially can be used in several applications where crossed-chemical effects is a problem that needs to be avoided.

\subsection{Experimental setup}

\subsubsection{Material synthesis}

All of the experiments performed during this research work followed the same protocol, which follows the classical procedure to perform PLAL. In all of the cases, a gold disc of thickness $1 \mathrm{~mm}$ and diameter $6.5 \mathrm{~mm}$ (99.99\%purity) was used as a solid target to make ablation in it, the target was surrounded by the liquid medium (either $\mathrm{H}_{2} \mathrm{O}$ or $\mathrm{H}_{2} \mathrm{O}+$ graphene-oxide) and the ejected material that comes from the ablation process was captured in the surrounding medium.

The gold disc was placed at the bottom of a glass chamber filed either with deionized $\mathrm{H}_{2} \mathrm{O}, 8 \mathrm{M} \Omega$ or $\mathrm{H}_{2} \mathrm{O}+$ graphene-oxide, depending on the experiment. The thickness of the fluid layer above the gold target in all of the cases was set to be $2 \mathrm{~mm}$. Then the target was irradiated from the air-liquid interface as it is depicted in Figure 2.5(right side) with the femtosecond laser beam focused with a $35 \mathrm{~mm}$ lens onto the target surface plane while the sample is moving at a scanning constant velocity of $0.45 \mathrm{~mm} / \mathrm{s}$ by means of a $2 \mathrm{D}$ motion controlled stage. Unlike cases where nanostructures were generated in transparent liquids, the colloidal mixture $\mathrm{H}_{2} \mathrm{O}+$ graphene-oxide has an influence on the laser propagation, acting as a neutral optical density filter resulting in a decrement of the amount of power delivered to the surface. Therefore, a shorter focus length is needed to promote detachment of the target's surface. The optimized incoming mean power of the laser beam was set to be $40 \mathrm{~mW}$. Since the result of the obstruction of light's path by the graphene-oxide sheets is just considered to be based on absorption of energy and not in scattering or further linear or non-linear optical effects, is possible to use the Barcikowski's approach to get the value of the energy delivered to the solid target, replacing the presence of graphene-oxide sheets by a neutral optical density filter, which promotes the same energy decrement that $2 \mathrm{~mm}$ of thickness of the carbonic solution, reaching an incoming mean power value of $10 \mathrm{~mW}$. The assumption of just considering linear interactions where absorption is the strongest, is based on a simple reason. Since the laser beam doesn't promote super-continuum in the liquid layer above the solid target which might be an evidence of non-linear optical effects, is possible to ensure that the focusing conditions don't allow the concentration of energy required to induce important non-linear optical effects that eventually could consume energy. 
In the other hand, to ensure the strongest absorption of energy by the solid target instead of any other element in the liquid ambiance or to avoid dispersion induced by the liquid itself or optical elements used to drive the laser beam to the sample, we have compensated the dispersion by changing the relative position of two fused silica Brewster prisms located in a post-compression stage in the laser. Macroscopically, the optimal position of Brewster prisms results in the maximum detachment of material from the solid target.

\subsubsection{Preparation of samples}

To understand the influence of producing ligand-free MNPs under the presence of graphene-oxide, four different experiments were performed. The main difference in each experiments is the energy used to create the nanomaterials and the liquid environment in which the material is created.

In the first experiment, a mixture of deionized water $8 M \Omega$ and graphene-oxide was used as a liquid environment. The graphene-oxide was prepared from graphite powder (natural, universal grade, 200 mesh, 99.9995\%) by the Hummers method [221]. The resulting in a colloid formed by $\mathrm{H}_{2} \mathrm{O}$ and homogeneously dispersed graphene-oxide sheets $(1 \mathrm{mg} / \mathrm{ml})$. The measured value of mean power used to induce ablation in the solid target before crossing the interface liquid-air was $40 \mathrm{~mW}$.

In the second experiment, deionized water $8 M \Omega$ was used as the liquid environment, and the measured mean power before the liquid-air interface was set to be $40 \mathrm{~mW}$. The morphological properties of the particles were compared with the MNPs obtained in the first experiment.

In the third experiment, deionized water $8 M \Omega$ was used as the liquid environment, and the measured mean power before the liquid-air interface was set to be $10 \mathrm{~mW}$, to explore if a lowering in the mean power promoted by the presence of graphene-oxide sheets in the liquid environment could also promote a change in the MNPs.

In the fourth experiment, MNPs were prepared using deionized water $8 M \Omega$ as liquid environment and therefore were mixed with a colloid composed of water and graphene-oxide similar to the one used in the first experiment to confirm if the immobilization of particles is related to the synthesis process.

\subsection{Results and discussion}

The characteristics of synthesized materials to be studied during this research work were related to the possibility to create ligand-free MNPs that are directly immobi- 
lized in reduced graphene-oxide sheets in a single reaction stage. The utilization of graphene-oxide sheets dispersed in water as a liquid medium, imply possible modification in the MNPs production process. Therefore to understand which is the influence of graphene-oxide in the PLAL process is mandatory to perform crystalline, compositional and morphological analysis of the produced material, and compare the obtained results with results obtained using the traditional PLAL procedure to synthesize MNPs without the presence of this graphene derivative.

The techniques used to analyse the samples were: transmission electronic microscopy (TEM), scanning electronic microscopy (SEM), high resolution transmission electronic microscopy (HRTEM), high-angle annular dark-field scanning transmission electronic microscopy (HAADF-STEM), fast mapping with silicon drift detectors (FS Mapping), X-ray powder diffraction (XRD), energy dispersive x-ray spectroscopy (EDX) and X-ray Photoelectron Spectroscopy (XPS). The samples used for TEM related analysis were deposited in a carbon-coated copper-based TEM grid. And the samples used for XRD were dried by evaporating the water content. The samples used for SEM were deposited in an aluminium based SEM sample holder.

From a morphological point of view, the addition of any external molecule to the base fluid can promote its adsorption by the free ligands located at the surface of MNPs during the particle's growth process that takes place in PLAL. The adsorption of those molecules can be used to functionalize the MNPs, control their shape, their size or even to control their chemical reactivity.

The micrograph displayed in Figure 7.1 shows the size and shape of MNPs created by PLAL under the presence of graphene-oxide. A statistical analysis shows that in general, the shape of MNPs is spherical, the average size of the particles is $2 \pm 0.5 \mathrm{~nm}$ and in some special cases the maximum particle size found was $24 \mathrm{~nm}$. The particles are homogeneously distributed all along the graphene-oxide sheets and there is no evidence of particles out of that place.
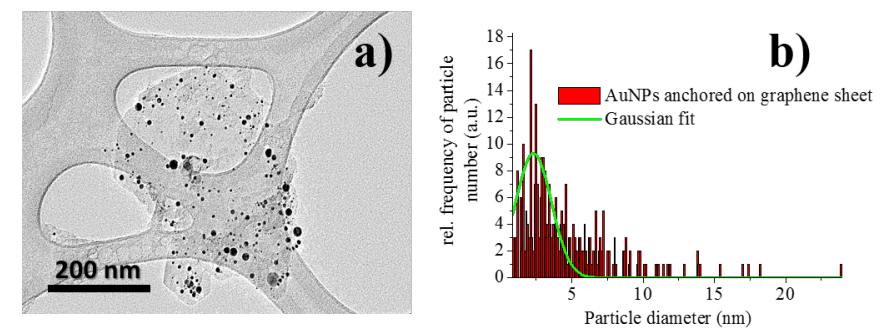

Figure 7.1: a) TEM micrograph of Au-NPs hooked on graphene sheets, and b) histogram of the NPs size distribution. 
In the other hand, the Figure 7.2 shows the TEM micrograph of MNPs created by the PLAL method under classical conditions without the presence of graphene-oxide, and a mean power of $40 \mathrm{~mW}$. The shape of the particles is similar to those obtained under the presence of graphene-oxide, but the sizes achieved are completely different. In this case, the average size was $26 \mathrm{~nm}$ with a size distribution of $46 \mathrm{~nm}$.
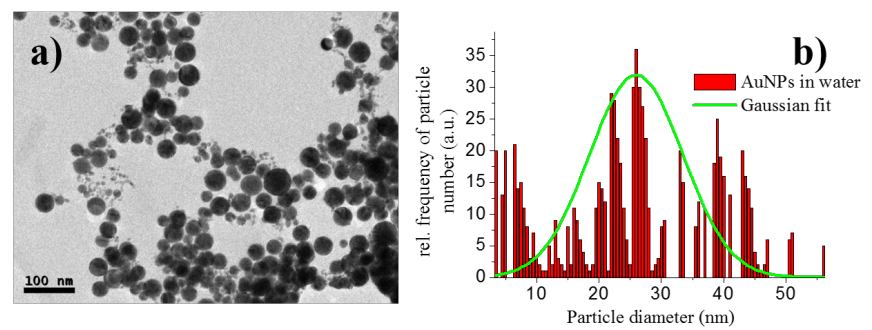

Figure 7.2: a) TEM micrograph of Au-NPs produced using $\mathrm{H}_{2} \mathrm{O}$ as liquid environment and a mean power of $40 \mathrm{~mW}, \mathrm{~b}$ ) histogram of the NPs size distribution.

In the third experiment, where the influence of the lowering in the power used over the particle's shape and size is studied, the micrograph depicted in Figure 7.3, reveals a lowering in the created particle's size achieving an average size of $6 \mathrm{~nm}$ with a size distribution of $2 \mathrm{~nm}$, and the same shape found in the previous results.

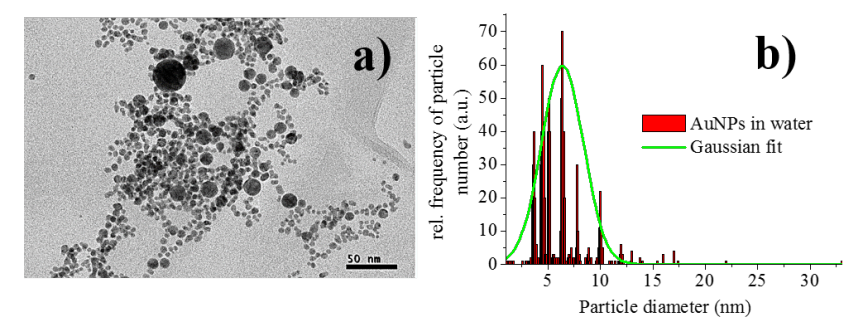

Figure 7.3: a) TEM micrograph of Au-NPs produced using $\mathrm{H}_{2} \mathrm{O}$ as liquid environment and a mean power of $10 \mathrm{~mW}, \mathrm{~b}$ ) histogram of the NPs size distribution.

The spherical shape of the particles synthesized by laser ablation in both liquid environments is due to, during the synthesis the fragmented material is released from a bulk material with an excess of energy and suspended in a liquid ambiance which has a certain density, undergoing a surface tension effect leading to a final shape similar to spheres.

Under the presence of graphene-oxide sheets, the shape doesn't seem to change or be modified. Since the graphene-oxide sheets are strong periodically organized structures with large 2 dimensional sizes (in the order of micro meters), the possible 
coating of the particles surface due to the interaction between free ligands in the particle and carbonic structure doesn't seem to have the potential to occur all over the particle's surface, there is no reason for the particles to don't release their excess of energy to the water instead of the graphene-oxide sheets and therefore, experience the same surface tension that in the case where there is no presence of graphene-oxide.

In the other hand, the coating of the particles surface seems to be possible but through the interaction of a limited number of free ligands in the particle's surface and the carbonic structure, this may lead to the promotion of the separation of particles from the first steps of their creation leading to the particles can not experience a process of growth. From the statistical analysis of the particle's size, the smallest average value was found where the liquid environment is formed by $\mathrm{H}_{2} \mathrm{O}+$ grapheneoxide. Reaching a value that under classical conditions can not be achieved. It is well known that during PLAL synthesis, at lower intensities, there is a greater chance that the fundamental process which leads to detachment would be Coulombian explosion and therefore, lower the size of MNPs. In this context, considering that under the presence of graphene-oxide the solid target would be receiving a low amount of energy, resulting in the production of small particles.

The sample depicted in Figure 7.3, which is irradiated with a similar radiation value that should be hitting the sample in the first experiment, shows that in fact smaller sizes are achieved when the radiation intensity is lowered. Nonetheless, the observation of such small sizes in the first experiment could be not only related to the intensity employed to irradiate the sample, is also probable that bigger particles could be produced but not anchored to the carbonic surface. In order to explore the idea that maybe graphene-oxide sheets were acting as size selectors, particles created with an incoming mean power of $40 \mathrm{~mW}$ in water with an average size of $60 \mathrm{~nm}$ were mixed with a graphene-oxide solution $(1 \mathrm{mg} / \mathrm{ml})$ and stirred for $12 \mathrm{~h}$. The respective micrographs depicted in Figure 7.4 reveals that all the gold NPs have been supported in the graphene-oxide. This supplementary experiment suggests that when a gold disc is irradiated in the presence of graphene-oxide, the tendency of particle's size is to decrease. Nowadays, it has not been reported the fabrication of particles with sizes lower than $6 \mathrm{~nm}$ under classical conditions, without using any chemical stabilizer or capping agent. Since graphene-oxide sheets are present during the whole particles synthesis, and there is a natural tendency for the particles to get anchor onto the graphene's surface, the introduction of the carbonaceous material during the synthesis promotes that primary particles don't agglomerate and grow, acting in this way as a capping agent promoting a the reduction of the final particle's size. 
Therefore, under the same reaction conditions the graphene-oxide has an important influence in lowering the size of the gold NPs.
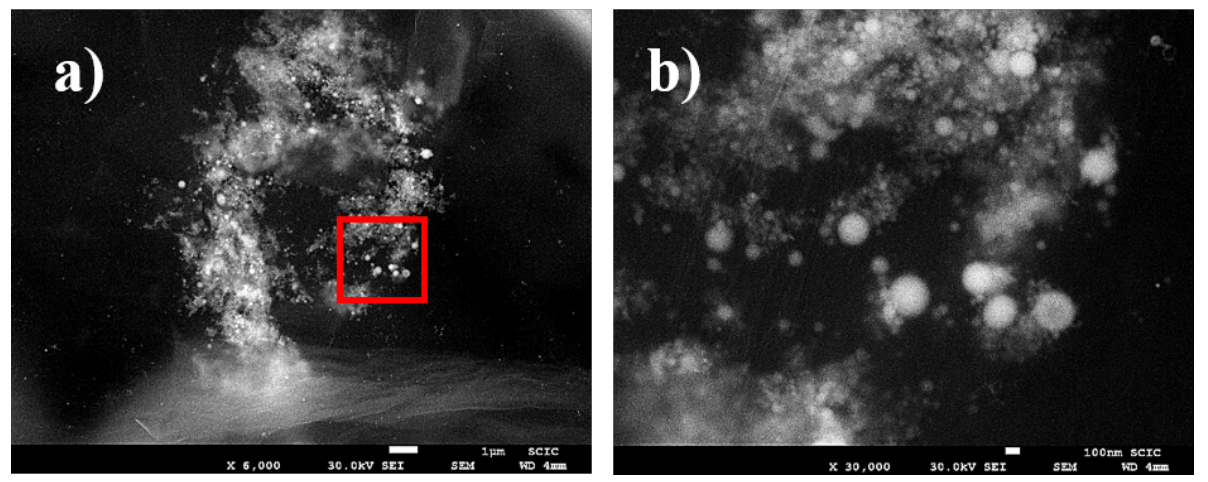

Figure 7.4: Micrograph of Au-NPs hooked in graphene sheets, a) SEM micrograph and b) zoom of the red square in figure a) showing the size of particles clearly bigger than $34 \mathrm{~nm}$.

Moreover, the FS Mapping measurements obtained by means of the EDX system and a special HAADF detector,shows that the PLAL fabrication route produces highly pure Au-NPs anchored on the graphene-oxide sheets in a heterogeneous distribution as can be inferred from Figure 7.5. The FS Mapping results provides information not just about the morphology or size of the particles. As it takes advantage of the EDX system attached to the TEM, the results can provide precise information of the elemental position in the sample, i. e. from which point comes a signal of a certain element. From Figure $7.5 \mathrm{~d}$ ), is clear that there is a concentration of gold NPs in certain areas, and the anchor of particles is not homogeneously distributed. From a statistical analysis, it was determined that the highest concentration of $\mathrm{Au}$ NPs was found where the highest wrinkle concentration was present in the graphene surface. The wrinkles presence is due to crystalline defects in the graphene sheets and the high concentration of NPs in or close to this area can be explained as a combination of electrostatic interactions, overlapping of the extended $\pi$-orbitals of graphene oxide with the $d$-orbitals of the metal NPs and/or allocation of particles at the defects of graphene oxide sheet. However, the electronic singularities promoted by the crystalline defects in a 2-D carbonic structure like graphene-oxide sheets react electronically with the free ligands in metal NP resulting in its immobilization over or close to the defects.

In the other hand, the EDX spectrum shows that when the irradiated area by the electron beam is bigger than particle's size, some extra elements like $\mathrm{Cu}, \mathrm{C}, \mathrm{Cr}$ and $\mathrm{Fe}$ can be detected like in the samples produced in water by classical PLAL conditions 

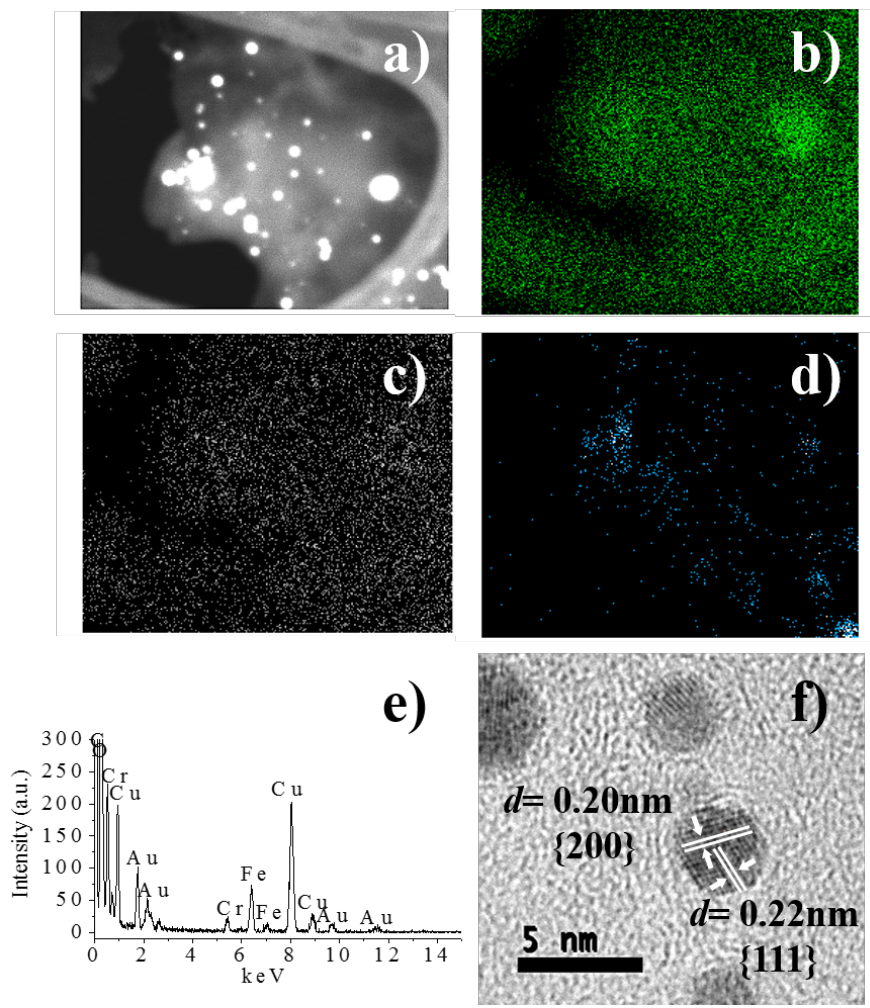

Figure 7.5: FS Mapping Micrographs of gold NPs anchored in Graphene sheets, a) DF-STEM micrograph where the mapping was taken, b) emission of C, c) emission of $\mathrm{O}, \mathrm{d}$ ) emission of $\mathrm{Au}$. e) EDX spectrum of Au-NPs immobilized in GO sample, $\mathrm{f}$ ) HRTEM micrograph of Au-NPs/graphene-oxide showing the $d$ inter-planar spacing.

(Figure 7.6). The presence of copper and carbon is caused by the grid. Fe and $\mathrm{Cr}$ are detected because the microscope objective lenses are made of it. However, the most evident differences between EDX spectrum for samples produced in water without the presence and with the presence of graphene-oxide are the intensities of the peaks that corresponds to carbon and gold. When the particles are anchored to graphenesheets, the peak corresponding to $\mathrm{C}$ increase due to the presence of a large quantity of $\mathrm{C}$ atoms located at the vicinity of the gold NPs, the signal of the gold element is then diminished or masked by the high signal corresponding to $\mathrm{C}$. Therefore, the first logical conclusion that can be extracted from EDX analysis is that there is no evidence of any contamination or byproduct due to we are just observing gold, carbon and the elements forming the TEM's grid or the TEM's objective lenses. As it could be extrapolated from this, the synthesized material seems to be free from any chemical impurity. This argument is ratified by the HRTEM micrographs depicted in Figure 7.6 b), where the lattice fringes indicate the high crystallinity of the material, which allows to determine the $d$ inter-planar spacing that can be represented by 
the $(h k l)$ Miller index. The $d$-spacing of $0.02 n m$ corresponds to the $(200), 0.23 n m$ and $0.24 \mathrm{~nm}$ to the (111) lattice planes of gold respectively. The obtained results are similar to those included in Joint Committee on Powder Diffraction Standards (JCPDS) files for cubic Au crystals (JCPDS: 4-784).

The analysis by HRTEM reveals that the synthesized Au-NPs and Au-NPs/grapheneoxide (Figure $7.5 \mathrm{f}$ )) are free from any other element, chemical impurity or crystalline defect, since the distance between crystallographic planes doesn't present a periodic increase in the $d$ inter-planar spacing or any periodic punctual defect in the atomic arrangement.
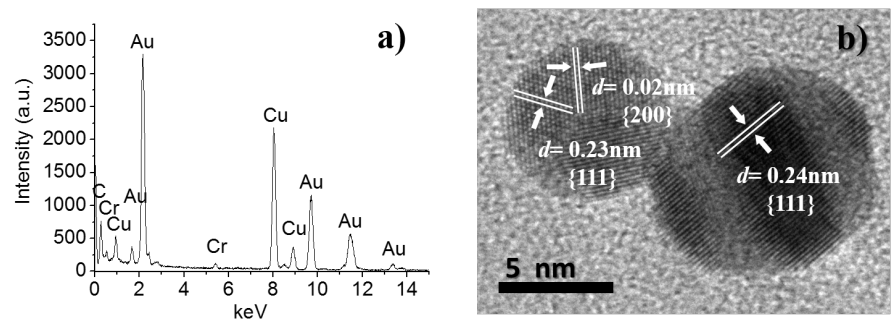

Figure 7.6: a) EDX spectrum of produced gold NPs under classical PLAL conditions, b) HRTEM micrograph of Au-NPs showing the $d$ inter-planar spacing.

However, the elemental analysis and crystallographic analysis of each particle don't provide enough information about the hybrid material all together. For this, is mandatory to perform a crystallographic analysis of the whole sample, MNPs and graphene-oxide sheets before and after the synthesis process. It is evident that the interaction between the light source and the carbonic structure should promote a change in the graphene-oxide sheets, and the presence of MNPs in their surface could promote any other important change in their optical and electronic behavior. In this context, the easiest way to study this changes in the whole nano-composite is through powder X-ray diffraction, since the technique could allow to identify any possible change in the crystalline structure of not only the MNPs, but also of graphene-oxide sheets.

Figure 7.7 displays the XRD patterns of graphene-oxide, reduced graphene-oxide obtained using hydrazine method [248] and Au-NPs/graphene-oxide. The grapheneoxide pattern shows an intense peak at $2 \theta=12^{\circ}$ and a low intensity peak relative to the first one at $42.65^{\circ}$. If we consider that this material preserves the characteristic hexagonal periodic arrangement of carbon atoms presented in the graphite layers, but with some extra oxygen functional groups, the first peak can be associated to the crystalline plane (001), which comes from an increase of the inter-layer distance 
compared to graphite as a consequence of the oxygen functionalities introduced in the carbonaceous material after oxidation [249], the second peak corresponds to the crystalline plane (100) also present in graphite (Miller index and $d$ inter-planar spacing for Hexagonal Graphite are included in Joint Committee on Powder Diffraction Standards (JCPDS: 41-1487)).

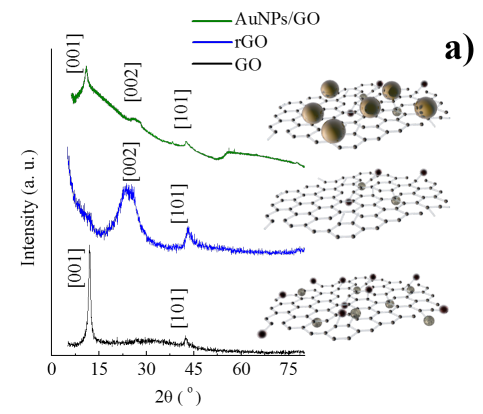

b)

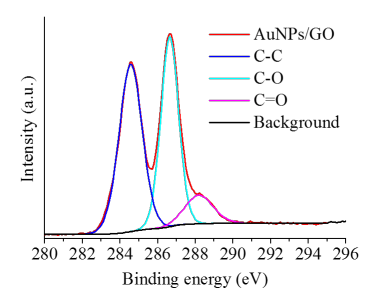

Figure 7.7: XRD patterns of graphene-oxide decorated with ligand-free gold NPs (AuNPs/GO), reduced graphene-oxide by hydrazine method (rGO) and graphene-oxide obtained by the Hummers method (GO).

The reduced graphene-oxide XRD pattern is completely different to the first one, the peak corresponding to (100) disappears but in contrast the peak of the (002) plane appears at $24.85^{\circ}$, and also the peak of (101) is observed at $43.1^{\circ}$. The peak corresponding to (100) disappears because during the reduction of graphene-oxide through hydrazine, many oxygen functional groups are removed from the carbonic structure and part of the $\pi$-network is restored in the material, leading at the same time to the observation of a peak that begins to show a hexagonal structure that comes from graphite. The XRD pattern found for the Au-NPs/graphene-oxide shows an intermediate situation between the two previous diffraction patterns. A peak at $2 \theta=10.85^{\circ}$ corresponding to (001) crystalline plane is observable, also the peak corresponding to (002) and the peak corresponding to (101). The results suggest that laser irradiation has an effect on the graphene material. The presence of the peaks located at (100) and (002), reveal that the laser irradiation is promoting the detachment of oxygen functional groups in the material leading to a partial reduction of graphene-oxide. To ratify the XRD studies, XPS analysis was performed, reveling the presence of $\mathrm{C}-\mathrm{C}, \mathrm{C}-\mathrm{O}$, and $\mathrm{C}=\mathrm{O}$ functional groups that allows us to determine the $\mathrm{C} / \mathrm{O}$ relation in the samples, being 2 (66\% of $\mathrm{C}$ and $33 \%$ of $\mathrm{O}$ ) for GO. $10.4(91.2 \%$ of $\mathrm{C}$ and $8.8 \%$ of $\mathrm{O}$ ) for rGO, and 2.4 (40.6\% of $\mathrm{C}$ and $29.4 \%$ of $\mathrm{O}$ ) for $\mathrm{Au}-\mathrm{NP} / \mathrm{GO}$. These results suggest that laser irradiation has an effect on the graphene material. 
Moreover, the XRD and XPS studies has revealed that laser irradiation results in the partial reduction of GO.

\subsection{Conclusions}

In summary, using a Femtosecond radiation-based synthesis approach, we were able to decorate graphene-oxide sheets with ligand-free Au-NPs and partially reduce the graphene-oxide in a practical single reaction stage avoiding the production of any chemical waste. From a general point of view, the irradiation of a gold disc in water containing a suspension of graphene-oxide leads to the immobilization of the generated ligand-free Au-NPs in the carbonic material surface. The NPs obtained are spherical, heterogeneously distributed along the material, presenting the biggest concentrations in graphene wrinkles and do not tend to aggregate. The presence of graphene-oxide during the synthesis process reduces the average particle size, and the graphene-oxide is partially reduced by its interaction with the laser radiation. We believed that the simplicity and cleanness of the synthesis of Au-NPs/graphene-oxide may inspire future research in the field of materials science and encourage the scientific society to produce future developments of efficient decoration of graphene and related materials based on this approach. 


\section{Chapter 8}

\section{Conclusions}

Nanoscience is envisaged as the next big technological revolution that will affect many of the aspects of human society. Since it has the great potential of improving every single part of human-designed and not human-designed systems, it has answers to solve some of the grand challenges that faces our society as energy production, climate change and health. But to build an environmentally sustainable world, a "green" method to produce, nanostructures and reduce the waste is a necessity. For applications where a low or moderate nanostructure production rate is required, pulse laser ablation in liquids has been envisaged as an alternative to chemistry methods thanks to the simplicity of the procedure and the absence of unnecessary adducts and byproducts. In PLAL, the interaction of the laser radiation with a target immersed in a liquid promotes the extraction of material located at the surface in the form of an ablation plume, which contains atoms, ions and clusters. NPs are formed from the ejected material and they are collected in the liquid as colloids.

The idea of using laser radiation to extract material, in nanometric scale from a solid target immersed in almost any kind of fluid, provides this technique the unique potential to generate clean nanostructures in liquid environments where the conventional nano-fabrication techniques could hardly achieve. The size control, size distribution and efficiency in the formation of the particles is based on radiation parameters, solid target, and liquid environment properties. By choosing the right combination of those properties is possible to even create new materials that don't exist in nature, giving the opportunity to offer useful complex materials that could solve real problems.

The research conducted during this thesis work is focused on two particular problems that deserve a strong scientific effort: The solar power industry efficiency and the development of materials with a combination of outstanding electronic and optical properties. In this context, since the fabrication of metal nanostructures in low 
concentrations in organic oils has been demonstrated to improve their thermal properties, resulting in efficient heat transfer fluids, or even volumetric sunlight absorbers, that promise to transform the solar radiation into consumable electricity. The use of PLAL seems to be ideal to solve the biggest issues of producing metal nanostructures inside organic oils by traditional synthesis ways, that are mostly related to poor stability, production of chemical waste and finally promotion of uncontrolled chemical reactions due to particle purity problems. In the other hand, is well known that assembles composed by nanometric structures are being increasingly used, but up to know the traditional ways to manufacture such kind of novel materials, are based on non environmental friendly techniques that seem to be limited by the basic materials used. Since one of the greatest advantages of PLAL is the possibility to create nanostructures from almost any kind of solid material in liquid environments in a "green" way, this approach seems to be ideal to be used as a basic technique on the production of nanometric assembles.

In particular, the biggest contributions achieved on the present work, lies on the experimental demonstration of the synthesis of gold nanoparticles-based aqueous nanofluids that can be used as both volumetric light absorbers and heat exchangers. The fabrication of novel materials that present a thermal conductivity enhancement of $4.06 \%$ over the most commercial heat transfer fluid, Therminol VP-1 an eutectic mixture of biphenyl and diphenyl oxide, and the best colloidal stability reported in the literature using an organic oil and gold NPs. An finally, the demonstration of partially reduction of graphene-oxide sheets and its decoration with ligand-free Au-NPs in a practical single reaction stage avoiding the production of any chemical waste.

To achieve the as-mentioned contributions, it was mandatory to explore the further modification of synthesized nanostructures by means of using surfactants, and postfragmentation of the already created nanostructures by irradiating the synthesized colloids. The post-processing of the samples lead us to achieve a high control over the size and morphology of the nanostructures. That control resulted into the mitigation of nanostructure agglomeration and ensuring the maximum physical and chemical activity of the materials.

Being more precise, the exploitation of a post-processing stage acquire the biggest importance in the experiments carried out on the scope of harvesting of solar energy. During the creation of nanofluids that could effectively act as both volumetric radiation absorbers and heat transfer fluids, two main characteristics were considered. The first one was the stability of the fluid through the time, considering that any fluid with a potential applicability in the industry should remain for prolonged periods of 
time without any change, it was extremely important that nanostructures contained in the base fluid should keep well distributed in the fluid through the time without clogging. The second one was the morphology of the nanostructures, any solid material in a fluid can promote flow dynamics problems promoting turbulence or any other physical undesired effect. The principal way to face these draw-backs consists in two paths, the fragmentation of already created particles to promote the creation of oxidized nanostructures that don't tend to clog, and the use of surfactants that ensure no agglomeration.

In this context, the use of the laser for fragmenting the material synthesized by PLAL, lead us to obtain a nanofluid (gold NPs in deionized water), able to compete with commercial available nanofluids from a thermal and optical point of view, but showing better benefits such as the spherical shape that can result in better nanofluid motion or the purity of the material. Moreover, when the material undergoes thermal treatment (cycles of heating and cooling), neither the physical nor the chemical characteristics of the nanofluid changes. And its sunlight absorption performance shows a great potential to be used as direct absorber in solar collectors.

When the same experimental approach is used for a liquid ambiance that is not deionized water, gold nanotructures don't get oxidized and tend to agglomerate. Nevertheless is also important to notice that deionized water is not commonly used as a volumetric sunlight absorber or heat transfer fluid in the industry, mainly due to its boiling point. Instead, organic-based oils are preferred but in the counterpart show poor thermal conductivity resulting in slow heat transfer fluids, since metal NPs present a higher thermal conductivity, the introduction of metal NPs in oil-based fluids provides the opportunity to improve their thermal conductivity and therefore their thermal performance. In this sense, gold NPs were created in Therminol VP-1 a commonly used heat transfer fluid. The presence of the metal nanostructures resulted in the enhancement of thermal properties of the base fluid but the particles agglomerate through the time. Post-fragmentation of particles in the colloid mitigate the agglomeration phenomena but not completely, leading to the fabrication of a novel heat transfer fluid with better thermal properties that the commercial available products, that reach a relatively higher stability at the sixth day of room storage.

When a stabilizer agent is used instead of the simple post-fragmentation of particles, a superior mitigation of the agglomeration phenomenon is achieved. In fact, by the use of an ionic surfactant that is not degrading at temperature values under $200^{\circ} \mathrm{C}$ (tetra- $n$-octylammonium bromide $\left(\left(C_{8} H_{17}\right)_{4} N B r\right.$, TOAB, $\left.>98 \%\right)$ ), it has been possible to fabricate the most stable nanofluid composed by Therminol VP-1 
and gold NPS, reported up to now. In counterpart, the use of any chemical additive may lead to a decrease in the thermal enhancement or creation of by-products that eventually could result into the further modification of the physical and chemical characteristics of the created material. When the chemical additive is used during the PLAL and post-fragmentation process, the chemical reactions between the base fluid, the metal NPs and the ionic surfactant seem not to be greater than the correct functionalization of metal NPs with the surfactant. In this sense, no further modifications of the material can be expected but just a significant lower in the thermal performance of the final material. The stabilization of the material by the simple addition of the surfactant to the base liquid during the laser-based synthesis process, reveals the great possibility to functionalize materials by PLAL in a non-complex way. In this context, the exploitation of all of the achievements reached during this thesis work culminated with the creation of a nano-assemble composed by gold NPS and a material which electronic outstanding properties that led it to be considered the material of the future, graphene.

The synthesis of such material was achieved by anchoring ligand-free gold NPs on the surface of partially reduced graphene-oxide sheets, resulting from the irradiation of a femtosecond pulsed laser in a solid gold target immersed in a solution containing graphene-oxide sheets dispersed in de-ionized water. The irradiation of the solid target with a high power laser promotes the ablation of it, when the environment surrounding the process is water, metallic material detached from the target tends to get oxide leading to metal NPs with a ligand-free surface, able to chemically interact with other elements. If additionally graphene-oxide sheets are present during the irradiation process, the high power radiation of the pulsed laser can promote the reduction of the carbonic material by taking out oxygen functional groups and part of the $\pi$-network can be restored in the material, leading to the fabrication of gold NPs and reduction of graphene-oxide sheets at the same time. Moreover, the presence of graphene-oxide during the synthesis process reduces the average particle size, and the particles are immobilized in the reduced graphene-oxide surface due to the carbonic material acts as a capping agent during the PLAL process resulting in the functionalization of metal NPs with a periodically arranged carbon matrix. From a general point of view, this thesis work represents an experimental proof that using PLAL is possibility to create nanomaterials from metal solid targets with almost any desired size and size distribution in at least organic oils and solutions containing deionized water and graphene oxide sheets. The results presented suggest that the employed synthesis method represents an advantage over conventional methods 
to produce metal NPs in heat transfer fluids that may potentially have a significant impact on the improvement of harvesting efficiency of solar light with nanofluids, and in graphene-based dispersions that may encourage the scientific society to produce future developments of efficient decoration of graphene and related materials based on this approach.

The proven versatility and efficiency of PLAL, makes this technique a possible solution for multi-disciplinary projects where a nanostructure fabrication technique is required to be used in complex colloidal ambiances, and even when PLAL is still a young nanostructure synthesis approach and it requires more research to understand each physical and chemical phenomenon associated with their use. We strongly believe that the simplicity and cleanness of PLAL represents a synthesis alternative that may inspire future research in a wide range of fields, from materials science, to life science.

\section{Future scope of the investigation efforts.}

PLAL have opened for our research group an exciting and fascinating research line that is step by step taking us to dabble in different fields and collaborate with multidisciplinary research teams. Based on this premise, we strongly believe that is time for our research group to try to venture in the bio-medicine world, and take a step further on the harvesting of solar light field.

Experiments performed during the whole thesis work have demonstrated that the functionalization of metal NPs is possible and easy when PLAL is used. It is only necessary that the chemical agent used to functionalize, be dispersed in the liquid environment when the nanostructures are synthesized using the laser radiation. The natural tendency of the chemical agents will be acting as a capping agent as it was described in the Chapter 2 and the final nanostructure will be linked to molecules with potential biological or chemical activity. The experimental step that seems obvious is to funtionalize gold NPs with not so complex molecules that provide a chemical response.

Moreover, the production of nanostructures from different solid targets has brought the teaching that, although gold is a material having great optical properties, is not the material that can present the best performance as a volumetric light absorber. Further investigation in these directions are being performed. 


\section{Resumen en español}

\section{Objetivos de la investigación realizada}

En la actualidad, la nanociencia y nanotecnología están llevando a la humanidad a un nivel de vida que hasta hace unos años sólo podía ser imaginado, presentando soluciones y mejoras sustanciales a problemas de la industria, de la medicina y de la vida diaria.

La nanotecnología usa como bloques básicos, estructuras con tamaños menores a 100 nanómetros, la mil millonésima fracción de un metro. Con estos bloques fundamentales se pretende crear dispositivos a escalas moleculares o incluso atómicas. La gran ventaja de crear instrumentos tan pequeños radica en que se pueden llevar a cabo procesos controlados a escalas temporales y espaciales en las que se encuentran los átomos. Con ello, se pueden controlar procesos secuenciales que pueden llegar a ser muy complejos. No obstante, esa no es la única ventaja, los materiales estructurados a escalas tan pequeñas tienden a presentar propiedades y comportamientos diferentes a los observados en materiales macroscópicamente ordenados. Esto debido a que las nano-estructuras, generalmente muestran diferentes propiedades químicas y físicas que dependen del tamaño. Esto desde luego abre la posibilidad de diseñar y fabricar dispositivos nunca antes imaginados con materiales que presentan características que nunca antes habían sido observadas.

La manera para obtener dichos materiales, responde a dos vertientes principalmente. La vertiente que toma en cuenta la fabricación de estas estructuras desde sus elementos fundamentales hasta crecer estructuras que alcancen dichos tamaños (vertiente bottom-up), y la vertiente que toma un material macroscópico y lo fracciona hasta obtener piezas de dicho material que alcanzan los nanómetros (vertiente top-down). No obstante, sin importar que vertiente se utilice, aún existen campos del conocimiento que requieren la incursión plena de la nanotecnología, pero esta sigue limitada precisamente por los métodos de fabricación. En este contexto, 
existen variados campos en los que se utilizan materiales que hasta la fecha no se han podido estructurar a escalas nanométricas o bien no ha sido posible generar nano-estructuras dentro de ellos. Adicionalmente la mera construcción o inducción de una estructura a dichas escalas implica para los métodos convencionales la generación de productos químicos indeseados que posteriormente pueden llevar a la aparición de efectos que o bien no se pueden controlar, o que realmente afectan la funcionalidad del nano-material.

Para afrontar dichos problemas, se ha propuesto el uso de una técnica que combina elementos top-down y bottom-up que promete generar estructuras nanométricas sin crear residuos químicos indeseados, de casi cualquier sólido macroscópico en casi cualquier medio líquido facilitando la generación de estructuras nanométricas cada vez más complejas, Ablación por Láser Pulsado en Líquidos o PLAL por sus siglas en inglés.

El objetivo central de esta tesis es la implementación de dicha técnica en la Universitat Jaume I., en explorar el uso de láseres de pulsos ultra cortos para llevar a cabo dicha técnica, y explorar posibles modificaciones a la técnica original para con ello ser capaces de crear coloides nano-estructurados que puedan resolver problemas reales existentes en el campo de la energía, y finalmente crear nuevas estructuras manométricas complejas que tengan potenciales aplicaciones en campos como la optoelectrónica o bio-medicina.

\section{Planteamiento y aportaciones originales}

Es bien sabido que una gran alternativa al uso de los combustibles fósiles es la explotación de energía solar, y para ello existen muchos dispositivos capaces de alcanzar grandes eficiencias, sin embargo, el dispositivo que promete llegar a una gran cantidad de personas es el acumulador de energía solar. Generalmente estos dispositivos se componen de una gran torre que contiene un líquido de transferencia de calor, el cual es llevado a la punta de la torre y calentado gracias a que cientos de espejos hiperbólicos concentran la radiación solar en dicha zona. Una vez calentado este es desplazado a una trampa fría para generar vapor y posteriormente mover turbinas que generarán electricidad. La gran mayoría de estos líquidos de transferencia de calor presentan conductividades térmicas muy bajas y el proceso de calentamiento puede llegar a durar varias horas, 
sin mencionar que durante su traslado de la punta de la torre a cualquier zona requiere una pérdida de calor. Para resolver este problema, en este trabajo de investigación se exploró la posibilidad de usar dicho fluido como un absorbedor volumétrico de calor y un líquido de transferencia de calor al mismo tiempo dispersando nanoestructuras con propiedades ópticas importantes en el líquido de transferencia de calor. Esta idea no es nueva ya que varios grupos la han llevado a cabo, pero la aportación que pudimos lograr fue demostrar que es posible usar PLAL para generar nanoestructuras que pueden absorber radiación solar en un amplio rango en un medio líquido que puede ser usado como fluido de transferencia de calor.

Muchos trabajos de investigación dirigidos en esta línea se pueden encontrar con grandes dificultades al trasladarse de sistemas que pueden ser fácilmente controlados en el laboratorio a sistemas que se usan en la industria real. Por ello decidimos utilizar un líquido de transferencia de calor utilizado en plantas concentradoras de energía solar reales con una baja conductividad térmica, Therminol VP-1, o bien una mezcla eutéctica de bifenilo y oxido de difenilo. La generación de partículas metálicas en este líquido promueve un incremento en su conductividad térmica y ya se había demostrado por Wang et. al. [208], no obstante sus resultados demostraron que el fluido obtenido no era estable a lo largo del tiempo siendo poco viable su utilización en el mercado real. Nuestra aportación consistió en utilizar PLAL para generar dicho fluido, la hipótesis que se perseguía estaba ligada a que los residuos químicos propios de la técnica de fabricación de nano-estructuras usada por Wang debieron de promover dicha inestabilidad en el fluido. Los resultados demostraron que de hecho era cierto, los residuos químicos derivados del método de fabricación sí influyen en la estabilidad del fluido a través del tiempo llevando a la obtención de un fluido más estable gracias a la utilización de una técnica que no promueve la generación de residuos químicos.

Siguiendo con la línea de investigación desarrollada, el siguiente objetivo planteado por el grupo fue generar un fluido con una conductividad térmica mejorada que pudiera ser usado en aplicaciones reales. Para ello se trabajó con el mismo líquido de transferencia de calor, pero aprovechando la versatilidad de PLAL, se exploró la posibilidad de funcionalizar los 
nano-materiales creados para alcanzar un grado de estabilidad idóneo para la industria. En este sentido, se funcionalizaron las nano-estructuras metálicas generadas por PLAL, con un surfactante iónico conocido popularmente como TOAB. Dicho surfactante se degrada a temperaturas mayores a $200 C^{\circ}$ y promueve una repulsión estérica en aceites orgánicos, ambas características necesarias para estabilizar partículas metálicas en Therminol VP-1. Los resultados demostraron que no solo había tenido éxito la funcionalización, sino que adicionalmente se había conseguido el objetivo, gracias a PLAL se creó el nanofluido compuesto por nanopartículas de oro y Therminol VP-1 más estable reportado hasta ahora en la literatura.

Tras adquirir experiencia haciendo uso de PLAL para generar estructuras con tamaños nanométricos en medios líquidos en donde el hacerlo por vías convencionales resulta muy complejo o imposible. Decidimos dar el siguiente paso generando estructuras nanométricas mucho más complejas. En este sentido, elegimos diseñar un nano-compuesto, es decir, un material que estuviese compuesto por más de un material estructurado a escala nanométrica. La elección de ambos materiales se centró en su potencial para ser usado en diversas áreas tecnológicas. Por un lado se usó oro, ya que presenta importantes propiedades ópticas cuando es reducido a la nano-escala y por otro lado se eligió grafeno ya que es un material bidimensional que presenta importantes características electrónicas y la conjunción de ambas propiedades podría llevarnos a obtener un material que podría ser usado en diversos campos. Para la generación de dicho nano-compuesto se sintetizaron nanopartículas de oro en una solución de hojas de grafeno dispersas en agua haciendo uso de PLAL. Los resultados demostraron que las hojas de grafeno estuvieron actuando como agente funcionalizador durante PLAL promoviendo que las nanoestructuras metálicas se anclar en las hojas de grafeno. Adicionalmente, la interacción entre el grafeno y la radiación láser llevaron a la reducción del grafeno, siendo el grafeno reducido una estructura carbónica que puede presentar propiedades súper-conductoras.

En suma, el uso de PLAL para la fabricación de dicho nano-compuesto no solamente fue exitosa, sino que además demostró ser útil para generar un complejo material nanoestructurado con propiedades que merecen estudios adicionales. 
Durante la realización de cada uno de los trabajos de investigación derivados de la implementación de PLAL para generar estructuras manométricas con posibles aplicaciones en la nanotecnología, se exploraron modificaciones en la forma básica del aparato experimental usado para PLAL, llevando a la exploración del uso de pulsos de femtosegundos y a la utilización de un subsecuente paso para procesar el material obtenido en PLAL. Dicho paso consistió en la fragmentación de nano-materiales previamente creados por PLAL haciendo uso del láser, para así controlar su tamaño final. Adicionalmente se exploró la utilización de surfactantes iónicos y hojas de grafeno para funcionalizar el nano-material sintetizado por PLAL.

\section{Conclusiones}

En este trabajo de tesis, mediante PLAL se sintetizaron nano-ensambles basados en metal con potenciales aplicaciones tecnológicas en el campo de la recolección de la luz solar, la catálisis, la biomedicina y la optoelectrónica. Una estrategia basada en radiación láser que promete ser utilizada como una ruta de fabricación estándar en los campos donde se requiere que nano-estructuras altamente puras y químicamente activas, sean creadas en medios líquidos no convencionales, a escalas no masivas. Se estudiaron parámetros del láser, diferentes soluciones y solutos para controlar la morfología, tamaño, distribución de tamaño, la estabilidad coloidal, respuesta térmica y óptica de las muestras.

Las mayores contribuciones del presente trabajo radican en la demostración experimental de la síntesis de nanofluidos basados en nanopartículas de oro que se puede utilizar tanto como absorbentes de luz volumétricos, como intercambiadores de calor. La fabricación de nuevos materiales que presentan una mejora de la conductividad térmica de 4,06\% sobre el fluido de transferencia de calor comercial más utilizado en concentradores de radiación solar, Therminol VP-1 siendo una mezcla eutéctica de bifenilo y de óxido de difenilo, y la mejor estabilidad coloidal reportada en la literatura utilizando dicho aceite orgánico y nanopartículas de oro. Por último, la demostración experimental de la reducción de grafeno y su decoración con nanopartículas de oro en una sola y práctica reacción evitando la producción de residuos químicos. 
Para lograr las contribuciones antes mencionadas, fue preciso explorar la modificación adicional de nanoestructuras sintetizadas mediante el uso de surfactantes, y etapas de post-fragmentación de las nanoestructuras obtenidas por PLAL mediante su irradiación. la modificación adicional de las muestras nos llevó a alcanzar un alto control sobre el tamaño y la morfología de las nanoestructuras. Dicho control llevó a la mitigación de la aglomeración de nanoestructuras en los diferentes medios líquidos, garantizando la máxima actividad física y química de los materiales.

Desde un punto de vista general, este trabajo de tesis representa una prueba experimental de que el uso de PLAL posibilita la creación de nanomateriales con casi cualquier tamaño deseado y distribución de tamaño, a partir de sólidos metálicos en líquidos orgánicos y soluciones compuestas por agua y hojas de grafeno, sin producir subproductos o cualquier reacción química cruzada no deseada. Los resultados presentados sugieren que el método de síntesis empleado representa una ventaja sobre los métodos convencionales para producir nano-estructuras de metales en fluidos de transferencia de calor que potencialmente pueden tener un impacto significativo en la mejora de la eficiencia de la recolección de radiación solar y en dispersiones basadas en grafeno, alentando asá la sociedad científica para producir futuros desarrollos en la decoración eficiente de grafeno y materiales relacionados basados en este enfoque. La probada versatilidad y la eficiencia de PLAL, hace de esta técnica una posible solución para proyectos multidisciplinares donde se requiere una técnica de fabricación de nano-estructuras que pueda ser implementada en ambientes coloidales complejos, e incluso cuando PLAL sigue siendo una técnica de síntesis de nanoestructuras joven y requiere más investigación para entender cada fenómeno físico y químico asociado con su uso, creemos firmemente que la sencillez y la pureza de PLAL representa una alternativa de síntesis que puede inspirar a futuras investigaciones en una amplia gama de campos, desde la ciencia de los materiales, hasta ciencias de la vida. 


\section{Bibliography}

[1] R. Torres-Mendieta, R. Mondragon, E. Julia, O. Mendoza-Yero, J. Lancis, and G. Minguez-Vega. Fabrication of high stable gold nanofluid by pulsed laser ablation in liquids. Advanced Materials Letters, 6:1037-1042, 2015.

[2] R. Torres-Mendieta, R. Mondragon, E. Julia, O. Mendoza-Yero, E. Cordoncillo, J. Lancis, and G. Minguez-Vega. Fabrication of gold nanoparticles in Therminol VP-1 by laser ablation and fragmentation with fs pulses. Laser Physics Letters, 11:126001, 2014.

[3] International Year of Light. Ibn al-haytham and the legacy of arabic optics. urlhttp://www.light2015.org/, 2015.

[4] Tabak John. Mathematics and the laws of nature: developing the language of science. Facts On File, Inc., New York, USA, 2004.

[5] Devlin Keith. The Language of Mathematics: Making the Invisible Visible. Henry Holt and Company, New York, USA, 2000.

[6] Cornell University. Lecture 2 - the relation of mathematics to physics, the character of physical law, richard feynman. urlhttp://www.cornell.edu/, 2015.

[7] H. Crew, C. Huygens, T. Young, A.J. Fresnel, and F. Arago. The Wave Theory of Light: Memoirs of Huygens, Young and Fresnel. Number v. 15 in Scientific memoirs. American Book Company, 1900.

[8] J. C. Maxwell. A dynamical Theory of the Electromagnetic Field. Proceedings of the Royal Society of London, 13:531-537, 1865.

[9] A. Einstein. Uber einen die Erzeugung und Verwandlung des Lichtes betreffenden heuristischen Gesichtspunkt. Annalen der Physik, 322:132-148, 1905.

[10] E Hecht and A Zajac. Optics. Addison-Wesley, Massachusetts, USA, 2001. 
[11] T.H. Maiman. Ruby laser systems, nov 1967. US Patent 3,353,115.

[12] N. Bohr. Atomic Structure. Nature, 107:104-107, 1921.

[13] P. Franken, A. Hill, C. Peters, and G. Weinreich. Generation of Optical Harmonics. Physical Review Letters, 7:118-119, 1961.

[14] Rachid Ladj, Thibaud Magouroux, Mohamed Eissa, Marc Dubled, Yannick Mugnier, Ronan Le Dantec, Christine Galez, Jean-Pierre Valour, Hatem Fessi, and Abdelhamid Elaissari. Aminodextran-coated potassium niobate (knbo3) nanocrystals for second harmonic bio-imaging. Colloids and Surfaces A: Physicochemical and Engineering Aspects, 439:131 - 137, 2013.

[15] He Zhang, Lian Liu, Xin Fu, and Zhenjun Zhu. Microfluidic beads-based immunosensor for sensitive detection of cancer biomarker proteins using multienzyme-nanoparticle amplification and quantum dotslabels. Biosensors and Bioelectronics, 42:23 - 30, 2013.

[16] Zhaohui Li, Ying Wang, Jun Wang, Zhiwen Tang, Joel G. Pounds, and Yuehe Lin. Rapid and sensitive detection of protein biomarker using a portable fluorescence biosensor based on quantum dots and a lateral flow test strip. Analytical Chemistry, 82:7008 - 7014, 2010.

[17] N. V. Karlov. Laser-induced chemical reactions. Applied Optics, 13:301 - 309, 1974.

[18] N. V. Karlov, Y. N. Petrov, A. M. Prokhoro, and Stelmakh O. M. Dissociation of boron trichloride molecules by co2 laser radiation. JETP Letters-USSR, 11:135, 1970 .

[19] S.D. Rockwood and J.W. Hudson. Laser driven synthesis of bhcl2 from bcl3 and h2. Chemical Physics Letters, 34:542 - 545, 1975.

[20] R. Brinkmann. Microsecond range laser pulse generator - has nonlinear crystal in laser resonator and has pulse extending unit to determine pulse shape and period, Jul 27 1995. DE4401917-A1.

[21] D. G. Ball, D. L. Birx, E. G. Cook, and J. L. Miller. Magnetic modulator for copper vapour laser - comprises magnetic compression circuit for inverting series of electrical pulses from microsecond to nanosecond pulse width range and step-up transformer, Dec 15 1993. GB2267790-A. 
[22] Carsten Framme, Andreas Walter, Philipp Prahs, Dirk Theisen-Kunde, and Ralf Brinkmann. Comparison of threshold irradiances and online dosimetry for selective retina treatment (srt) in patients treated with 200 nanoseconds and 1.7 microseconds laser pulses. Lasers in Surgery and Medicine, 40:616-624, 2008.

[23] John P.M. Wood, Malcolm Plunkett, Victor Previn, Glyn Chidlow, and Robert J. Casson. Nanosecond pulse lasers for retinal applications. Lasers in Surgery and Medicine, 43:499-510, 2011.

[24] B. Chichkov. Femtosecond, picosecond and nanosecond laser ablation of solids. Applied physics. A, Materials science and processing, 63:109-115, 1996.

[25] Thomas J. Dougherty, Charles J. Gomer, Barbara W. Henderson, Giulio Jori, David Kessel, Mladen Korbelik, Johan Moan, and Qian Peng. Photodynamic therapy. Journal of the National Cancer Institute, 90:889-905, 1998.

[26] Charles Kittel. Introduction to Solid State Physics. John Wiley and Sons, Inc., USA, 2005.

[27] Zhuo Li, Jing Li, Yanyan Zhu, Xiangjun Tian, and Huaming Wang. Variant selection in laser melting deposited alpha plus beta titanium alloy. Journal of Alloys and Compounds, 661:126 - 135, 2016.

[28] Andrew J. Pinkerton and Lin Li. Multiple-layer laser deposition of steel components using gas- and water-atomised powders: the differences and the mechanisms leading to them. Applied Surface Science, 247:175 - 181, 2005.

[29] Isak I. Beilis. Laser plasma generation and plasma interaction with ablative target. Laser and Particle Beams, 25:53-63, 2007.

[30] Y. Hu. Increasing the capability of laser peen forming to bend titanium alloy sheets with laser-assisted local heating. Materials and design, 90:364-372, 2016.

[31] R. R. Montés-Micó. Femtosecond laser versus mechanical keratome lasik for myopia. Ophthalmology, 114:62-68, 2007.

[32] K. Sato, Osanai. H., and A. Kawamura. Laser cutting machine - with the projection of the centre of gravity of the cutter arm located between the rails carrying this arm, Mar 30 1995. DE4434504-A1. 
[33] M. Liam. Machining performance of laser surface micro-textured drilling tools. International journal of surface science and engineering, 5:98-115, 2011.

[34] K. Howard. Welding aluminum sheet using a high-power diode laser - a look at fillet welds in lap joints of aluminum alloy 5182. Welding journal, 85:101S-110S, 2006 .

[35] Moonju Cho, M. Aoulaiche, R. Degraeve, C. Ortolland, T. Kauerauf, B. Kaczer, P. Roussel, T.Y. Hoffmann, and G. Groeseneken. Interface/bulk trap recovery after submelt laser anneal and the impact to nbti reliability. Electron Device Letters, IEEE, 31:606-608, 2010.

[36] Esther Rebollar, Margarita Hernández, Mikel Sanz, Susana Pérez, Tiberio A. Ezquerra, and Marta Castillejo. Laser-induced surface structures on gold-coated polymers: Influence of morphology on surface-enhanced raman scattering enhancement. Journal of Applied Polymer Science, 132:n/a-n/a, 2015.

[37] Alfred Vogel, S. Busch, K. Jungnickel, and R. Bringruber. Mechanisms of intraocular photodisruption with picosecond and nanosecond laser pulses. Lasers in surgery and medicine, 15:32-43, 1994.

[38] J. Turkevich and G. Kim. Palladium: preparation and catalytic properties of particles of uniform size. Science, 169:873-879, 1970.

[39] Quazi T. H. Shubhra, Tivadar Feczkó, Andrea F. Kardos, Judit Tóth, Hana Mackova, Daniel Horak, Gyorgy Dósa, and János Gyenis. Co-encapsulation of human serum albumin and superparamagnetic iron oxide in plga nanoparticles: Part ii. effect of process variables on protein model drug encapsulation efficiency. Journal of Microencapsulation, 31:156-165, 2014.

[40] Csaba László, Ramin Sattari, Boris Chichkov, and Stephan Barcikowski. Gram scale synthesis of pure ceramic nanoparticles by laser ablation in liquid. Journal of Physical Chemistry C, 114:2421-2427, 2010.

[41] Chiara Daraio and Sungho Jin. Nanotechnology for Biology and Medicine: At the Building Block Level, chapter Synthesis and Patterning Methods for Nanostructures Useful for Biological Applications, pages 27-44. Springer New York, New York, NY, 2012. 
[42] James J. Norman and Tejal A. Desai. Methods for fabrication of nanoscale topography for tissue engineering scaffolds. Annals of Biomedical Engineering, 34:89-101, 2006.

[43] Shinji Okazaki. High resolution optical lithography or high throughput electron beam lithography: The technical struggle from the micro to the nano-fabrication evolution. Microelectronic Engineering, 133:23 - 35, 2015.

[44] Chang Yao. Anodization: a promising nano-modification technique of titanium implants for orthopedic applications. Journal of nanoscience and nanotechnology, 6:2682-2692, 2006.

[45] P. H. Yih, V. Saxena, and A. J. Steckl. A review of sic reactive ion etching in fluorinated plasmas. physica status solidi (b), 202:605-642, 1997.

[46] Vincent S. Smentkowski. Trends in sputtering. Progress in Surface Science, $64: 1-58,2000$.

[47] Alfred Vogel and Vasan Venugopalan. Mechanisms of pulsed laser ablation of biological tissues. Chemical Reviews, 103:577-644, 2003.

[48] Nathan Blow. Nanotechnology: Could it be a small world after all? Nature, 452:901-4, 2008.

[49] Daniele Ruggiu. Temporal perspectives of the nanotechnological challenge to regulation: How human rights can contribute to the present and future of nanotechnologies. Nanoethics, 7:201-215, 2013.

[50] Ed Gerstner. Nanotechnology goes large. Nature, 425:244, 2003.

[51] C. B. Murray, C. R. Kagan, and M. G. Bawendi. Synthesis and characterization of monodisperse nanocrystals and close-packed nanocrystal assemblies. Annual Review of Materials Science, 30:545-610, 2000.

[52] Jun-Ling Guo, Yao-De Chiou, Wen-I Liang, Heng-Jui Liu, Ying-Jiun Chen, Wei-Cheng Kuo, Chih-Ya Tsai, Kai-An Tsai, Ho-Hung Kuo, Wen-Feng Hsieh, Jenh-Yih Juang, Yung-Jung Hsu, Hong-Ji Lin, Chien-Te Chen, Xue-Pin Liao, Bi Shi, and Ying-Hao Chu. Complex oxide--noble metal conjugated nanoparticles. Advanced Materials, 25:2040-2044, 2013. 
[53] M.A. Gondal, A.M. Ilyas, T.A. Fasasi, M.A. Dastageer, Z.S. Seddigi, T.F. Qahtan, M. Faiz, and G.D. Khattak. Synthesis of green tio2/zno/cds hybrid nanocatalyst for efficient light harvesting using an elegant pulsed laser ablation in liquids method. Applied Surface Science, 357, Part B:2217 - 2222, 2015.

[54] Annette Barchanski, Dominik Funk, Olga Wittich, Christoph Tegenkamp, Boris N. Chichkov, and Csaba L. Sajti. Picosecond laser fabrication of functional gold-antibody nanoconjugates for biomedical applications. The Journal of Physical Chemistry C, 119:9524-9533, 2015.

[55] Stephan Link and Mostafa A. El-Sayed. Spectral properties and relaxation dynamics of surface plasmon electronic oscillations in gold and silver nanodots and nanorods. The Journal of Physical Chemistry B, 103:8410-8426, 1999.

[56] Stephan Link and Mostafa A. El-Sayed. Shape and size dependence of radiative, non-radiative and photothermal properties of gold nanocrystals. International reviews in physical chemistry, 19:409-453, 2000.

[57] Delia J. Milliron, Steven M. Hughes, Yi Cui, Liberato Manna, and et al. Colloidal nanocrystal heterostructures with linear and branched topology. Nature, 430:190-5, 2004.

[58] Stephan Link, , and Mostafa A. El-Sayed. Optical properties and ultrafast dynamics of metallic nanocrystals. Annual Review of Physical Chemistry, 54:331366, 2003.

[59] Youngblood W. Justin, Lee Seung-Hyun Anna, Maeda Kazuhiko, and Mallouk Thomas E. Visible light water splitting using dye-sensitized oxide semiconductors. Accounts of Chemical Research, 42:1966-1973, 2009.

[60] Ivan O. Sosa, Cecila Noguez, and Ruben G. Barrera. Optical properties of metal nanoparticles with arbitrary shapes. The Journal of Physical Chemistry B, 107(26):6269-6275, 2003.

[61] G.W. Yang. Laser ablation in liquids: Applications in the synthesis of nanocrystals. Progress in Materials Science, 52(4):648 - 698, 2007.

[62] Guofeng Wang, Qing Peng, and Yadong Li. Lanthanide-doped nanocrystals: Synthesis, optical-magnetic properties, and applications. Accounts of Chemical Research, 44:322-332, 2011. 
[63] Fumitaka Mafuné, Jun ya Kohno, Yoshihiro Takeda, , Tamotsu Kondow, and Hisahiro Sawabe. Formation of gold nanoparticles by laser ablation in aqueous solution of surfactant. The Journal of Physical Chemistry B, 105:5114-5120, 2001.

[64] N. Wang, Y. Cai, and R.Q. Zhang. Growth of nanowires. Materials Science and Engineering: R: Reports, 60:1 - 51, 2008.

[65] John P. McGahan and Vanessa A. Raalte. Tumor Ablation: Principles and Practice, chapter History of Ablation, pages 3-16. Springer New York, New York, NY, 2005.

[66] SI Dolgaev, AA Lyalin, AV Simakin, and GA Shafeev. Laser ablation in a liquid-medium as a technique for solid sampling. Journal of analytical atomic spectrometry, 6:541-544, 1991.

[67] P. P. Patil, D. M. Phase, S. A. Kulkarni, S. V. Ghaisas, S. K. Kulkarni, S. M. Kanetkar, S. B. Ogale, and V. G. Bhide. Pulsed-laser induced reactive quenching at liquid-solid interface: Aqueous oxidation of iron. Phys. Rev. Lett., 58:238$241,1987$.

[68] John Neddersen, George Chumanov, and Therese M. Cotton. Laser ablation of metals: A new method for preparing sers active colloids. Appl. Spectrosc., 47:1959-1964, 1993.

[69] James D. Blakemore, Harry B. Gray, Jay R. Winkler, and Astrid M. Muller. Co3o4 nanoparticle water-oxidation catalysts made by pulsed-laser ablation in liquids. ACS Catalysis, 3:2497-2500, 2013.

[70] S Di Bucchianico, L Migliore, P Marsili, and C Vergari. Cyto- and genotoxicity assessment of gold nanoparticles obtained by laser ablation in a549 lung adenocarcinoma cells. Journal of Nanoparticle Research, 17:213, 2015.

[71] Mohammd Hossein Mahdieh and Behzad Fattahi. Effects of water depth and laser pulse numbers on size properties of colloidal nanoparticles prepared by nanosecond pulsed laser ablation in liquid. Optics and Laser Technology, 75:188 $-196,2015$.

[72] Ma Rory, R. Amarantha, and Kim Tae Kyu. Effects of laser energy density on size and morphology of nio nanoparticles prepared by pulsed laser ablation in liquid. Bulletin of the Korean Chemical Society, 36:5 - 6, 2015. 
[73] Csaba László Sajti, Svea Petersen, Ana Menéndez-Manjón, and Stephan Barcikowski. In-situ bioconjugation in stationary media and in liquid flow by femtosecond laser ablation. Applied Physics A, 101:259-264, 2010.

[74] V. Lazic and S. Jovićević. Laser induced breakdown spectroscopy inside liquids: Processes and analytical aspects. Spectrochimica Acta Part B: Atomic Spectroscopy, 101:288 - 311, 2014.

[75] R.A. Ganeev, M. Baba, A.I. Ryasnyansky, M. Suzuki, and H. Kuroda. Characterization of optical and nonlinear optical properties of silver nanoparticles prepared by laser ablation in various liquids. Optics Communications, 240:437 $-448,2004$.

[76] Danny Perez, Laurent Karim Béland, Delphine Deryng, Laurent J. Lewis, and Michel Meunier. Numerical study of the thermal ablation of wet solids by ultrashort laser pulses. Phys. Rev. B, 77:014108, 2008.

[77] R. Fabbro, J. Fournier, P. Ballard, D. Devaux, and J. Virmont. Physical study of laser-produced plasma in confined geometry. Journal of applied physics, 68:775-784, 1990 .

[78] Carsten Momma, Boris N Chichkov, Stefan Nolte, Ferdinand von Alvensleben, Andreas Tunnermann, Herbert Welling, and Bernd Wellegehausen. Short-pulse laser ablation of solid targets. Optics Communications, 129:134 - 142, 1996.

[79] D. Perez and L. Lewis. Molecular-dynamics study of ablation of solids under femtosecond laser pulses. Physical review. B, Condensed matter, 67:184102, 2003.

[80] A. Miotello and R. Kelly. Laser-induced phase explosion: new physical problems when a condensed phase approaches the thermodynamic critical temperature. Applied Physics A, 69:S67-S73, 1999.

[81] Patrick Lorazo, Laurent J. Lewis, and Michel Meunier. Short-pulse laser ablation of solids: From phase explosion to fragmentation. Phys. Rev. Lett., 91:225502, 2003.

[82] Patrick Lorazo, Laurent J. Lewis, and Michel Meunier. Thermodynamic pathways to melting, ablation, and solidification in absorbing solids under pulsed laser irradiation. Phys. Rev. B, 73:134108, 2006. 
[83] Yuji Sano, Naruhiko Mukai, Koki Okazaki, and Minoru Obata. Residual stress improvement in metal surface by underwater laser irradiation. Nuclear Instruments and Methods in Physics Research Section B: Beam Interactions with Materials and Atoms, 121:432 - 436, 1997.

[84] Takeshi Tsuji, D.-H. Thang, Yuuki Okazaki, Masataka Nakanishi, Yasuyuki Tsuboi, and Masaharu Tsuji. Preparation of silver nanoparticles by laser ablation in polyvinylpyrrolidone solutions. Applied Surface Science, 254:5224 5230, 2008.

[85] Tetsuo Sakka, Shuji Iwanaga, Yukio H. Ogata, Akira Matsunawa, and Tadashi Takemoto. Laser ablation at solid-liquid interfaces: An approach from optical emission spectra. The Journal of Chemical Physics, 112:8645-8653, 2000.

[86] A. De Giacomo, M. Dell'Aglio, O. De Pascale, and M. Capitelli. From single pulse to double pulse ns-laser induced breakdown spectroscopy under water: Elemental analysis of aqueous solutions and submerged solid samples. Spectrochimica Acta Part B: Atomic Spectroscopy, 62:721 - 738, 2007.

[87] Takeshi Tsuji, Yasuyuki Tsuboi, Noboru Kitamura, and Masaharu Tsuji. Microsecond-resolved imaging of laser ablation at solid-liquid interface: investigation of formation process of nano-size metal colloids. Applied Surface Science, 229:365 - 371, 2004.

[88] William T. Nichols, Takeshi Sasaki, and Naoto Koshizaki. Laser ablation of a platinum target in water. ii. ablation rate and nanoparticle size distributions. Journal of Applied Physics, 100, 2006.

[89] Vincenzo Amendola and Moreno Meneghetti. Laser ablation synthesis in solution and size manipulation of noble metal nanoparticles. Phys. Chem. Chem. Phys., 11:3805-3821, 2009.

[90] Fumitaka Mafune, Jun ya Kohno, Yoshihiro Takeda, Tamotsu Kondow, and Hisahiro Sawabe. Formation of gold nanoparticles by laser ablation in aqueous solution of surfactant. The Journal of Physical Chemistry B, 105(22):5114-5120, 2001.

[91] Leonid V. Zhigilei, Zhibin Lin, and Dmitriy S. Ivanov. Atomistic modeling of short pulse laser ablation of metals: Connections between melting, spallation, 
and phase explosion. The Journal of Physical Chemistry C, 113:11892-11906, 2009.

[92] J. H. Yoo, S. H. Jeong, X. L. Mao, R. Greif, and R. E. Russo. Evidence for phase-explosion and generation of large particles during high power nanosecond laser ablation of silicon. Applied Physics Letters, 76:783-785, 2000.

[93] William T. Nichols, Takeshi Sasaki, and Naoto Koshizaki. Laser ablation of a platinum target in water. ii. ablation rate and nanoparticle size distributions. Journal of Applied Physics, 100, 2006.

[94] Csaba Laszlo Sajti, Ramin Sattari, Boris N. Chichkov, and Stephan Barcikowski. Gram scale synthesis of pure ceramic nanoparticles by laser ablation in liquid. The Journal of Physical Chemistry C, 114:2421-2427, 2010.

[95] Dmitry G. Shchukin and Helmuth Mohwald. Sonochemical nanosynthesis at the engineered interface of a cavitation microbubble. Phys. Chem. Chem. Phys., 8:3496-3506, 2006.

[96] Tatiana E. Itina. On nanoparticle formation by laser ablation in liquids. The Journal of Physical Chemistry C, 115:5044-5048, 2011.

[97] Chun He, Takeshi Sasaki, Hiroyuki Usui, Yoshiki Shimizu, and Naoto Koshizaki. Fabrication of zno nanoparticles by pulsed laser ablation in aqueous media and ph-dependent particle size: An approach to study the mechanism of enhanced green photoluminescence. Journal of Photochemistry and Photobiology A: Chemistry, 191:66 - 73, 2007.

[98] R. Battino, T. Rettich, and T. Tominaga. The solubility of oxygen and ozone in liquids. Journal of physical and chemical reference data, 12:163-178, 1983.

[99] Shyjumon Ibrahimkutty, Philipp Wagener, Tomy dos Santos Rolo, Dmitry Karpov, Andreas Menzel, Tilo Baumbach, Stephan Barcikowski, and Anton Plech. A hierarchical view on material formation during pulsed-laser synthesis of nanoparticles in liquid. Scientific Reports, 5:16313, 2015.

[100] Daniel Werner, Shuichi Hashimoto, Takuro Tomita, Shigeki Matsuo, and Yoji Makita. Examination of silver nanoparticle fabrication by pulsed-laser ablation of flakes in primary alcohols. The Journal of Physical Chemistry C, 112:13211329, 2008. 
[101] Vincenzo Amendola, Stefano Polizzi, and Moreno Meneghetti. Laser ablation synthesis of gold nanoparticles in organic solvents. The Journal of Physical Chemistry B, 110:7232-7237, 2006.

[102] G. Compagnini, A. A. Scalisi, and O. Puglisi. Production of gold nanoparticles by laser ablation in liquid alkanes. Journal of Applied Physics, 94:7874-7877, 2003.

[103] Fumitaka Mafune, Jun ya Kohno, Yoshihiro Takeda, Tamotsu Kondow, and Hisahiro Sawabe. Formation and size control of silver nanoparticles by laser ablation in aqueous solution. The Journal of Physical Chemistry B, 104:91119117, 2000.

[104] K. Siskova, B. Vlckova, P. Y. Turpin, and C. Fayet. Ion-specific effects on laser ablation of silver in aqueous electrolyte solutions. The Journal of Physical Chemistry C, 112:4435-4443, 2008.

[105] J. P. Colombier, P. Combis, A. Rosenfeld, I. V. Hertel, E. Audouard, and R. Stoian. Optimized energy coupling at ultrafast laser-irradiated metal surfaces by tailoring intensity envelopes: Consequences for material removal from al samples. Phys. Rev. B, 74:224106, 2006.

[106] Christopher Tabor, Wei Qian, , and Mostafa A. El-Sayed. Dependence of the threshold energy of femtosecond laser ejection of gold nanoprisms from quartz substrates on the nanoparticle environment. The Journal of Physical Chemistry C, 111:8934-8941, 2007.

[107] Arun K. Upadhyay, Nail A. Inogamov, Barbel Rethfeld, and Herbert M. Urbassek. Ablation by ultrashort laser pulses: Atomistic and thermodynamic analysis of the processes at the ablation threshold. Phys. Rev. B, 78:045437, 2008 .

[108] D. Zand, P. Nachev, R. Rosenfeld, P. Wagener, and A. Pich. Nanocomposite fibre fabrication via in situ monomer grafting and bonding on laser-generated nanoparticles. Journal of laser micro nanoengineering, 7:21-27, 2012.

[109] Yoshihiro Takeda, Fumitaka Mafuné, and Tamotsu Kondow. Selective degradation of proteins by laser irradiation onto gold nanoparticles in solution. The Journal of Physical Chemistry C, 113:5027-5030, 2009. 
[110] Francesco Giammanco, Emilia Giorgetti, Paolo Marsili, and Anna Giusti. Experimental and theoretical analysis of photofragmentation of au nanoparticles by picosecond laser radiation. The Journal of Physical Chemistry C, 114:33543363, 2010.

[111] Alexander Pyatenko, Munehiro Yamaguchi, and Masaaki Suzuki. Mechanisms of size reduction of colloidal silver and gold nanoparticles irradiated by nd:yag laser. The Journal of Physical Chemistry C, 113:9078-9085, 2009.

[112] Akinori Takami, Hideaki Kurita, , and Seiichiro Koda. Laser-induced size reduction of noble metal particles. The Journal of Physical Chemistry B, 103:12261232, 1999.

[113] Peter Zijlstra, James W. M. Chon, and Min Gu. White light scattering spectroscopy and electron microscopy of laser induced melting in single gold nanorods. Phys. Chem. Chem. Phys., 11:5915-5921, 2009.

[114] Daniel Werner, Shuichi Hashimoto, and Takayuki Uwada. Remarkable photothermal effect of interband excitation on nanosecond laser-induced reshaping and size reduction of pseudospherical gold nanoparticles in aqueous solution. Langmuir, 26:9956-9963, 2010.

[115] S. Link and M. A. El-Sayed. Spectroscopic determination of the melting energy of a gold nanorod. The Journal of Chemical Physics, 114:2362-2368, 2001.

[116] Gregory V. Hartland. Optical studies of dynamics in noble metal nanostructures. Chemical Reviews, 111:3858-3887, 2011.

[117] A.T. Izgaliev, A.V. Simakin, G.A. Shafeev, and F. Bozon-Verduraz. Intermediate phase upon alloying au-ag nanoparticles under laser exposure of the mixture of individual colloids. Chemical Physics Letters, 390:467 - 471, 2004.

[118] Hitomi Muto, Ken Miyajima, , and Fumitaka Mafune. Mechanism of laserinduced size reduction of gold nanoparticles as studied by single and double laser pulse excitation. The Journal of Physical Chemistry C, 112:5810-5815, 2008 .

[119] Renat R. Letfullin, Thomas F. George, and Vladimir P. Zharov. Laser-induced explosion of gold nanoparticles: potential role for nanophotothermolysis of cancer. Nanomedicine, 1:473-80, 2006. 
[120] G.W. Yang. Laser ablation in liquids: Applications in the synthesis of nanocrystals. Progress in Materials Science, 52:648 - 698, 2007.

[121] Anton Plech, Vassilios Kotaidis, Maciej Lorenc, and Johannes Boneberg. Femtosecond laser near-field ablation from gold nanoparticles. Nature Physics, 2:44$47,2006$.

[122] S. Link, C. Burda, M. B. Mohamed, B. Nikoobakht, , and M. A. El-Sayed. Laser photothermal melting and fragmentation of gold nanorods: Energy and laser pulse-width dependence. The Journal of Physical Chemistry A, 103:1165-1170, 1999.

[123] Vassilios Kotaidis and Anton Plech. Cavitation dynamics on the nanoscale. Applied Physics Letters, 87, 2005.

[124] V. Kotaidis, C. Dahmen, G. von Plessen, F. Springer, and A. Plech. Excitation of nanoscale vapor bubbles at the surface of gold nanoparticles in water. The Journal of Chemical Physics, 124, 2006.

[125] Daniel Werner and Shuichi Hashimoto. Improved working model for interpreting the excitation wavelength- and fluence-dependent response in pulsed laserinduced size reduction of aqueous gold nanoparticles. The Journal of Physical Chemistry C, 115:5063-5072, 2011.

[126] J. B. Pendry, A. Aubry, D. R. Smith, and S. A. Maier. Transformation optics and subwavelength control of light. Science, 337(6094):549-552, 2012.

[127] Beatriz Roldan Cuenya. Synthesis and catalytic properties of metal nanoparticles: Size, shape, support, composition, and oxidation state effects. Thin Solid Films, 518(12):3127 - 3150, 2010.

[128] William H. Zachariasen. Theory of x-ray diffraction in crystals. Dover, USA, 2004.

[129] C. J. Humphreys. The significance of Bragg's law in electron diffraction and microscopy, and Bragg's second law. Acta Crystallographica Section A, 69(1):4550, 2013.

[130] C.T. Sah. Fundamentals of Solid-state Electronics. World Scientific, 1991. 
[131] D.B. Williams and C.B. Carter. Transmission Electron Microscopy: A Textbook for Materials Science. Number v. 2 in Cambridge library collection. Springer, 2009 .

[132] S. Horiuchi. Fundamentals of high-resolution transmission electron microscopy. North-Holland, 1994.

[133] B.K. Vainshtein. Fundamentals of Crystals: Symmetry, and Methods of Structural Crystallography. Modern crystallography. Springer Berlin Heidelberg, 2013.

[134] DC Bell and AJ Garratt-Reed. Energy Dispersive X-ray Analysis in the Electron Microscope. Microscopy handbooks. Taylor and Francis, 2003.

[135] V.I. Gavrilenko. Optics of Nanomaterials. Pan Stanford, 2011.

[136] Jie Cao, Tong Sun, and Kenneth T.V. Grattan. Gold nanorod-based localized surface plasmon resonance biosensors: A review. Sensors and Actuators B: Chemical, 195:332 - 351, 2014.

[137] Surbhi Lal, Stephan Link, and Naomi J. Halas. Nano-optics from sensing to waveguiding. Nature photonics, 1:641 - 648, 2007.

[138] Robert A Taylor, Patrick E Phelan, Todd P Otanicar, Ronald Adrian, and Ravi Prasher. Nanofluid optical property characterization: towards efficient direct absorption solar collectors. Nanoscale research letters, 6:225, 2011.

[139] Paul Mulvaney. Surface plasmon spectroscopy of nanosized metal particles. Langmuir, 12(3):788-800, 1996.

[140] Stephan Link, , and Mostafa A. El-Sayed. Spectral properties and relaxation dynamics of surface plasmon electronic oscillations in gold and silver nanodots and nanorods. The Journal of Physical Chemistry B, 103:8410-8426, 1999.

[141] F F Fankhauser. Application of static and dynamic light scattering-a review. KLINISCHE MONATSBLATTER FUR AUGENHEILKUNDE, 227:194-198, 2010.

[142] B.J. Berne and R. Pecora. Dynamic Light Scattering: With Applications to Chemistry, Biology, and Physics. Dover Books on Physics. Dover Publications, 2013 . 
[143] D Quemada and C Beril. Energy of interaction in colloids and its implications in rheological modeling. Advances in Colloid and Interface Science, 98:51-85, 2002 .

[144] A.R. Boccaccini, O. van der Biest, J.B. Talbot, Electrochemical Society. Electrodeposition Division, and Engineering Foundation (U.S.). Electrophoretic Deposition, Fundamentals and Applications: Proceedings of the International Symposium. Proceedings (Electrochemical Society). Electrochemical Society, 2002 .

[145] N.A. Gokcen and R.G. Reddy. Thermodynamics. Language of science. Springer US, 2013.

[146] S U S Choi. Enhancing thermal conductivity of fluids with nanoparticles. Proceddings of the 1995 ASME International Mechanical Engenieering Congress and Exposition (USA), page 99, 1995.

[147] C.J. Cremers, H.A. Fine, and H.A. Fine. Thermal Conductivity. Thermal conductivity. Springer, 1991.

[148] M.S. Lee. Mass Spectrometry Handbook. Wiley Series on Pharmaceutical Science and Biotechnology: Practices, Applications and Methods. Wiley, 2012.

[149] Carsten Momma, Stefan Nolte, Boris N Chichkov, Ferdinand von Alvensleben, and Andreas Tunnermann. Precise laser ablation with ultrashort pulses. Appl. Surface Science, 109:15 - 19, 1997.

[150] Gerrard A. and Burch J. M. Introduction to Matrix Methods in Optics. Dover, USA, 1994.

[151] A. Couairon and A. Mysyrowicz. Femtosecond filamentation in transparent media. Physics Reports, 441(2-4):47 - 189, 2007.

[152] Ana Menendez-Manjon, Philipp Wagener, and Stephan Barcikowski. Transfermatrix method for efficient ablation by pulsed laser ablation and nanoparticle generation in liquids. The Journal of Physical Chemistry C, 115:5108-5114, 2011.

[153] P. E. Glaser. Power from the sun: its future. Science, 162:857-861, 1968. 
[154] Bharath Dudda and Donghyun Shin. Effect of nanoparticle dispersion on specific heat capacity of a binary nitrate salt eutectic for concentrated solar power applications. International Journal of Thermal Sciences, 69:37 - 42, 2013.

[155] Xiaobo Chen, Can Li, Michael Gratzel, Robert Kostecki, and Samuel S. Mao. Nanomaterials for renewable energy production and storage. Chem. Soc. Rev., 41:7909-7937, 2012.

[156] James Clerk Maxwell. A Treatise on Electricity and Magnetism. Clarendon Press, Oxford, UK, 1873.

[157] Das S. K., Choi S. U. S., Yu W., and Pradeep T. Nanofluids. Science and Technology. John Wiley and Sons, Inc., USA, 2008.

[158] Ravikanth S. Vajjha and Debendra K. Das. A review and analysis on influence of temperature and concentration of nanofluids on thermophysical properties, heat transfer and pumping power. International Journal of Heat and Mass Transfer, 55:4063 - 4078, 2012.

[159] Wenhua Yu, David M. France, Elena V. Timofeeva, Dileep Singh, and Jules L. Routbort. Comparative review of turbulent heat transfer of nanofluids. International Journal of Heat and Mass Transfer, 55:5380 - 5396, 2012.

[160] B.H. Salman, H.A. Mohammed, K.M. Munisamy, and A. Sh. Kherbeet. Characteristics of heat transfer and fluid flow in microtube and microchannel using conventional fluids and nanofluids: A review. Renewable and Sustainable Energy Reviews, 28:848 - 880, 2013.

[161] Adnan M. Hussein, K.V. Sharma, R.A. Bakar, and K. Kadirgama. A review of forced convection heat transfer enhancement and hydrodynamic characteristics of a nanofluid. Renewable and Sustainable Energy Reviews, 29:734 - 743, 2014.

[162] J. A. Eastman, S. U. S. Choi, S. Li, W. Yu, and L. J. Thompson. Anomalously increased effective thermal conductivities of ethylene glycol-based nanofluids containing copper nanoparticles. Applied Physics Letters, 78(6):718-720, 2001.

[163] Godson L., Raja B., Mohan Lal D., and Wongwises S. Experimental investigation on the thermal conductivity and viscosity of silver-deionized water nanofluid. Experimental heat transfer, 23:317 - 332, 2010. 
[164] Seung Won Lee, Sung Dae Park, Sarah Kang, In Cheol Bang, and Ji Hyun Kim. Investigation of viscosity and thermal conductivity of sic nanofluids for heat transfer applications. International Journal of Heat and Mass Transfer, $54: 433-438,2011$.

[165] Huaqing Xie, Jinchang Wang, Tonggeng Xi, Yan Liu, Fei Ai, and Qingren Wu. Thermal conductivity enhancement of suspensions containing nanosized alumina particles. Journal of Applied Physics, 91(7):4568-4572, 2002.

[166] S. U. S. Choi, Z. G. Zhang, W. Yu, F. E. Lockwood, and E. A. Grulke. Anomalous thermal conductivity enhancement in nanotube suspensions. Applied Physics Letters, 79(14):2252-2254, 2001.

[167] E. Sani, S. Barison, C. Pagura, L. Mercatelli, P. Sansoni, D. Fontani, D. Jafrancesco, and F. Francini. Carbon nanohorns-based nanofluids as direct sunlight absorbers. Opt. Express, 18(5):5179-5187, 2010.

[168] Lifei Chen, Wei Yu, and Huaqing Xie. Enhanced thermal conductivity of nanofluids containing ag/mwnt composites. Powder Technology, 231:18 - 20, 2012 .

[169] Donghyun Shin and Debjyoti Banerjee. Enhanced specific heat capacity of nanomaterials synthesized by dispersing silica nanoparticles in eutectic mixtures. Journal of Heat Transfer, 135:032801, 2011.

[170] J. E. Minardi and H. N. Chuang. Performance of a "black" liquid flat-plate solar collector. Solar Energy, 17:179 - 183, 1975.

[171] Prashant K. Jain, Xiaohua Huang, Ivan H. El-Sayed, and Mostafa A. El-Sayed. Review of some interesting surface plasmon resonance-enhanced properties of noble metal nanoparticles and their applications to biosystems. Plasmonics, 2:107-118, 2007.

[172] Neumann Oara, Neumann Albert D., Silva Edgar, Ayala-Orozco Ciceron, Tian Shu, Nordlander Peter, and Halas Naomi J. Nanoparticle-mediated, lightinduced phase separations. Nano Letters, 15:7880-7885, 2015.

[173] Todd P. Otanicar, Patrick E. Phelan, Ravi S. Prasher, Gary Rosengarten, and Robert A. Taylor. Nanofluid-based direct absorption solar collector. Journal of Renewable and Sustainable Energy, 2, 2010. 
[174] Moradi A., Sani E., Simonetti M., Francini F., Chiavazzo E., and Asinari P. Carbon-nanohorn based nanofluids for a direct absorption solar collector for civil application. Journal of nanoscience and nanotechnology, 15:3488-3495, 2015 .

[175] Andrej Lenert and Evelyn N Wang. Optimization of nanofluid volumetric receivers for solar thermal energy conversion. Solar Energy, 86:253-265, 2012.

[176] Zhongyang Luo, Cheng Wang, Wei Wei, Gang Xiao, and Mingjiang Ni. Performance improvement of a nanofluid solar collector based on direct absorption collection (dac) concepts. International Journal of Heat and Mass Transfer, $75: 262-271,2014$.

[177] Hogan Nathaniel J., Urban Alexander S., Ayala-Orozco Ciceron, Pimpinelli Alberto, Nordlander Peter, and Halas Naomi J. Nanoparticles heat through light localization. Nano Letters, 14:4640-4645, 2014.

[178] Neumann Oara, Urban Alexander S., Day Jared, Lal Surbhi, Nordlander Peter, and Halas Naomi J. Solar vapor generation enabled by nanoparticles. ACS Nano, 7:42-49, 2013.

[179] R. Saidur, T.C. Meng, Z. Said, M. Hasanuzzaman, and A. Kamyar. Evaluation of the effect of nanofluid-based absorbers on direct solar collector. International Journal of Heat and Mass Transfer, 55:5899 - 5907, 2012.

[180] Yuki Kameya and Katsunori Hanamura. Enhancement of solar radiation absorption using nanoparticle suspension. Solar Energy, 85(2):299 - 307, 2011.

[181] E. Sani, L. Mercatelli, S. Barison, C. Pagura, F. Agresti, L. Colla, and P. Sansoni. Potential of carbon nanohorn-based suspensions for solar thermal collectors. Solar Energy Materials and Solar Cells, 95(11):2994 - 3000, 2011.

[182] Sani E., Di Ninni P., Colla L., Barison S., and Agresti F. Optical properties of mixed nanofluids containing carbon nanohorns and silver nanoparticles for solar energy applications. Journal of nanoscience and nanotechnology, 15:3568 $-3573,2015$.

[183] Prasher Ravi, Phelan Patrick E., and Bhattacharya Prajesh. Effect of aggregation kinetics on the thermal conductivity of nanoscale colloidal solutions (nanofluid). Nano Letters, 6(7):1529-1534, 2006. 
[184] J. W. Gao, R. T. Zheng, H. Ohtani, D. S. Zhu, and G. Chen. Experimental investigation of heat conduction mechanisms in nanofluids. clue on clustering. Nano Letters, 9:4128-4132, 2009.

[185] Paul Anastas and John Warner. 12 principles of green chemistry. urlhttp://www.acs.org/content/acs/en/greenchemistry/what-is-greenchemistry/principles/12-principles-of-green-chemistry.html, 1998.

[186] Alexander Pyatenko, Hongqiang Wang, Naoto Koshizaki, and Takeshi Tsuji. Mechanism of pulse laser interaction with colloidal nanoparticles. Laser and Photonics Reviews, 7:596-604, 2013.

[187] S. Besner, A.V. Kabashin, and M. Meunier. Two-step femtosecond laser ablation-based method for the synthesis of stable and ultra-pure gold nanoparticles in water. Applied Physics A, 88(2):269-272, 2007.

[188] F. A. Videla, G. A. Torchia, D. C. Schinca, L. B. Scaffardi, P. Moreno, C. Mendez, L. J. Giovanetti, J. M. Ramallo Lopez, and L. Roso. Analysis of the main optical mechanisms responsible for fragmentation of gold nanoparticles by femtosecond laser radiation. Journal of Applied Physics, 107, 2010.

[189] Jean-Philippe Sylvestre, Andrei V. Kabashin, Edward Sacher, Michel Meunier, and John H. T. Luong. Stabilization and size control of gold nanoparticles during laser ablation in aqueous cyclodextrins. Journal of the American Chemical Society, 126:7176-7177, 2004.

[190] Muto Hitomi, Yamada Kunihiro, Miyajima Ken, and Mafune Fumitaka. Estimation of surface oxide on surfactant-free gold nanoparticles laser-ablated in water. The Journal of Physical Chemistry C, 111:17221-17226, 2007.

[191] A. Hahn, S. Barcikowski, and B. N. Chichkov. Influences on nanoparticle production during pulsed laser ablation. Journal of laser micro nanoengineering, 3:73-77, 2008.

[192] F. Hajiesmaeilbaigi, A. Mohammadalipour, J. Sabbaghzadeh, S. Hoseinkhani, and H. R. Fallah. Preparation of silver nanoparticles by laser ablation and fragmentation in pure water. Laser Physics Letters, 3:252-256, 2006.

[193] Ferreira P. H. D., Vivas M. G., Boni L. De, dosSantos Jr. D. S., Balogh D. T., Misoguti L., and Mendonca C. R. Femtosecond laser induced synthesis of au nanoparticles mediated by chitosan. Optics Express, 20(1):136-142, 2008. 
[194] Behzad Tangeysh, Katharine Moore Tibbetts, Johanan H. Odhner, Bradford B. Wayland, and Robert J. Levis. Gold nanoparticle synthesis using spatially and temporally shaped femtosecond laser pulses: Post-irradiation auto-reduction of aqueous [aucl4] $]^{-}$. The Journal of Physical Chemistry C, 117:18719-18727, 2013.

[195] Xiong Liu, Mark Atwater, Jinhai Wang, and Qun Huo. Extinction coefficient of gold nanoparticles with different sizes and different capping ligands. Colloids and Surfaces B: Biointerfaces, 58(1):3 - 7, 2007.

[196] Boopathi S., Senthilkumar S., and Phaniet K. L. Facile and one pot synthesis of gold nanoparticles using tetraphenylborate and polyvinylpyrrolidone for selective colorimetric detection of mercury ions in aqueous medium. Analytical Meth. Chem., page 348965, 2012.

[197] Bohren C. F. and Huffman D. R. Absorption and scattering of light by small particles. John Wiley and Sons, Inc., USA, 1983.

[198] Madsen S. J., Patterson M. S., and Wilson B. C. The use of india ink as an optical absorber in tissue-simulating phantoms. Physics in medicine and biology, 37:985 - 993, 1992.

[199] George M. Hale and Marvin R. Querry. Optical constants of water in the 200-nm to 200- $\mu \mathrm{m}$ wavelength region. Appl. Opt., 12(3):555-563, 1973.

[200] Rakic A. D., Djurisic A. B., Elazar J. M., and Majewski M. L. Optical properties of metallic films for vertical-cavity optoelectronic devices. Appl. Opt., 37:5271$5283,1998$.

[201] CIE Technical Report no. 85. Solar spectral irradiance, 1989.

[202] Visinee Trisaksri and Somchai Wongwises. Critical review of heat transfer characteristics of nanofluids. Renewable and Sustainable Energy Reviews, 11(3):512 $-523,2007$.

[203] Zhou Mingzheng, Xia Guodong, Li Jian, Chai Lei, and Zhou Lijun. Analysis of factors influencing thermal conductivity and viscosity in different kinds of surfactant solutions. Experimental Thermal and Fluid Science, 36:22 - 29, 2012.

[204] C.J. Winter, R.L. Sizmann, and L.L. Vant-Hull. Solar Power Plants: Fundamentals, Technology, Systems, Economics. Springer Berlin Heidelberg, 2011. 
[205] Robert A Taylor, Todd P Otanicar, Yasitha Herukerrupu, Fabienne Bremond, Gary Rosengarten, Evatt R Hawkes, Xuchuan Jiang, and Sylvain Coulombe. Feasibility of nanofluid-based optical filters. Applied Optics, 52:1413-22, 2013.

[206] Long Zhang, Jian Liu, Guodong He, Zhuocheng Ye, Xiaoming Fang, and Zhengguo Zhang. Radiative properties of ionic liquid-based nanofluids for mediumto-high-temperature direct absorption solar collectors. Solar Energy Materials and Solar Cells, 130:521-528, 2014.

[207] M Karami, M A Akhavan Bahabadi, S Delfani, and A Ghozatloo. A new application of carbon nanotubes nanofluid as working fluid of low-temperature direct absorption solar collector. Solar Energy Materials and Solar Cells, 121:114-118, 2014 .

[208] C. Wang, J. Yang, and Y. Ding. Phase transfer based synthesis and thermophysical properties of au/therminol vp-1 nanofluids. Progress in natural science, 23:338-342, 2013.

[209] Yoojung Kwon, MG Kim, Y Kim, Y Lee, and JP Cho. Effect of capping agents in tin nanoparticles on electrochemical cycling. Electrochemical and solid-state letters, 9:A34-A38, 2006.

[210] G. Krishna Podagatlapalli, Syed Hamad, Surya P. Tewari, S. Sreedhar, Muvva D. Prasad, and S. Venugopal Rao. Silver nano-entities through ultrafast double ablation in aqueous media for surface enhanced raman scattering and photonics applications. Journal of Applied Physics, 113, 2013.

[211] Cheng-An J. Lin, Ting-Ya Yang, Chi-Hsien Lee, Sherry H. Huang, Ralph A. Sperling, Marco Zanella, Jimmy K. Li, Ji-Lin Shen, Hsueh-Hsiao Wang, Hung-I Yeh, Wolfgang J. Parak, and Walter H. Chang. Synthesis, characterization, and bioconjugation of fluorescent gold nanoclusters toward biological labeling applications. ACS Nano, 3(2):395-401, 2009.

[212] Sreeram Cingarapu, Dileep Singh, Elena V. Timofeeva, and Michael R. Moravek. Nanofluids with encapsulated tin nanoparticles for advanced heat transfer and thermal energy storage. International Journal of Energy Research, 38:51-59, 2013. 
[213] Stephan Barcikowski and Giuseppe Compagnini. Advanced nanoparticle generation and excitation by lasers in liquids. Phys. Chem. Chem. Phys., 15:30223026, 2013.

[214] Wei-Er Lu, Mei-Ling Zheng, Wei-Qiang Chen, Zhen-Sheng Zhao, and XuanMing Duan. Gold nanoparticles prepared by glycinate ionic liquid assisted multi-photon photoreduction. Phys. Chem. Chem. Phys., 14:11930-11936, 2012.

[215] Sambandam Anandan and Jerry J Wu. Sonochemical synthesis of carbon supported Sn nanoparticles and its electrochemical application. Ultrasonics Sonochemistry, 21:1954-1957, 2014.

[216] Gnana S Balasubramaniam, Abdullah M Asiri, Abdullah H Qusti, Jerry J Wu, and Sambandam Anandan. Sonochemically synthesized $\mathrm{MnO} 2$ nanoparticles as electrode material for supercapacitors. Ultrasonics Sonochemistry, 21:19331938, 2014.

[217] Naoki Shirai, Satoshi Uchida, and Fumiyoshi Tochikubo. Synthesis of metal nanoparticles by dual plasma electrolysis using atmospheric dc glow discharge in contact with liquid. Japanese Journal of Applied Physics, 53:046202, 2014.

[218] D. J. Shaw. Introduction to colloid and surface chemistry. ButterworthHeinemann, UK, 1991.

[219] H. E. Bergna and W. O. Roberts. Colloidal silica: Fundamentals and applications. CRC Taylor and Francis, USA, 2005.

[220] Hua Bai, Chun Li, and Gaoquan Shi. Functional composite materials based on chemically converted graphene. Advanced Materials, 23:1089-1115, 2011.

[221] Hummers Jr. William S. and Offeman Richard E. Preparation of graphitic oxide. Journal of the American Chemical Society, 80:1339-1339, 1958.

[222] Yuan Zhao and Yanwu Zhu. Graphene-based hybrid films for plasmonic sensing. Nanoscale, 7:14561-14576, 2015.

[223] Junwu Zhu, Guiyu Zeng, Fude Nie, Xiaoming Xu, Sheng Chen, Qiaofeng Han, and Xin Wang. Decorating graphene oxide with cuo nanoparticles in a waterisopropanol system. Nanoscale, 2:988-994, 2010. 
[224] Yury V. Stebunov, Olga A. Aftenieva, Aleksey V. Arsenin, and Valentyn S. Volkov. Highly sensitive and selective sensor chips with graphene-oxide linking layer. ACS Applied Materials and Interfaces, 7:21727-21734, 2015.

[225] Fang Wen, Yanhua Dong, Lu Feng, Song Wang, Sichun Zhang, and Xinrong Zhang. Horseradish peroxidase functionalized fluorescent gold nanoclusters for hydrogen peroxide sensing. Analytical Chemistry, 83:1193-1196, 2011.

[226] Li Shang, Shaojun Dong, and G. Ulrich Nienhaus. Ultra-small fluorescent metal nanoclusters: Synthesis and biological applications. Nano Today, 6:401-418, 2011.

[227] Cheng-An J. Lin, Chih-Hsien Lee, Jyun-Tai Hsieh, Hsueh-Hsiao Wang, and Jimmy K. Li. Synthesis of fluorescent metallic nanoclusters toward biomedical application: Recent progress and present challenges. Journal of medical and biological engineering, 29:276 - 283, 2009.

[228] Cheng-An J. Lin, Ting-Ya Yang, Chih-Hsien Lee, Sherry H. Huang, Ralph A. Sperling, Marco Zanella, Jimmy K. Li, Ji-Lin Shen, Hsueh-Hsiao Wang, Hung-I Yeh, Wolfgang J. Parak, and Walter H. Chang. Synthesis, characterization, and bioconjugation of fluorescent gold nanoclusters toward biological labeling applications. ACS Nano, 3:395-401, 2009.

[229] Primo Ana, Esteve-Adell Ivan, F. Blandez Juan, Dhakshinamoorthy Amarajothi, Alvaro Mercedes, Candu Natalia, Coman Simona M., Parvulescu Vasile I., and Garcia Hermenegildo. High catalytic activity of oriented 2.0.0 copper(i) oxide grown on graphene film. Nature Communications, 6:8561, 2015.

[230] Masatake Haruta. Size- and support-dependency in the catalysis of gold. Catalysis Today, 36:153 - 166, 1997.

[231] Yin Bingsheng, Ma Houyi, Wang Shuyun, and Chen Shenhao. Electrochemical synthesis of silver nanoparticles under protection of poly(n-vinylpyrrolidone). The Journal of Physical Chemistry B, 107:8898-8904, 2003.

[232] Ling Zhang, Wenxin Niu, and Guobao Xu. Synthesis and applications of noble metal nanocrystals with high-energy facets. Nano Today, 7:586 - 605, 2012.

[233] Zhihong Tang, Shuling Shen, Jing Zhuang, and Xun Wang. Noble-metalpromoted three-dimensional macroassembly of single-layered graphene oxide. Angewandte Chemie International Edition, 49:4603-4607, 2010. 
[234] Jie Zheng, Philip R. Nicovich, and Robert M. Dickson. Highly fluorescent noble-metal quantum dots. Annual Review of Physical Chemistry, 58:409-431, 2007.

[235] H Qian, M Zhu, Z Wu, and R. Jin. Quantum sized gold nanoclusters with atomic precision. Acc. Chem. Res., 45:1470, 2012.

[236] Fumitaka Mafune, Jun ya Kohno, Yoshihiro Takeda, and Tamotsu Kondow. Growth of gold clusters into nanoparticles in a solution following laser-induced fragmentation. The Journal of Physical Chemistry B, 106:8555-8561, 2002.

[237] Vincenzo Amendola and Moreno Meneghetti. What controls the composition and the structure of nanomaterials generated by laser ablation in liquid solution? Phys. Chem. Chem. Phys., 15:3027-3046, 2013.

[238] Rafael R. Gattass and Eric Mazur. Femtosecond laser micromachining in transparent materials. Nature photonics, 2:219-225, 2008.

[239] A. Barchanski, N. Hashimoto, S. Petersen, C. L. Sajti, and S. Barcikowski. Impact of spacer and strand length on oligonucleotide conjugation to the surface of ligand-free laser-generated gold nanoparticles. Bioconjugate Chemistry, 23:908-915, 2012.

[240] AM Morales and CM Lieber. A laser ablation method for the synthesis of crystalline semiconductor nanowires. Science, 279:208-211, 1998.

[241] Ibrahimkutty Shyjumon, Wagener Philipp, dos Santos Rolo Tomy, Karpov Dmitry, Menzel Andreas, Baumbach Tilo, Barcikowski Stephan, and Plech Anton. A hierarchical view on material formation during pulsed-laser synthesis of nanoparticles in liquid. Scientific Reports, 5:16313, 2015.

[242] Xi Yang, Mingsheng Xu, Weiming Qiu, Xiaoqiang Chen, Meng Deng, Jinglin Zhang, Hideo Iwai, Eiichiro Watanabe, and Hongzheng Chen. Graphene uniformly decorated with gold nanodots: in situ synthesis, enhanced dispersibility and applications. J. Mater. Chem., 21:8096-8103, 2011.

[243] Toby Sainsbury, Jacek Stolarczyk, , and Donald Fitzmaurice. An experimental and theoretical study of the self-assembly of gold nanoparticles at the surface of functionalized multiwalled carbon nanotubes. The Journal of Physical Chemistry B, 109(34):16310-16325, 2005. 
[244] Andreas Hirsch, Jan M. Englert, and Frank Hauke. Wet chemical functionalization of graphene. Accounts of Chemical Research, 46:87-96, 2013.

[245] Marcus Lau, Ina Haxhiaj, Philipp Wagener, Romuald Intartaglia, Fernando Brandi, Junji Nakamura, and Stephan Barcikowski. Ligand-free gold atom clusters adsorbed on graphene nano sheets generated by oxidative laser fragmentation in water. Chemical Physics Letters, 610-611:256 - 260, 2014.

[246] Daniel R. Dreyer, Rodney S. Ruoff, and Christopher W. Bielawski. From conception to realization: An historial account of graphene and some perspectives for its future. Angewandte Chemie International Edition, 49:9336-9344, 2010.

[247] I.T. BELASH, O.V. ZHARIKOV, and A.V. PALNICHENKO. Superconductivity of gic with li, na and k. Synthetic metals, 34:455-460, 1990.

[248] Sasha Stankovich, Dmitriy A. Dikin, Richard D. Piner, Kevin A. Kohlhaas, Alfred Kleinhammes, Yuanyuan Jia, Yue Wu, SonBinh T. Nguyen, and Rodney S. Ruoff. Synthesis of graphene-based nanosheets via chemical reduction of exfoliated graphite oxide. Carbon, 45:1558 - 1565, 2007.

[249] Daniel R. Dreyer, Sungjin Park, Christopher W. Bielawski, and Rodney S. Ruoff. The chemistry of graphene oxide. Chem. Soc. Rev., 39:228-240, 2010. 


\section{Scientific production}

\section{Published papers}

- Torres-Mendieta R., Mondragón R., Juliá E., Mendoza-Yero O., Cordoncillo E., Lancis J. and Mínguez-Vega G. 2014 Fabrication of gold nanoparticles in Therminol VP-1 by laser ablation and fragmentation with fs pulses Laser Phys. Lett. 11 pp 126001.

- Torres-Mendieta R., Mondragón R., Juliá E., Mendoza-Yero O., Lancis J. and Mínguez-Vega G. 2015 Fabrication of high stable gold nanofluid by pulsed laser ablation in liquids Adv. Mater. Lett. 6 pp 1037.

\section{Recently submitted papers}

- Torres-Mendieta R., Ventura-Espinosa D., Sabater S., Lancis J., Mínguez-Vega G. and Mata J. 2016 Laser-based method: An ideal tool for gold decoration of graphene sheets Scientific Reports, Submitted paper.

- Mondragón R., Torres-Mendieta R., Meucci M., Mínguez-Vega G., Juliá E. and Sani E. 2016 Synthesis and characterization of gold / water nanofluids suitable for thermal applications produced by Femtosecond Laser Radiation Journal of Photonics for Energy, Submitted paper.

\section{Contributions to international conferences}

- Torres-Mendieta R., Mondragón R., Mendoza-Yero O., MínguezVega G. and Lancis J. 2014 Nanoparticle fabrication in Therminol VP-1 by Pulsed Laser Ablation in Liquids in two steps II International Conference on Applications of Optics and Photonics. (Portugal:Aveiro). 
- Torres-Mendieta R., Mondragón R., Mendoza-Yero O. and Lancis J. 2014 Gold nanoparticle fabrication in Therminol VP-1 by Pulsed Laser Ablation in Liquids 23rd Congress of the International Commission of Optics. (Spain:Santiago de Compostela).

- Torres-Mendieta R., Mondragón R., Andrés P. and Lancis J. 2014 Nanoparticle agglomeration in Therminol VP-1 after being produced by Pulsed Laser Ablation in Liquids in two steps The European Optical Society Annual Meeting. (Germany:Berlin).

- Torres-Mendieta R., Mondragón R., Juliá E., Mendoza-Yero O., Andrés P. and Lancis J. 2015 Enhance Nanofluid Fabricated by Pulse Laser Ablation in Liquid with Application in Solar Power Plants CLEO(R) / Europe - EQEC 2015. (Germany:Munich).

- Torres-Mendieta R., Mondragón R., Juliá E., Mendoza-Yero O., Lancis J. and Mínguez-Vega G. 2015 Improvement of stability properties in heat transfer nanofluids fabricated by Pulsed Laser Ablation in Liquids Open Readings 2015. (Lithuania:Vilnius).

- Torres-Mendieta R., Mondragón R., Juliá E., Mendoza-Yero O., Lancis J. and Mínguez-Vega G. 2015 Fabrication of high stable gold nanofluid by Pulsed Laser Ablation in Liquids Advanced Materials World Congress 2015. (Sweden:Stockholm).

- Torres-Mendieta R., Mendoza-Yero O., Lancis J. and Mínguez-Vega G. 2015 High quality "green" nanoparticles via femtosecond laser ablation in liquids Ultrafast Science and Technology Spain 2015. (Spain:Madrid).

- Torres-Mendieta R., Ventura-Espinosa D., Sabater S., Lancis J., Mata J. and Mínguez-Vega G. 2016 Direct laser synthesis of gold nanoparticles and immobilization on graphene sheets 4th Conference on Advanced Nanoparticle Generation and Excitation by Lasers in Liquids (ANGEL). (Germany:Essen).

- Torres-Mendieta R., Mondragón R., Puerto-Belda V., Mendoza-Yero O., Andrés P., Lancis J., Juliá J. and Mínguez-Vega G. 2016 Solar nanofluid fabricated by a femtosecond laser-assisted technique 4th Conference on Advanced Nanoparticle Generation and Excitation by Lasers in Liquids (ANGEL). (Germany:Essen). 


\section{Contributions to national conferences}

- Torres-Mendieta R., Mondragón R., Doñate-Buendía C., Andrés P., Lancis J. and Mínguez-Vega G. 2015 Laser-assisted technique for the fabrication of an oil-based nanofluid XI Reunión Nacional de Óptica. (Spain:Salamanca).

- Torres-Mendieta R., Puerto-Belda V., Mondragón R., Doñate-Buendía C., Mendoza-Yero O., Lancis J., Juliá J. and Mínguez-Vega G. 2016 Synthesis of a solar tin-based nanofluid by a femtosecond laser-assisted approach XIV Congreso nacional de materiales. (Spain:Gijón). 